\title{
Detection of Latent Heteroscedasticity and Group-Based Regression Effects in Linear Models via Bayesian Model Selection
}

\author{
Thomas Anthony Metzger
}

\author{
Dissertation submitted to the Faculty of the \\ Virginia Polytechnic Institute and State University \\ in partial fulfillment of the requirements for the degree of \\ Doctor of Philosophy \\ in \\ Statistics
}

Christopher T. Franck, Chair

Marco A. R. Ferreira

Robert B. Gramacy

Leanna L. House

July 18, 2019

Blacksburg, Virginia

Keywords: heteroscedasticity, model selection, Bayesian

Copyright 2019, Thomas Anthony Metzger 


\title{
Detection of Latent Heteroscedasticity and Group-Based Regression Effects in Linear Models via Bayesian Model Selection
}

\author{
Thomas Anthony Metzger
}

(ABSTRACT)

Standard linear modeling approaches make potentially simplistic assumptions regarding the structure of categorical effects that may obfuscate more complex relationships governing data. For example, recent work focused on the two-way unreplicated layout has shown that hidden groupings among the levels of one categorical predictor frequently interact with the ungrouped factor. We extend the notion of a "latent grouping factor" to linear models in general. The proposed work allows researchers to determine whether an apparent grouping of the levels of a categorical predictor reveals a plausible hidden structure given the observed data. Specifically, we offer Bayesian model selection-based approaches to reveal latent groupbased heteroscedasticity, regression effects, and/or interactions. Failure to account for such structures can produce misleading conclusions. Since the presence of latent group structures is frequently unknown a priori to the researcher, we use fractional Bayes factor methods and mixture $g$-priors to overcome lack of prior information. We provide an R package, slgf, that implements our methodology in practice, and demonstrate its usage in practice. 


\title{
Detection of Latent Heteroscedasticity and Group-Based Regression Effects in Linear Models via Bayesian Model Selection
}

\author{
Thomas Anthony Metzger
}

(GENERAL AUDIENCE ABSTRACT)

Statistical models are a powerful tool for describing a broad range of phenomena in our world. However, many common statistical models may make assumptions that are overly simplistic and fail to account for key trends and patterns in data. Specifically, we search for hidden structures formed by partitioning a dataset into two groups. These two groups may have distinct variability, statistical effects, or other hidden effects that are missed by conventional approaches. We illustrate the ability of our method to detect these patterns through a variety of disciplines and data layouts, and provide software for researchers to implement this approach in practice. 


\section{Dedication}

To the many, many educators throughout my life whose knowledge, enthusiasm, and patience have led me to this rich and rewarding field. 


\section{Acknowledgments}

My time at Virginia Tech has been one of the most unexpected and rewarding experiences of my life. I am eternally grateful to the many family members, teachers, colleagues, friends, and students whose support made it possible.

Thank you to my family, for fostering my desire to learn. Your patience and enthusiasm for my education and new career have been unfaltering. I am happy to return to The Great State of Ohio, only six years late.

Thank you the faculty and staff of Hoban High School, who were the first to show me what mathematics and statistics could be. Thank you to my classmates, whose friendships that have remained over the years will always be appreciated.

Thank you to the faculty and staff of The Ohio State University, for your role in my development as a mathematician. I am proud to have the opportunity to return to work with the faculty and students I know well. Thank you also to my friends from Ohio State, who have always stood with me when I needed it.

Thank you to the faculty, staff, and students of Staunton High School, and to my friends there for their support. I will always be grateful for my time and experiences in Staunton that laid the foundation for who I am as an educator.

Thank you to Dr. Jeff Birch, for giving me the opportunity to join Virginia Tech and earn my Ph.D.

Thank you to the faculty and graduate students of the Virginia Tech Department of Statistics. It has been a privilege to learn and work with you for five years, and your friendship has 
been an integral part of my success as a researcher and teacher. I look forward to remaining a part of the Virginia Tech community.

Thank you to Marco Ferreira, Bobby Gramacy, and Leanna House, for your suggestions on this work, and for your mentorship. I am proud to have completed this work with your guidance.

Finally, thank you to Chris Franck, a great statistician, researcher, teacher, and friend. Every accomplishment I might have in my career is thanks to you. I begin my new career with great optimism as a result of your guidance. 


\section{Contents}

1 Introduction 1

2 Suspected Latent Grouping Factor Methodology $\quad 4$

2.1 Introduction . . . . . . . . . . . . . . . . . . . . 4

2.2 Proposed Method . . . . . . . . . . . . . . . . 7

2.2.1 Specification of Linear Models with Categorical Predictors . . . . . . 7

2.2.2 Bayesian Model Selection Details . . . . . . . . . . . . . . . 10

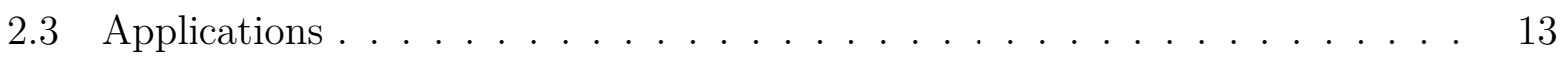

2.3.1 Application 1: ANCOVA Models ............. 13

2.3.2 Application 2: Unreplicated Two-Way Layouts . . . . . . . . . . . . . 16

2.4 Simulation Studies . . . . . . . . . . . . . . . . . . . 18

2.4.1 Simulation Study: ANCOVA Models . . . . . . . . . . . . . . 18

2.4.2 Simulation Study: Unreplicated Two-Way Layouts . . . . . . . . . . 21

2.5 Case Studies . . . . . . . . . . . . . . . . . . . . . 23

2.5.1 Case Study: ANCOVA . . . . . . . . . . . . . . 23

2.5.2 Case Study: Two-Way Unreplicated Layouts . . . . . . . . . . . . . . 23 
2.6 Discussion . . . . . . . . . . . . . . . . . . . . . . . . . . 24

2.7 Supplement . . . . . . . . . . . . . . . . . . . . . . . . 26

2.7.1 Marginal Model Probability Calculations . . . . . . . . . . . . 26

2.7.2 Derivation of Model Probabilities: Noninformative Regression Effect

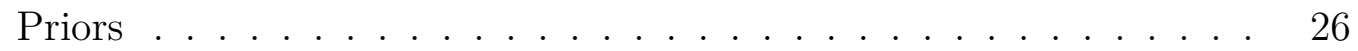

2.7.3 Derivation of Model Probabilities: Mixture $g$ Regression Effect Priors 33

2.7.4 Supplemental ANCOVA Simulation Study . . . . . . . . . . . . 35

2.7.5 Supplemental Two-way Layout Simulation Study . . . . . . . . . . 37

3 Bayesian Model Selection with Group-Based Regression Effects and Heteroscedasticity using the R Package slgf 41

3.1 Introduction . . . . . . . . . . . . . . . . . . . 42

3.1.1 Model Classes and Grouping Schemes . . . . . . . . . . . . . . . . 44

3.1 .2 Classical Approach . . . . . . . . . . . . . . . . . . . . . 46

3.2 Mathematical Details . . . . . . . . . . . . . . . . . . . . . . . . . . . 48

3.2 .1 Model Specification . . . . . . . . . . . . . . . . . . . . . . . . 48

$3.2 .2 \quad$ Parameter Priors . . . . . . . . . . . . . . . . . . . . . . . . . . . . . . . 49

3.2.3 Fractional Bayes Factors and Posterior Model Probabilities . . . . . . 50

$3.2 .4 \quad$ Model Priors . . . . . . . . . . . . . . . . . . . . . . . . 51

3.2 .5 Parameter Estimation _. . . . . . . . . . . . . . . . 51

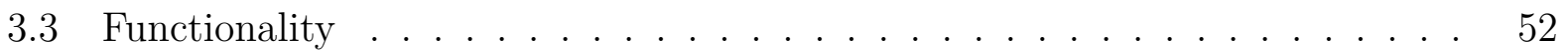

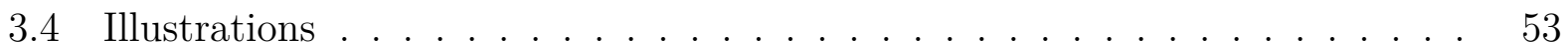




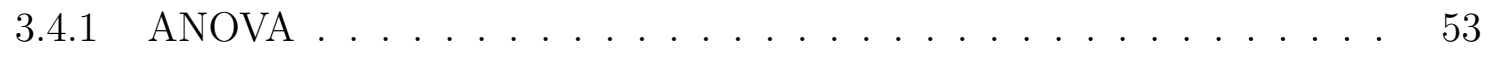

3.4 .2 Analysis of Covariance (ANCOVA) $\ldots \ldots \ldots \ldots$

3.4.3 Two-way Replicated Layouts . . . . . . . . . . . . . . . . 63

3.4.4 Two-way Unreplicated Layouts . . . . . . . . . . . . . . . . . 67

3.4.5 Balanced Incomplete Block Design (BIBD) . . . . . . . . . . 71

3.5 Summary . . . . . . . . . . . . . . . . . . . . 75

4 Conclusions $\quad 76$

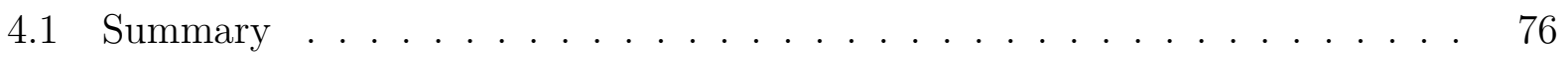

4.2 Future Work . . . . . . . . . . . . . . . . . . 77

$\begin{array}{lr}\text { A Appendix: lm.slgf Code } & 87\end{array}$ 


\section{List of Figures}

2.1 Data from Welch [1951] (left), Flurry [1939] (center), and Franck et al. [2013] (right). After a cursory examination of the data, a researcher might suspect that a latent grouping factor (emphasized by dark and light gray) underlies the levels of the categorical predictor. . . . . . . . . . . . . . . .

2.2 Three possible grouping schemes of the data analyzed by Franck et al. [2013] (as shown in the rightmost panel of Figure 2.1) are shown here. Row membership is used to partition the data into two groups, shaded and unshaded. These grouping schemes are denoted 1,2,3 :4, 5, 6 (left), 1, 2, 4, $6: 3,5$ (center), and $1,4,5: 2,3,6$ (right). . . . . . . . . . .

2.3 Posterior probabilities ( $y$-axis) by class based on 1000 Monte Carlo data sets with $K=4$ levels of the categorical predictor, each with 90 observations, for a total of $N=360$ observations. The true model class is emphasized in bold on the $x$-axis. The dashed line indicates the prior by model class. . . . . .

2.4 Posterior probabilities ( $y$-axis) by class based on 1000 Monte Carlo $10 \times 5$ layouts. The true model class is emphasized in bold on the $x$-axis. The dashed line indicates the prior by model class. . . . . . . . . . . . . . . 
2.5 The most probable models from the Flurry [1939] data set are plotted above: the heteroscedastic group-contracted model (left), with $P\left(m_{1,2: 3}^{\mathrm{VII}} \mid \boldsymbol{Y}\right) \approx 0.51$, and the heteroscedastic group-interaction model (right), with $P\left(m_{1,2: 3}^{\mathrm{VIII}} \mid \boldsymbol{Y}\right) \approx$ 0.42. Overall the grouping scheme where canna and corn are grouped together accounts for about $93.8 \%$ of the posterior model probability, while heteroscedastic models account for about $93.0 \%$ of the posterior model prob-

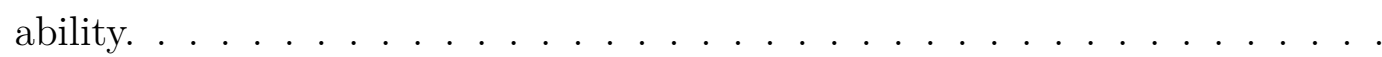

2.6 A two-way unreplicated layout with apparent group-based regression effects [Franck et al., 2013], where $P\left(m_{1,2,5: 3,4,6}^{\mathrm{II}} \mid \boldsymbol{Y}\right) \approx 0.904$ and $P\left(m_{1,2,5: 3,4,6}^{\mathrm{IV}} \mid \boldsymbol{Y}\right) \approx$ 0.064. Overall the grouping scheme 1,2,5:3,4,6 accounts for approximately $96.8 \%$ of the posterior model probability. . . . . . . . . . . . .

2.7 The marginal densities $P\left(\boldsymbol{Y} \mid m_{1: 2,3,4}^{\mathrm{II}}, \lambda_{1}, \lambda_{2}\right)$ and $P^{b}\left(\boldsymbol{Y} \mid m_{1: 2,3,4}^{\mathrm{III}}, \lambda_{1}, \lambda_{2}\right)$ for one particular grouping of an ANOVA layout with $K=4$ levels of $n_{k}=10$ observations each show an overall shape conducive to the Laplace approximation in both the raw and exponentiated likelihood cases. . . . . . . . . . . .

2.8 Posterior probabilities ( $y$-axis) by class based on 1000 Monte Carlo data sets with $K=4$ levels of the categorical predictor, each with 10 observations, for a total of $N=40$ observations. The true model class is emphasized in bold on the $x$-axis. The dashed line indicates the prior by model class. . . . . . .

2.9 Posterior probabilities ( $y$-axis) by class based on 1000 Monte Carlo $10 \times 5$ layouts with smaller effect size. The true model class is emphasized in bold on the $x$-axis. The dashed line indicates the prior by model class. . . . . .

2.10 Posterior probabilities ( $y$-axis) by class based on 1000 Monte Carlo $5 \times 5$ layouts with larger effect size. The true model class is emphasized in bold on the $x$-axis. The dashed line indicates the prior by model class. . . . . . . . . 
2.11 Posterior probabilities ( $y$-axis) by class based on 1000 Monte Carlo $5 \times 5$ layouts with smaller effect size. The true model class is emphasized in bold on the $x$-axis. The dashed line indicates the prior by model class. . . . . . . 40

3.1 Data from O'Brien and Heft [1995] (left), Davies [1954] (center), and Meek and Ozgur [1991] (right). These data sets appear to have a latent grouping structure, color-coded for emphasis. The left panel appears to show two groups with both distinct means and error variances; the center panel shows two groups with distinct regression effects; and the right panel shows groupbased error variances, and group-based interactions may also be present. .

3.2 O'Brien and Heft [1995] studied olfactory function by age ( $y$-axis), where age was divided into five categories ( $x$-axis). The data suggests potential heteroscedasticity, with latent grouping structure $1,2,3: 4,5 \ldots \ldots \ldots$. . .

3.3 The Flurry [1939] data suggests potential heteroscedasticity or a latent group interaction, with scheme potato:corn,canna. . . . . . . . . . . . 59

3.4 An ANCOVA model fit to the Flurry [1939] data leads to a problematic

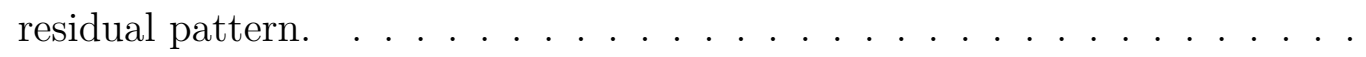

3.5 Residuals on the log-transformed Flurry [1939] data set show an alleviated cone shape, but the heteroscedasticity pattern remains by the levels of starch. 61

3.6 The torque (y-axis) required to tighten a locknut is presented as a function of two predictors. First, the fixture on which the nut was threaded, either a bolt or a conical mandrel, and second, one of three plating methods including cadmium and wax, heat treating, and phosphate and oil, denoted CW, HT, and PO, respectively. Lines connect the means of the plating treatments at each level of fixture. The observations at level bolt appear to have a higher error variance than those of mandrel. 
3.7 The residuals ( $y$-axis) under the full interaction model Torque $\sim$ Fixture+Plating + Fixture*Plating indicate there may be a more subtle heteroscedasticity structure than bolt:mandrel; we color-code for emphasis. We consider Fixture ${ }^{*}$ Plating as the $\operatorname{lgf}$ instead. . . . . . . . . . . . . . . . . 66

3.8 The data analyzed by Franck et al. [2013] measured a continuous gene expression response ( $y$-axis) in six dogs with lymphoma. Tissue samples were taken from tumor and normal tissue in each dog. . . . . . . . . . . . 68

3.9 The data analyzed by Davies [1954] measured the abrasion on four tires (blocks), each manufactured from three of four compounds, in a road wear

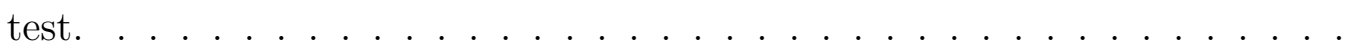

4.1 On closer examination of the Flurry data [Flurry, 1939], it appears that the latent heteroscedasticity by scheme canna,corn:potato can be improved upon if we also model regression effects via the scheme corn:canna,potato. . . . . . 


\section{List of Tables}

2.1 Settings for the eight model classes in the ANCOVA simulation study where $\alpha=0, K=4$, and $N=360 . \ldots \ldots \ldots \ldots$

2.2 Settings for the four model classes in the two-way unreplicated layout simulation study with $10 \times 5$ layouts with larger effect size. . . . . . . . . .

2.3 Settings for the four model classes in the $10 \times 5$ two-way layout simulation study with smaller effect size. . . . . . . . . . . . . . . . . .

2.4 Settings for the four model classes in the two-way layout simulation study with $5 \times 5$ layouts with larger effect size. . . . . . . . . . . . . .

2.5 Settings for the four model classes in the two-way layout simulation study with $5 \times 5$ layouts with smaller effect size. . . . . . . . . . . . 


\section{Chapter 1}

\section{Introduction}

In this work we propose the Suspected Latent Grouping Factor (SLGF) methodology for Bayesian model selection. Our method is applicable to linear models with categorical predictors, an extremely versatile and widely used class of statistical models. We partition the levels of a user-chosen categorical predictor, which we call the SLGF, into two groups. These two groups may elicit a variety of effects on the observed data, including latent groupbased regression effects, group-based interactions, group-based heteroscedasticity, and/or some combination of these structures. Most standard modeling approaches neglect to consider such structures or latent groups, but we demonstrate that they occur quite frequently in common datasets. Our methodology invokes several key components to detect these structures:

1. Combinatoric approach: because a practitioner will often lack prior information on the levels of the categorical predictor that should be grouped together (if they should be grouped together at all), we consider all possible latent grouping structures are considered. See Chapter 2 for further detail.

2. Bayesian model selection: we implement Bayes' Theorem, along with commonly used and effective priors on regression effects and error variances, to quantify the prob- 
ability that specific structures underlie the data in question. This work provides a thorough description of the mathematical and computational approach necessary to compute these model probabilities under our flexible framework, for linear models with a categorical predictor. Thus we can implicitly conduct variable selection as well as select the most probable grouping structures simultaneously. See Chapter 2 for further detail.

3. Fractional Bayes factors; a major advantage of this proposed methodology it that it can compare homoscedastic and heteroscedastic models. An artifact of this feature is that a partial Bayes factor approach must be utilized in order to take full advantage of our objective Bayesian approach. We justify the use of a fractional Bayes factor and demonstrate its utility in our method. See Chapter 2 for further detail.

4. Simulation; we demonstrate the effectiveness of our methodology through a simulation study on several commonly used linear model structures. The study establishes that we can detect a variety of latent grouping structures underlying a dataset. See Chapter 2 for further detail.

5. User-friendly implementation; through the new R package slgf, we thoroughly demonstrate the process a practitioner would invoke to implement our method on a dataset. We demonstrate the functionality of slgf on several linear models that represent a wide range of disciplines, experimental designs, classical analysis methods, and latent grouping structures. This $\mathrm{R}$ package will be publically available on CRAN in the near future. See Chapter 3 for further detail.

It is our hope that this methodology becomes a staple in the analysis of common linear models for researchers in a variety of disciplines. Although that is an ambitious goal, we believe it is realistic for two reasons. First, our method is appropriate in a broad scope of layouts and fields; we illustrate our method's utility in across many datasets representing oneway analysis of variance (ANOVA), replicated and unreplicated two-way layouts, balanced 
incomplete block designs, and analysis of covariance (ANCOVA), in the contexts of biology, agriculture, manufacturing, and engineering. Second, such a method will only be applied by researchers if it is straightforward and accessible to implement for non-statisticians. We believe that we accomplish this with the $\mathrm{R}$ package slgf.

The remainder of this dissertation is organized as follows. Chapter 2 provides a review of previous literature, lays the framework of the statistical model and combinatoric latent grouping approach, summarizes mathematical details on the computation of posterior model probabilities, describes the fractional Bayes factor approach and implementation, analyzes several representative data sets, and gives a simulation study demonstrating the effectiveness of our method. Chapter 3 describes the R package slgf that implements the methodology in practice, by reviewing the approach, providing several examples contrasting a classical approach with our proposed method, and demonstrating the functionality of the package. Chapter 4 summarizes the work and provides several avenues of future research. 


\section{Chapter 2}

\section{Suspected Latent Grouping Factor Methodology}

\subsection{Introduction}

Linear models with categorical predictors are among the most frequently used statistical models, but oversimplification of the variance or regression effect structures can misrepresent key relationships within observed data. Figure 2.1 shows three relevant data sets. First, the left panel shows a simulated one-way ANOVA experiment, based on the statistics reported in Welch [1951]. The horizontal axis represents the levels of a treatment factor, and the vertical axis represents a continuous response. The center panel is an analysis of covariance (ANCOVA) that analyzes the breaking strength of a starch chip [Flurry, 1939]. The horizontal axis represents the chip's thickness in $10^{-4}$ inches, the vertical axis represents the breaking strength in grams, and the point shapes represent the plant from which the starch was derived. Finally, the right panel is an unreplicated two-way layout, measuring the genomic hybridization signal in dogs with lymphoma [Franck et al., 2013]. The horizontal axis represents whether the sample was taken from normal or tumor tissue, the vertical axis represents the intensity of the genomic hybridization signal, and the individual lines represent 
six dogs studied. In each case, the levels of the categorical predictor appear to fall into one of two groups, although the group structure is unknown before data collection. The apparent group structure for the data in Figure 2.1 is represented by dark and light gray. Situations
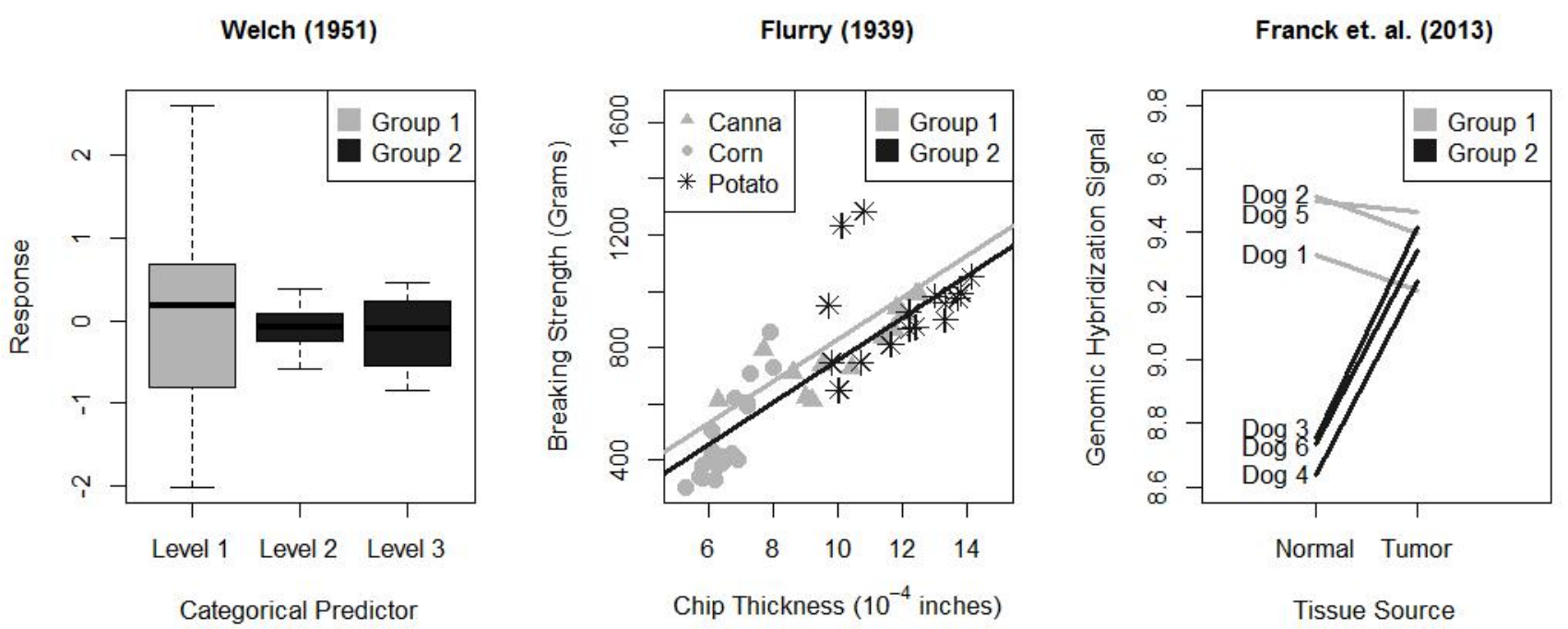

Figure 2.1: Data from Welch [1951] (left), Flurry [1939] (center), and Franck et al. [2013] (right). After a cursory examination of the data, a researcher might suspect that a latent grouping factor (emphasized by dark and light gray) underlies the levels of the categorical predictor.

similar to those illustrated in Figure 2.1 often arise in research. Perhaps after plotting the data or in reviewing previous related work, the researcher begins to suspect that there is a hidden grouping within the levels of the categorical predictor. We call this predictor the suspected latent grouping factor, or SLGF. This work aims to determine whether the latent groupings are plausible and how the group structure affects the response.

We must consider the SLGF with two key ideas in mind: first, that the SLGF might manifest itself through one of several structures in the data, namely, (i) group-specific regression effects, (ii) group-specific variances, and/or (iii) hidden interactions between groups and other model predictors. There are eight possible combinations of structures. Second, the specific assignment of levels to groups is unknown. Both of these aspects of the SLGF must be learned from the data in an unsupervised fashion: specifically, our proposed method 
uses Bayesian model selection to assess the plausibility of the SLGF's impact on the data based on posterior probability. Formal detail can be found in Section 2.

Common linear model assumptions include homoscedasticity and a unique effect of the response at each level of categorical predictors; for a thorough review see Berry [1993]. Violations of these assumptions can have a wide variety of negative consequences on inference; see Rencher and Schaalje [2008], Deschamps [1991] and Scheffé [1959]. Model misspecification can lead to additional problems; see Rencher and Schaalje [2008], Rao [1971], and Deegan $[1976]$.

Regarding the detection of heteroscedasticity, many analyses follow a two-stage approach. These include the methods proposed by Bartlett [1937], Levene [1960], Brown and Forsythe [1974], and Hartley [1950]. When heteroscedasticity is believed to be a function of a continuous predictor, many methods are available, including Breusch and Pagan [1979], Cook and Weisberg [1983], White [1980], Glejser [1969], Park [1966], Box and Hill [1974], Bickel [1978], Jobson and Fuller [1980], and Carroll and Ruppert [1982].

Regarding inference in the presence of heteroscedasticity, methods include those of Box and Cox [1964], Carroll and Ruppert [1988], Perthes [1855], Morrison [1983], Huber [1967], Eicker [1967], Cragg [1983], Hildreth and Houck [1968], Long and Ervin [2000], Polasek et al. [1998], Polasek and Pötzelberger [1994], Cuervo and Achcar [2009], Boscardin and Gelman [1994], MacKinnon and White [1985], Dumitrascu et al. [2015], and White [1980]; for a review see Hayes and Cai [2007] and Cribari-Neto and Zarkos [1999]. Many methods exist to detect heteroscedasticity or conduct inference in its presence. To our knowledge, ours is the first proposal of latent group-based heteroscedasticity alongside possibly unique regression effects and/or hidden interactions.

While an extension to more than two groups is natural, our choice of two groups is still a reasonable approach in many problems; Kharrati-Kopaei and Sadooghi-Alvandi [2007] and Franck [2018] study factor level groupings based on two groups in unreplicated two-way layouts, while Goldfeld and Quandt [1965] model heteroscedasticity as a function of two 
groups, where groups are created by partitioning observations ordinally.

The main contribution of this work is to propose a method of probabilistically detecting the presence of hidden categorical level groupings, and to describe the model specifications that capture the effect of such groupings, through the use of Bayesian model selection. This work generalizes the notion of latent group-based effects from the two-way layout to linear models; see Kharrati-Kopaei and Sadooghi-Alvandi [2007], Franck et al. [2013], Franck and Osborne [2016], and Franck [2018]. There is no unified Bayesian model selection approach to account for these structures in the context of latent groupings of the levels of a categorical predictor in general. Although we illustrate our method in the contexts of the three specific settings shown in Figure 2.1, our proposal is flexible enough to be used in the context of any linear model with a categorical predictor.

The remaining structure of this paper is as follows. Section 2 describes the candidate models in the context of the SLGF, as well as the fractional Bayes factor and Bayesian model selection details. Section 3 describes our proposed Bayesian model specification in contexts of ANCOVA models and unreplicated two-way layouts. Section 4 describes a simulation study to assess the performance of our method. Section 5 applies our proposed method to the empirical data sets of Figure 2.1. Section 6 summarizes the proposed method and provides some additional comments. Additional mathematical details and simulation results for one-way ANOVA data are provided in Section 2.7.

\subsection{Proposed Method}

\subsubsection{Specification of Linear Models with Categorical Predictors}

We begin the development of our approach by elucidating the assignment of the levels of the SLGF into groups. As an illustration, consider the data analyzed by Franck et al. [2013], shown in the right panel of Figure 2.1: an unreplicated two-way layout with 6 rows 
and 2 columns. We choose the dogs as the SLGF. Three examples of possible SLGF level assignments are shown in Figure 2.2: For a SLGF with levels $k=1, \ldots, K+1$, let $\boldsymbol{k}=$

\begin{tabular}{|l|l|l|}
\hline$r_{1}$ & 9.33 & 9.22 \\
\hline$r_{2}$ & 9.51 & 9.39 \\
\hline$r_{3}$ & 8.75 & 9.42 \\
\hline$r_{4}$ & 8.64 & 9.25 \\
\hline$r_{5}$ & 9.50 & 9.46 \\
\hline$r_{6}$ & 8.73 & 9.35 \\
\hline \multicolumn{3}{|l|}{ Example scheme 1} \\
\hline
\end{tabular}

\begin{tabular}{|l|l|l|}
\hline$r_{1}$ & 9.33 & 9.22 \\
\hline$r_{2}$ & 9.51 & 9.40 \\
\hline$r_{3}$ & 8.75 & 9.42 \\
\hline$r_{4}$ & 8.64 & 9.25 \\
\hline$r_{5}$ & 9.50 & 9.46 \\
\hline$r_{6}$ & 8.73 & 9.34 \\
\hline \multicolumn{3}{|c|}{ Example scheme 2} \\
\hline
\end{tabular}

\begin{tabular}{|l|l|l|}
\hline$r_{1}$ & 9.33 & 9.22 \\
\hline$r_{2}$ & 9.51 & 9.39 \\
\hline$r_{3}$ & 8.75 & 9.42 \\
\hline$r_{4}$ & 8.64 & 9.25 \\
\hline$r_{5}$ & 9.50 & 9.46 \\
\hline$r_{6}$ & 8.73 & 9.34 \\
\hline \multicolumn{3}{|c|}{ Example scheme 3 } \\
\hline
\end{tabular}

Figure 2.2: Three possible grouping schemes of the data analyzed by Franck et al. [2013] (as shown in the rightmost panel of Figure 2.1) are shown here. Row membership is used to partition the data into two groups, shaded and unshaded. These grouping schemes are denoted 1,2,3:4,5,6 (left), 1,2, 4,6:3,5 (center), and 1, 4,5:2,3,6 (right).

$(1, \ldots, K+1)^{T}$. We formally define a grouping scheme, denoted $d_{s}(\boldsymbol{k})$, as a partitioning that assigns the data into two groups based on each observation's corresponding level of the SLGF. Let $\mathcal{S}$ be the set of all possible schemes, and $s=1, \ldots, S$ index the possible schemes. We refer to the example scheme 3 , in the rightmost panel of Figure 2.2, to motivate the subsequent notational definitions; this example partitions levels $k=1,4$, and 5 separately from 2, 3, and 6 . For a scheme $d_{s}(\boldsymbol{k})$, a colon separates the levels each group comprises; for example, the scheme of example 3 is denoted as $d_{s}(\boldsymbol{k})=1,4,5: 2,3,6$. The most effective method to partition the levels of the SLGF into groups depends on the size and nature of the study in question. Many problems have $K$ small enough that a combinatoric search over all possible grouping schemes is reasonable; see Franck et al. [2013], Franck and Osborne [2016], Franck [2018], and Kharrati-Kopaei and Sadooghi-Alvandi [2007]. We use the combinatoric search approach exclusively in this study.

Next we formalize the idea of model structures in the context of specific linear models. Recall from Section 2.1 that we must potentially accommodate eight model structures containing a mix of group-based regression effects, group-based variances, and group-based interactions. These structures must be tailored to both the researcher's suspicion and goals, as well as the data layout under consideration. Thus we propose the model class, which is 
the set of models representing a particular structure. While structures reflect the presence or absence of group-based effects, classes prescribe specific corresponding models in the context of the data layout in question.

Now that the grouping schemes and model classes have been enumerated, consider a linear model with $N$ centered observations $\boldsymbol{Y}$ :

$$
\boldsymbol{Y}=X \boldsymbol{\beta}+\varepsilon
$$

with model matrix $X$ parametrized to be full column rank, regression effects $\boldsymbol{\beta}$, and errors $\varepsilon \stackrel{\text { iid }}{\sim} N(\mathbf{0}, \Sigma)$ with covariance matrix $\Sigma$. To account for the eight possible model structures previously described, we will partition $\boldsymbol{Y}, \boldsymbol{\beta}, \boldsymbol{\varepsilon}$, and $\Sigma$. Partition $\boldsymbol{\beta}$ into four components: let $\alpha$ represent an intercept common to all models, let $\boldsymbol{\nu}=\left\{\nu_{k}\right\}_{k=1}^{K}$ represent the SLGF with $K+1$ levels, let $\boldsymbol{\tau}=\left\{\tau_{j}\right\}_{j=1}^{J}$ contain $J$ other regression effects, categorical or continuous, and, let $\boldsymbol{\rho}=\left\{\rho_{\ell}\right\}_{\ell=1}^{L}$ represent $L$ interactions with the SLGF. Then $\boldsymbol{\beta}_{(1+K+J+L) \times 1}=\left(\alpha, \boldsymbol{\nu}_{K \times 1}, \boldsymbol{\tau}_{J \times 1}, \boldsymbol{\rho}_{L \times 1}\right)$.

Similarly, partition the model matrix $X_{N \times(1+K+J+L)}=\left(\mathbf{1}_{N \times 1}^{T}\left|W_{N \times K}\right| V_{N \times J} \mid U_{N \times L}\right)$ into three matrices corresponding to the data related to $\alpha, \boldsymbol{\nu}, \boldsymbol{\tau}$, and $\boldsymbol{\rho}$, respectively. Thus we can express (2.1) equivalently as

$$
\boldsymbol{Y}=\mathbf{1}^{T} \alpha+W \boldsymbol{\nu}+V \boldsymbol{\tau}+U \boldsymbol{\rho}+\boldsymbol{\varepsilon}
$$

In cases where the effect structure for one of the terms in Equation (2.2) depends on a latent grouping scheme, denote that term with a tilde. For example, in structures with group-based interactions, model a group-based interaction $\tilde{\boldsymbol{\rho}}$ instead of usual the interaction $\boldsymbol{\rho}$. Similarly, heteroscedastic structures with group-based variances are modeled with error vector $\tilde{\varepsilon}$ and corresponding covariance matrix $\tilde{\Sigma}$ instead of the homoscedastic counterparts $\varepsilon$ and $\Sigma$.

When a model contains a group structure, we arrange the observations within $\boldsymbol{Y}$ to first contain the $n_{1}$ observations corresponding to $s_{1}$, followed by the $n_{2}$ observations corre- 
sponding to $s_{2}$, denoted $\boldsymbol{Y}=\left(\boldsymbol{Y}_{1}, \boldsymbol{Y}_{2}\right)$. We similarly arrange the rows and columns of $X$ to contain the corresponding observations, which helps concisely express our proposed forms of heteroscedasticity in $\Sigma$. For structures with distinct regression effects by group, arrange $\tilde{\boldsymbol{\nu}}=\left(\tilde{\boldsymbol{\nu}}_{1}, \tilde{\boldsymbol{\nu}}_{2}\right)$, and for classes with distinct variances by group, partition the error vector and covariance matrix such that $\tilde{\varepsilon} \sim N(\mathbf{0}, \tilde{\Sigma})$ where $\tilde{\Sigma}=\left(\begin{array}{c|c}\sigma_{1}^{2} I_{n_{1} \times n_{1}} & 0_{n_{1} \times n_{2}} \\ \hline 0_{n_{2} \times n_{1}} & \sigma_{2}^{2} I_{n_{2} \times n_{2}}\end{array}\right)_{N \times N}$. Notice $N=n_{1}+n_{2}$, for the effects corresponding to groups $s_{1}$ and $s_{2}$, respectively. With multiple schemes, classes, and structures under consideration, we next propose a Bayesian model selection approach to assess whether latent groups exist within the data, and if so, identify the appropriate grouping scheme $d_{s}(\boldsymbol{k}) \in \mathcal{S}$ (if present) and class $c \in \mathcal{C}$.

\subsubsection{Bayesian Model Selection Details}

\section{Model Specification}

Denote the set of all candidate models $\mathcal{M}=\left\{m_{s}^{c}\right\}$, indexed over all possible schemes $s=$ $1, \ldots, S$ and classes $c=1, \ldots, C$, where $|\mathcal{M}|=M$; to ease the notational burden, we have denoted $d_{s}(\boldsymbol{k})$ as $s$ in the subscript of $m$. Although each model matrix $X$ and estimators for $\boldsymbol{\beta}$ and $\Sigma$ depend on the grouping and class under consideration, for notational simplicity we do not index $X, \boldsymbol{\beta}$, or $\Sigma$ by $m, s$, or $c$. Let the vector $\boldsymbol{\varphi}$ contain the single precision $\varphi:=\frac{1}{\sigma^{2}}$ under homoscedastic models, and the corresponding subgroup precisions $\varphi_{1}$ and $\varphi_{2}$ under heteroscedastic models. Denote the precision matrix $\Phi=\Sigma^{-1}$ and $\boldsymbol{\theta}=\{\boldsymbol{\varphi}, \boldsymbol{\beta}\}$, the set of unknown model parameters; then we can express (2.1) conditionally as

$$
\boldsymbol{Y} \mid m_{s}^{c}, \boldsymbol{\theta} \sim N\left(X \boldsymbol{\beta}, \Phi^{-1}\right)
$$

for a given scheme $d_{s}(\boldsymbol{k}) \in \mathcal{S}$ and class $c \in \mathcal{C}$. Thus the likelihood function is given by

$$
P\left(\boldsymbol{Y} \mid \boldsymbol{\theta}, m_{s}^{c}\right)=(2 \pi)^{-\frac{N}{2}}|\Phi|^{\frac{1}{2}} \cdot \exp \left\{-\frac{1}{2}(\boldsymbol{Y}-X \boldsymbol{\beta})^{T} \Phi(\boldsymbol{Y}-X \boldsymbol{\beta})\right\}
$$


We consider two common prior specifications on the regression effects and precision(s). In both cases, we prefer noninformative priors on the precision(s) because prior information on precision is rarely available. First, we consider a noninformative approach, where we have

$$
\begin{gathered}
P\left(\boldsymbol{\beta}, \varphi, \mid m_{s}^{c}\right) \propto \varphi^{-1} \text { (homoscedastic models), or } \\
P\left(\boldsymbol{\beta}, \boldsymbol{\varphi}, \mid m_{s}^{c}\right) \propto \varphi_{1}^{-1} \cdot \varphi_{2}^{-1} \text { (heteroscedastic models) }
\end{gathered}
$$

Next we consider the Zellner-Siow mixture $g$-prior [Zellner and Siow, 1980, Zellner, 1986, Liang et al., 2008], where

$$
\begin{gathered}
P(\alpha, \boldsymbol{\varphi}) \propto \varphi^{-1} \text { (homoscedastic models) } \\
P(\alpha, \boldsymbol{\varphi}) \propto \varphi_{1}^{-1} \cdot \varphi_{2}^{-1} \text { (heteroscedastic models), } \\
\boldsymbol{\beta}_{-\alpha} \mid \boldsymbol{\varphi}, m_{s}^{c} \sim N\left(\mathbf{0}, g\left(X^{T} \Phi^{-1} X\right)^{-1}\right), \text { and } \\
g \sim \operatorname{IG}\left(\frac{1}{2}, \frac{N}{2}\right)
\end{gathered}
$$

where $\boldsymbol{\beta}_{-\alpha}:=\boldsymbol{\beta} \backslash\{\alpha\}$. We use the Zellner-Siow mixture $g$-prior in relatively data-poor situations, such as unreplicated two-way layouts, to reduce the dimensionality of the parameter space with improper priors. This consequently lowers the minimal training sample size, a critical component of the fractional Bayes factor approach described in Section 2.2.2. We use the noninformative flat prior where data are more abundant; see Section 2.3.2 for more detail.

\section{Model Priors for Classes and Schemes}

We impose a uniform model prior by model class: $P\left(m^{c}\right)=\sum_{s=1}^{S} P\left(m_{s}^{c}\right):=\frac{1}{C}$. Depending on the data layout and model structures under consideration, various classes may contain different numbers of models; thus we subsequently divide each class's prior uniformly among 
the models it contains. For example, in an ANOVA layout, one model class might represent the single mean model, where $P\left(m^{c}\right)=\frac{1}{C}$; note we do not index this model by $s$ as there is no grouping structure present in this class. Alternatively, for a model class containing models with distinct regression effects by grouping scheme, the $S$ individual models within the class would be given the prior $P\left(m_{s}^{c}\right)=\frac{1}{S \cdot C}$. By implementing Bayes' Theorem, posterior model probabilities approximated via the fractional Bayes factor (see Section 2.2.2) can then be easily computed for each individual model and class with the marginal probabilities of each model. For a given model $m_{c^{\prime}}^{s^{\prime}}$ we compute:

$$
P\left(m_{s^{\prime}}^{c^{\prime}} \mid \boldsymbol{Y}\right)=\frac{P\left(\boldsymbol{Y} \mid m_{s^{\prime}}^{c^{\prime}}\right) P\left(m_{s^{\prime}}^{c^{\prime}}\right)}{\sum_{c=I s=1}^{C} \sum_{I}^{S} P\left(\boldsymbol{Y} \mid m_{s}^{c}\right) P\left(m_{s}^{c}\right)}
$$

We can then aggregate the overall probability of a given class $c^{\prime}$ as $P\left(m^{c^{\prime}} \mid \boldsymbol{Y}\right)=\sum_{s \in \mathcal{S}} P\left(m_{s}^{c^{\prime}} \mid \boldsymbol{Y}\right)$, or for a given grouping scheme $s^{\prime}$ as $P\left(m_{s^{\prime}} \mid \boldsymbol{Y}\right)=\sum_{c \in \mathcal{C}} P\left(m_{s^{\prime}}^{c} \mid \boldsymbol{Y}\right)$; hence the proposed method allows researchers to draw probabilistic conclusions about both structures and specific grouping schemes.

\section{Fractional Bayes Factor Approach}

We next motivate the need to use a fractional Bayes factor approach. Consider a comparison between arbitrary models $m_{1}$ and $m_{2}$, where $m_{1}$ and $m_{2}$ represent homoscedastic and heteroscedastic models, respectively, via $B^{12}=\frac{P\left(m_{1} \mid \boldsymbol{Y}\right) P\left(m_{2}\right)}{P\left(m_{2} \mid \boldsymbol{Y}\right) P\left(m_{1}\right)}$. For $m_{1}$ we use $P(\boldsymbol{\varphi})=a \cdot \varphi^{-1}$, and for $m_{2}$ we use $P(\boldsymbol{\varphi})=a^{\prime} \cdot \varphi_{1}^{-1} \cdot \varphi_{2}^{-1}$ for arbitrary constants $a \neq a^{\prime}$. So the Bayes factor to compare $m_{1}$ and $m_{2}$ is

$$
B^{12}=\frac{a \cdot \int P\left(\boldsymbol{Y} \mid \boldsymbol{\beta}, \Phi, m_{1}\right) P(\boldsymbol{\beta}) P(\boldsymbol{\varphi}) d \boldsymbol{\beta} d \boldsymbol{\varphi} \cdot P\left(m_{2}\right)}{a^{\prime} \cdot \int P\left(\boldsymbol{Y} \mid \boldsymbol{\beta}, \Phi, m_{2}\right) P(\boldsymbol{\beta}) P(\boldsymbol{\varphi}) d \boldsymbol{\beta} d \boldsymbol{\varphi} \cdot P\left(m_{1}\right)}
$$

which is defined only up to the arbitrary constant $\frac{a}{a^{\prime}}$ and thus inappropriate for use in model comparison. Note this problem arises from the use of noninformative priors on the precisions 
when comparing homoscedastic and heteroscedastic models. Fractional Bayes factors were developed to elicit a cancellation of this constant, rendering a Bayes factor that is well-defined [O’Hagan, 1995].

For a description of fractional Bayes factors (FBFs), see O'Hagan [1995] and ?. We use the FBF approach of O'Hagan rather than the intrinsic Bayes factor of Berger and Pericchi [1996], as the need to choose a training sample would be complicated by the potential scarcity of data induced by some clustering schemes.

To fully quantify the fractional marginal probability of a given model $m_{i}$, we must compute

both $\int P\left(\boldsymbol{Y} \mid \boldsymbol{\theta}_{i}, m_{i}\right) \pi\left(\boldsymbol{\theta}_{i}\right) d \boldsymbol{\theta}_{i}$ and $\int P^{b}\left(\boldsymbol{Y} \mid \boldsymbol{\theta}_{i}, m_{i}\right) \pi\left(\boldsymbol{\theta}_{i}\right) d \boldsymbol{\theta}_{i}$ for some user-chosen fractional exponent b. O'Hagan [1995] provides several recommendations for $b$, including $b:=\frac{m_{0}}{N}$, where $m_{0}$ is the minimal training sample size necessary for $P^{b}\left(\boldsymbol{Y} \mid m_{i}\right)$ to be proper. In this study we choose $b=\frac{m_{0}}{N}$, which elicits consistent model selection [O'Hagan, 1995]. In many cases, tractable expressions exist for both $\int P\left(\boldsymbol{Y} \mid \boldsymbol{\theta}_{i}, m_{i}\right) \pi(\boldsymbol{\theta}) d \boldsymbol{\theta}$ and $\int P^{b}\left(\boldsymbol{Y} \mid \boldsymbol{\theta}_{i}, m_{i}\right) \pi(\boldsymbol{\theta}) d \boldsymbol{\theta}$. When these integrals are intractable, we use the Laplace approximation in the computation of both $\int P\left(\boldsymbol{Y} \mid \boldsymbol{\theta}_{i}, m_{i}\right) \pi(\boldsymbol{\theta}) d \boldsymbol{\theta}$ and $\int P^{b}\left(\boldsymbol{Y} \mid \boldsymbol{\theta}_{i}, m_{i}\right) \pi(\boldsymbol{\theta}) d \boldsymbol{\theta}$, as cubature-based approximation is computationally expensive and our study of the integrand surface indicates a suitable shape. See Section 2.7 for justification and further detail.

\subsection{Applications}

\subsubsection{Application 1: ANCOVA Models}

We first consider an ANCOVA scenario with continuous effect $\boldsymbol{\tau}$ and where the SLGF with $K+1$ levels corresponds to the single categorical predictor effect $\boldsymbol{\nu}$. We let $V_{N \times 1}$ contain the observed continuous covariate and $W_{N \times K}$ be the appropriate categorical effect design matrix. We consider models with and without the interaction effect $\boldsymbol{\rho}$, which governs whether the 
linear trends share a common slope. Thus we begin with the model given by

$$
\boldsymbol{Y}=\mathbf{1}^{T} \alpha+W \boldsymbol{\nu}+V \boldsymbol{\tau}+U \boldsymbol{\rho}+\boldsymbol{\varepsilon}
$$

yielding the likelihood function

$$
P\left(\boldsymbol{Y} \mid m_{s}^{c}, \alpha, \boldsymbol{\nu}, \boldsymbol{\tau}, \boldsymbol{\rho}, \boldsymbol{\varphi}\right)=(2 \pi)^{-\frac{N}{2}}|\Phi|^{\frac{1}{2}} \cdot \exp \left\{-\frac{1}{2}\left(\boldsymbol{Y}-\mathbf{1}^{T} \alpha-W \boldsymbol{\nu}-V \boldsymbol{\tau}-U \boldsymbol{\rho}\right)^{T} \Phi\left(\boldsymbol{Y}-\mathbf{1}^{T} \alpha-W \boldsymbol{\nu}-V \boldsymbol{\tau}-U \boldsymbol{\rho}\right)\right\}
$$

To consider the model without an interaction, let $\boldsymbol{\rho}:=\mathbf{0}$. For models with a group effect, let $\boldsymbol{\nu}:=\tilde{\boldsymbol{\nu}}$; for models with a group-by-continuous predictor interaction, let $\boldsymbol{\rho}:=\tilde{\boldsymbol{\rho}}$; and finally for heteroscedastic models, let $\varepsilon:=\tilde{\varepsilon}$. As an illustration, we consider eight distinct model classes.

1. Class I $\left(m^{\mathrm{I}}\right)$ : the "null" model, with no categorical or continuous covariate effects and homoscedastic error variance, contains 1 model with no grouping schemes; $\boldsymbol{Y}=$ $\mathbf{1}^{T} \alpha+\boldsymbol{\varepsilon}, \boldsymbol{\varepsilon} \sim N\left(\mathbf{0}, \sigma^{2} I\right)$

2. Class II $\left(m^{\mathrm{II}}\right)$ : the "simple linear regression (SLR)" model, with a continuous covariate effect only and homoscedastic error variance, contains 1 model with no grouping schemes; $\boldsymbol{Y}=\mathbf{1}^{T} \alpha+V \boldsymbol{\tau}+\boldsymbol{\varepsilon}, \boldsymbol{\varepsilon} \sim N\left(\mathbf{0}, \sigma^{2} I\right)$

3. Class III $\left(m^{\mathrm{III}}\right)$ : the "ANCOVA" model with categorical and continuous covariate effects and homoscedastic error variance, contains 1 model with no grouping schemes; $\boldsymbol{Y}=\mathbf{1}^{T} \alpha+W \boldsymbol{\nu}+V \boldsymbol{\tau}+\boldsymbol{\varepsilon}, \boldsymbol{\varepsilon} \sim N\left(\mathbf{0}, \sigma^{2} I\right)$

4. Class IV $\left(m_{s}^{\mathrm{IV}}\right)$ : the "group-contracted ANCOVA" model with group and continuous covariate effects and homoscedastic error variance, contains $2^{K-1}-1$ schemes; $\boldsymbol{Y}=$ $\mathbf{1}^{T} \alpha+W \tilde{\boldsymbol{\nu}}+V \boldsymbol{\tau}+\boldsymbol{\varepsilon}, \boldsymbol{\varepsilon} \sim N\left(\mathbf{0}, \sigma^{2} I\right)$

5. Class $\mathrm{V}\left(\mathrm{m}^{\mathrm{V}}\right)$ : the "interaction ANCOVA" model with categorical and continuous covariate effects, level-based interaction, and homoscedastic error variance, contains 1 model with no grouping scheme; $\boldsymbol{Y}=\mathbf{1}^{T} \alpha+W \boldsymbol{\nu}+V \boldsymbol{\tau}+U \boldsymbol{\rho}+\boldsymbol{\varepsilon}, \boldsymbol{\varepsilon} \sim N\left(\mathbf{0}, \sigma^{2} I\right)$ 
6. Class VI $\left(m_{s}^{\mathrm{VI}}\right)$ : the "group-interaction" model, with group and continuous covariate effects, group-based interaction, and homoscedastic error variance, contains $2^{K-1}-1$ schemes; $\boldsymbol{Y}=\mathbf{1}^{T} \alpha+W \tilde{\boldsymbol{\nu}}+V \boldsymbol{\tau}+U \tilde{\boldsymbol{\rho}}+\boldsymbol{\varepsilon}, \boldsymbol{\varepsilon} \sim N\left(\mathbf{0}, \sigma^{2} I\right)$

7. Class VII $\left(m_{s}^{\mathrm{VII}}\right)$ : the "heteroscedastic group-contracted" model (heteroscedastic Class IV), with group and continuous covariate effects and heteroscedastic error variance, contains $2^{K-1}-1$ schemes; $\boldsymbol{Y}=\mathbf{1}^{T} \alpha+W \tilde{\boldsymbol{\nu}}+V \boldsymbol{\tau}+\tilde{\boldsymbol{\varepsilon}}, \tilde{\boldsymbol{\varepsilon}} \sim N\left(\mathbf{0}, \tilde{\Sigma}=\left[\begin{array}{c|c}\sigma_{1}^{2} I_{n_{1} \times n_{1}} & 0_{n_{1} \times n_{2}} \\ \hline 0_{n_{2} \times n_{1}} & \sigma_{2}^{2} I_{n_{2} \times n_{2}}\end{array}\right]_{N \times N}\right)$

8. Class VIII $\left(m_{s}^{\mathrm{VIII}}\right)$ : the "heteroscedastic group-interaction" model (heteroscedastic Class VI), with group and continuous covariate effects, group-based interaction, and heteroscedastic error variance, contains $2^{K-1}-1$ schemes; $\boldsymbol{Y}=\mathbf{1}^{T} \alpha+W \tilde{\boldsymbol{\nu}}+V \boldsymbol{\tau}+$ $U \tilde{\boldsymbol{\rho}}+\tilde{\boldsymbol{\varepsilon}}, \tilde{\boldsymbol{\varepsilon}} \sim N\left(\mathbf{0}, \tilde{\Sigma}=\left[\begin{array}{c|c}\sigma_{1}^{2} I_{n_{1} \times n_{1}} & 0_{n_{1} \times n_{2}} \\ \hline 0_{n_{2} \times n_{1}} & \sigma_{2}^{2} I_{n_{2} \times n_{2}}\end{array}\right]_{N \times N}\right)$

Thus the structure of no group-based effects is represented by Classes I, II, III, and V; group-based regression effects are represented by Classes IV, VI, VII, and VIII; group-based variances are represented by Classes VII and VIII.

We use the FBF approach outlined in Section 2.2.2. Typically, the most complex model considered in an ANCOVA study will have a small minimal training sample size relative to the overall sample size. Thus we choose noninformative priors on the regression effects and precision(s) as described in Equations (2.5) and (2.6), so the marginal density of the data conditional on the model is

$$
P\left(\boldsymbol{Y} \mid m_{s}^{c}\right)=\iint P\left(\boldsymbol{Y} \mid \boldsymbol{\beta}, \boldsymbol{\varphi}, m_{s}^{c}\right) P(\boldsymbol{\beta}) P(\boldsymbol{\varphi}) d \boldsymbol{\beta} d \boldsymbol{\varphi}
$$

This marginal density is analytically integrable over all of the homoscedastic classes defined previously. In heteroscedastic cases (classes VII and VIII), we use a Laplace approximation over the log-variances to approximate $P\left(\boldsymbol{Y} \mid m_{s}^{\mathrm{VII}}\right)$ and $P\left(\boldsymbol{Y} \mid m_{s}^{\mathrm{VIII}}\right)$. We demonstrate the performance of this approach through a simulation study in Section 2.4.1 and through empirical data sets in Section 2.5.1. 


\subsubsection{Application 2: Unreplicated Two-Way Layouts}

Next we consider an unreplicated two-way layout with $R$ rows, $C$ columns, and $N=R \times C$ observations. Because of the unreplicated nature of such a design, the full set of standard interaction effects cannot be incorporated due to insufficient degrees of freedom. Treat the row effects as the SLGF $\boldsymbol{\nu}$, and let $\boldsymbol{\tau}$ contain the column effects; note by transposing the data table we could treat the column effects as the SLGF as well.

Our choice of model classes is based on the idea of hidden additivity, where interactions are treated as a group-by-column effect [Franck et al., 2013]. The usual "additive" model $\boldsymbol{Y}=\mathbf{1}^{T} \alpha+W \boldsymbol{\nu}+V \boldsymbol{\tau}+\boldsymbol{\varepsilon}$ accounts for only row and column main effects. The groupbased model, partitioned by levels of the row effect, does not include column effects but does consider group-by-column interactions, denoted by $\tilde{\boldsymbol{\rho}}$. We require at least 2 levels of $k$ (rows) in both groups to ensure there are enough degrees of freedom to estimate this group-by-column interaction. We thus begin with the model

$$
\boldsymbol{Y}=\mathbf{1}^{T} \alpha+W \boldsymbol{\nu}+V \boldsymbol{\tau}+U \tilde{\boldsymbol{\rho}}+\boldsymbol{\varepsilon}
$$

where we let $\tilde{\boldsymbol{\rho}}:=\mathbf{0}$ in the additive model, $\boldsymbol{\tau}:=\mathbf{0}$ in cases with scheme-based regression effects, and $\varepsilon:=\tilde{\varepsilon}$ in cases with group-based heteroscedasticity. So the full likelihood function is given by

$P\left(\boldsymbol{Y} \mid m_{s}^{c}, \alpha, \boldsymbol{\nu}, \boldsymbol{\tau}, \tilde{\boldsymbol{\rho}}, \boldsymbol{\varphi}\right)=(2 \pi)^{-\frac{N}{2}}|\Phi|^{\frac{1}{2}} \exp \left\{-\frac{1}{2}(Y-\mathbf{1} \alpha-W \boldsymbol{\nu}-V \boldsymbol{\tau}-U \tilde{\boldsymbol{\rho}})^{T} \Phi(Y-\mathbf{1} \alpha-W \boldsymbol{\nu}-V \boldsymbol{\tau}-U \tilde{\boldsymbol{\rho}})\right\}$

In this layout we consider four model classes:

1. Class I $\left(m^{\mathrm{I}}\right)$ : the "additive" model, where column effects are equivalent across rows and error variance is constant across all observations, contains 1 model with no grouping schemes; $\boldsymbol{Y}=\mathbf{1}^{T} \alpha+W \boldsymbol{\nu}+V \boldsymbol{\tau}+\boldsymbol{\varepsilon}, \boldsymbol{\varepsilon} \sim N\left(\mathbf{0}, \sigma^{2} I\right)$

2. Class II $\left(m_{s}^{\mathrm{II}}\right)$ : the "group-by-column interaction" model, where levels are divided into 
two latent groups based on grouping scheme $d_{s}(\boldsymbol{k})$ with distinct means and equivalent variance, contains $2^{K-1}-K-1$ grouping schemes [Franck et al., 2013]; $\boldsymbol{Y}=\mathbf{1}^{T} \alpha+$ $W \boldsymbol{\nu}+U \tilde{\boldsymbol{\rho}}+\boldsymbol{\varepsilon}, \boldsymbol{\varepsilon} \sim N\left(\mathbf{0}, \sigma^{2} I\right)$

3. Class III $\left(m_{s}^{\mathrm{III}}\right)$ : the "heteroscedastic additive" model, where levels are divided into two latent groups based on grouping scheme $d_{s}(\boldsymbol{k})$ with equivalent means and group-based variances, contains $2^{K-1}-K-1$ grouping schemes; $\boldsymbol{Y}=\mathbf{1}^{T} \alpha+W \boldsymbol{\nu}+V \boldsymbol{\tau}+\tilde{\boldsymbol{\varepsilon}}, \tilde{\boldsymbol{\varepsilon}} \sim$ $N\left(\mathbf{0}, \tilde{\Sigma}=\left[\begin{array}{c|c}\sigma_{1}^{2} I_{n_{1} \times n_{1}} & 0_{n_{1} \times n_{2}} \\ \hline 0_{n_{2} \times n_{1}} & \sigma_{2}^{2} I_{n_{2} \times n_{2}}\end{array}\right]_{N \times N}\right)$

4. Class IV $\left(m_{s}^{\mathrm{IV}}\right)$ : the "heteroscedastic group-by-column interaction" model where levels are divided into two latent groups based on grouping scheme $d_{s}(\boldsymbol{k})$ with distinct means and group-based variances, contains $2^{K-1}-K-1$ grouping schemes; $\boldsymbol{Y}=\mathbf{1}^{T} \alpha+W \boldsymbol{\nu}+$ $U \tilde{\boldsymbol{\rho}}+\tilde{\boldsymbol{\varepsilon}}, \tilde{\boldsymbol{\varepsilon}} \sim N\left(\mathbf{0}, \tilde{\Sigma}=\left[\begin{array}{c|c}\sigma_{1}^{2} I_{n_{1} \times n_{1}} & 0_{n_{1} \times n_{2}} \\ \hline 0_{n_{2} \times n_{1}} & \sigma_{2}^{2} I_{n_{2} \times n_{2}}\end{array}\right]_{N \times N}\right)$

Thus there are $M=\left(3 \times 2^{K-1}\right)-3 K-2$ models considered.

\section{Bayesian Model Specification: Unreplicated Two-Way Layouts}

The two-way unreplicated layout is typically modeled with a high ratio of parameters to data points. Thus we must take care in choosing priors that allow us to successfully incorporate the $\mathrm{FBF}$ approach. With noninformative priors on the regression effects, the minimal training sample size needed to estimate $R-1$ regression effects, $2 \cdot(C-1)$ group-by-column interactions, and 2 error variances would be prohibitively large in relation to the sample size $N$; indeed, $b=\frac{m_{0}}{N}<\frac{1}{2}$ only when $R \geq 7$. In this work, we propose using the Zellner-Siow mixture $g$-prior on regression coefficients to reduce the dimensionality of the improper prior. Our use of this automatic prior on the regression effects lowers the minimal training sample size to $m_{0}=3$, and thus the fractional exponent $b=\frac{m_{0}}{N}$, to a value that allows us to 
successfully implement the FBF. Thus we let

$$
\begin{gathered}
P(\alpha) \propto 1, \\
P(\boldsymbol{\beta} \mid \boldsymbol{\varphi}, g)=N\left(\mathbf{0}, g\left(X^{T} \Phi X\right)^{-1}\right), \\
P(\boldsymbol{\varphi}) \propto \varphi^{-1} \text { (for models with homoscedasticity), } \\
P(\boldsymbol{\varphi}) \propto \varphi_{1}^{-1} \varphi_{2}^{-1} \text { (for models with heteroscedasticity), and } \\
P(g)=\mathrm{IG}\left(\frac{1}{2}, \frac{N}{2}\right)
\end{gathered}
$$

The marginal density of the data conditional on the model is

$$
P\left(\boldsymbol{Y} \mid m_{s}^{c}\right)=\iiint \int P\left(\boldsymbol{Y} \mid \alpha, \boldsymbol{\beta}, \boldsymbol{\varphi}, m_{s}^{c}\right) P(\alpha) P(\boldsymbol{\beta} \mid \boldsymbol{\varphi}, g) P(\boldsymbol{\varphi}) P(g) d \alpha d \boldsymbol{\beta} d \boldsymbol{\varphi} d g
$$

It is well-known in the homoscedastic case that the Zellner-Siow mixture $g$-prior advantageously elicits a Cauchy distribution on the regression effects [Liang et al., 2008]. We show the Cauchy result also holds in the heteroscedastic case; that is:

$$
\boldsymbol{\beta} \sim \operatorname{MVCauchy}_{p}\left(\text { location }=0 \text {, scale }=\left(\frac{X^{T} \Phi X}{n}\right)^{-1}\right) ; \text { see Section } 2.7 \text { for proof. }
$$

In classes with homoscedasticity, this intergral is intractable over $g$ and thus a Laplace approximation is conducted over a single dimension; in heteroscedastic cases, a three-dimensional Laplace approximation is used to integrate $g, \lambda_{1}=\log \left(\varphi_{1}\right)$, and $\lambda_{2}=\log \left(\varphi_{2}\right)$.

\subsection{Simulation Studies}

\subsubsection{Simulation Study: ANCOVA Models}

In order to simulate ANCOVA data, we generated independent draws $x$ uniformly over the interval $(0,10)$. Outcomes $\boldsymbol{Y}$ were then simulated according to each of the eight classes 


\begin{tabular}{|c|c|}
\hline Class & Parameters \\
\hline I (Null) & $\sigma^{2}=1$ \\
\hline II (SLR) & $\tau=0.5, \sigma^{2}=1$ \\
\hline III (ANCOVA) & $\alpha=2.0, \nu=(4.0,6.0,8.0), \tau=0.5, \sigma^{2}=1$ \\
\hline IV (Group-Contracted ANCOVA) & $\alpha=0.0, \tilde{\nu}=(3.0), \tau=0.5, \sigma^{2}=1$ \\
\hline V (Interaction ANCOVA) & $\alpha=0.5, \nu=(1.0,1.5,2.0), \rho=(0.25,0.5,0.75,1.0), \tau=0.5, \sigma^{2}=1$ \\
\hline VI (Group-Interaction ANCOVA) & $\alpha=0.0, \tilde{\nu}=(0.8), \tilde{\rho}=(0,1), \tau=1, \sigma^{2}=1$ \\
\hline VII (Heteroscedastic & $\alpha=0.0, \tilde{\nu}=(3.0), \tau=0.5$, \\
Group-Contracted ANCOVA) & $\sigma_{1}^{2}=1, \sigma_{2}^{2}=5$ \\
\hline VIII (Heteroscedastic & $\alpha=0.0, \tilde{\nu}=(3.0), \tau=0.5, \tilde{\rho}=(0,1)$, \\
Group-Interaction ANCOVA) & $\sigma_{1}^{2}=1, \sigma_{2}^{2}=5$ \\
\hline
\end{tabular}

Table 2.1: Settings for the eight model classes in the ANCOVA simulation study where $\alpha=0, K=4$, and $N=360$.

described in Section 2.3.1 with continuous, categorical, interaction, and/or group-based effects, as well as errors with group-based heteroscedasticity, as appropriate. In this study, we simulated $n_{k}=90$ observations at each level of the SLGF; another simulation study with $n_{k}=10$ is provided in Section 2.7. We note that the settings in Table 2.1 are not calibrated to be equivalent in the total effect between classes, where such a calibration would be nontrivial. For example, the Class $\mathrm{V}$ model contains eight non-null parameters compared to the Class I model's single parameter; thus we avoid comparing overall performance between classes in this study.

Figure 2.8 shows that overall our method accurately attributes posterior model probability to the correct model class for the eight classes described in Section 2.3.1. For Class I (null model) data, the posterior probability of the true class is high relative to the other classes, indicating that our method neither misattributes noise to a group-based structure, nor appears to systematically favor one of the other model classes when it does select the wrong model. Class II (SLR model) data performs similarly, but Classes IV (group-contracted ANCOVA) and VII (group-interaction) capture some posterior probability as well. The Class III (ANCOVA) data setting is favored by the correct class, with Class V (interaction ANCOVA) being the second choice. Class IV (group-contracted ANCOVA) data appears to be the most difficult to detect based on the parameters chosen for this study; although its 

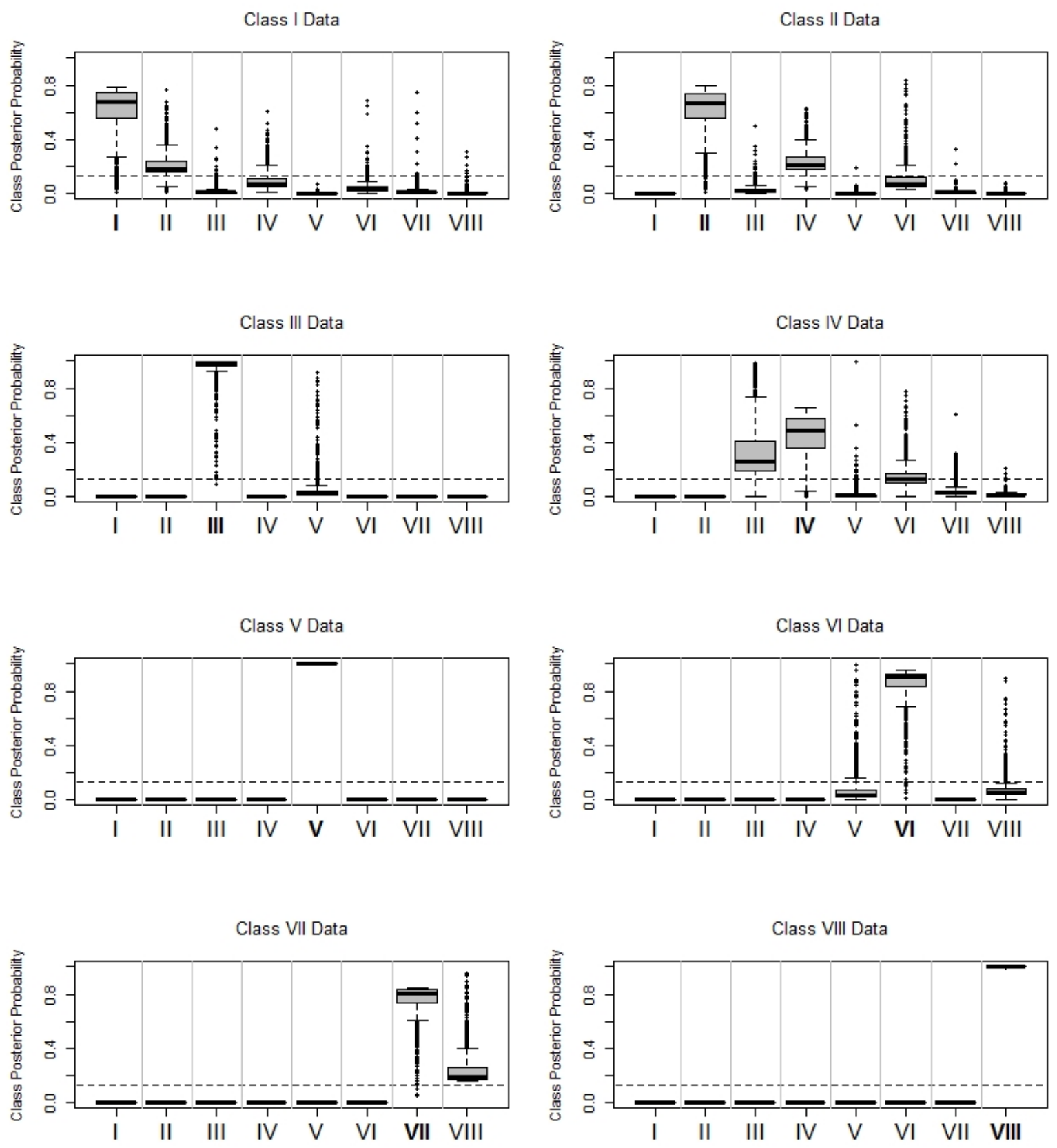

Figure 2.3: Posterior probabilities ( $y$-axis) by class based on 1000 Monte Carlo data sets with $K=4$ levels of the categorical predictor, each with 90 observations, for a total of $N=360$ observations. The true model class is emphasized in bold on the $x$-axis. The dashed line indicates the prior by model class. 
posterior probabilities are generally higher than the other classes', it is often mistaken for Class III data, meaning in this case a group-based categorical effect is often mistaken for the full categorical effect. Note that these misattributed classes differ by only two parameters in the regression structure. For Class V (interaction ANCOVA) data, the correct model class is favored. Class VI (group-interaction) data is also correctly favored the majority of the time; when misclassified, it is usually chosen as Class V (interaction ANCOVA) or VIII (heteroscedastic ANCOVA). Classes VII (heteroscedastic group-contracted) and VIII (heteroscedastic group-interaction) perform well; when wrong, Class VII tends to favor a spurious interaction effect.

These results indicate that, in general, our method tends to identify the correct class. When no group-based structure is present, our method tends to not erroneously fit spurious effects or variances. Thus group-based regression effects, interactions, and/or variances are detected with high accuracy when present. We see similar performance with the additional study provided in Section 2.7 where $N=40$.

\subsubsection{Simulation Study: Unreplicated Two-Way Layouts}

Row and column effects along with error variance(s) (provided in Table 2.2) were simulated to generate unreplicated two-way layouts. We consider layouts of size $10 \times 5$ where $N=50$. Another setting with a smaller effect size, and a study on layouts of size $5 \times 5$, are given in Section 2.7.

Figure 2.4 shows that our method also tends to favor the true class in the two-way unreplicated layout, with high parameter to data ratio and the mixture $g$-prior. For additive data from Class I, the true class is generally favored; the most probable alternative is typically the heteroscedastic additive Class III. Under large effect size, Class II (group-by-column interaction) data is correctly identified. Class III (heteroscedastic additive) and Class IV 


\begin{tabular}{|c|c|}
\hline Class & Parameters \\
\hline I (Additive Model) & $\alpha=1, \boldsymbol{\nu} \in\{2,3,4,5,6,7,8,9,10\}, \boldsymbol{\tau} \in\{1,2,3,4,5\}, \sigma^{2}=1$ \\
\hline $\begin{array}{l}\text { II (Group-by-Column } \\
\text { Interaction) }\end{array}$ & $\begin{array}{c}\alpha=1, \boldsymbol{\nu} \in\{2,3,4,5,6,7,8,9,10\}, \boldsymbol{\tau}_{1} \in\{1.0,1.8,2.6,3.4,4.2\} \\
\boldsymbol{\tau}_{2} \in\{4.2,3.4,2.6,1.8,1.0\}, \sigma^{2}=1\end{array}$ \\
\hline $\begin{array}{l}\text { III (Heteroscedastic } \\
\text { Additive) }\end{array}$ & $\begin{array}{c}\alpha=1, \boldsymbol{\nu} \in\{2,3,4,5,6,7,8,9,10\}, \boldsymbol{\tau} \in\{1,2,3,4,5\} \\
\sigma_{1}^{2}=1.0, \sigma_{2}^{2}=0.10\end{array}$ \\
\hline $\begin{array}{l}\text { IV (Heteroscedastic Group- } \\
\text { by-Column Interaction) }\end{array}$ & $\begin{array}{c}\alpha=1, \boldsymbol{\nu} \in\{2,3,4,5,6,7,8,9,10\}, \boldsymbol{\tau}_{1} \in\{1.0,1.8,2.6,3.4,4.2\} \\
\boldsymbol{\tau}_{2} \in\{4.2,3.4,2.6,1.8,1.0\}, \sigma_{1}^{2}=1.0, \sigma_{2}^{2}=0.10\end{array}$ \\
\hline
\end{tabular}

Table 2.2: Settings for the four model classes in the two-way unreplicated layout simulation study with $10 \times 5$ layouts with larger effect size.
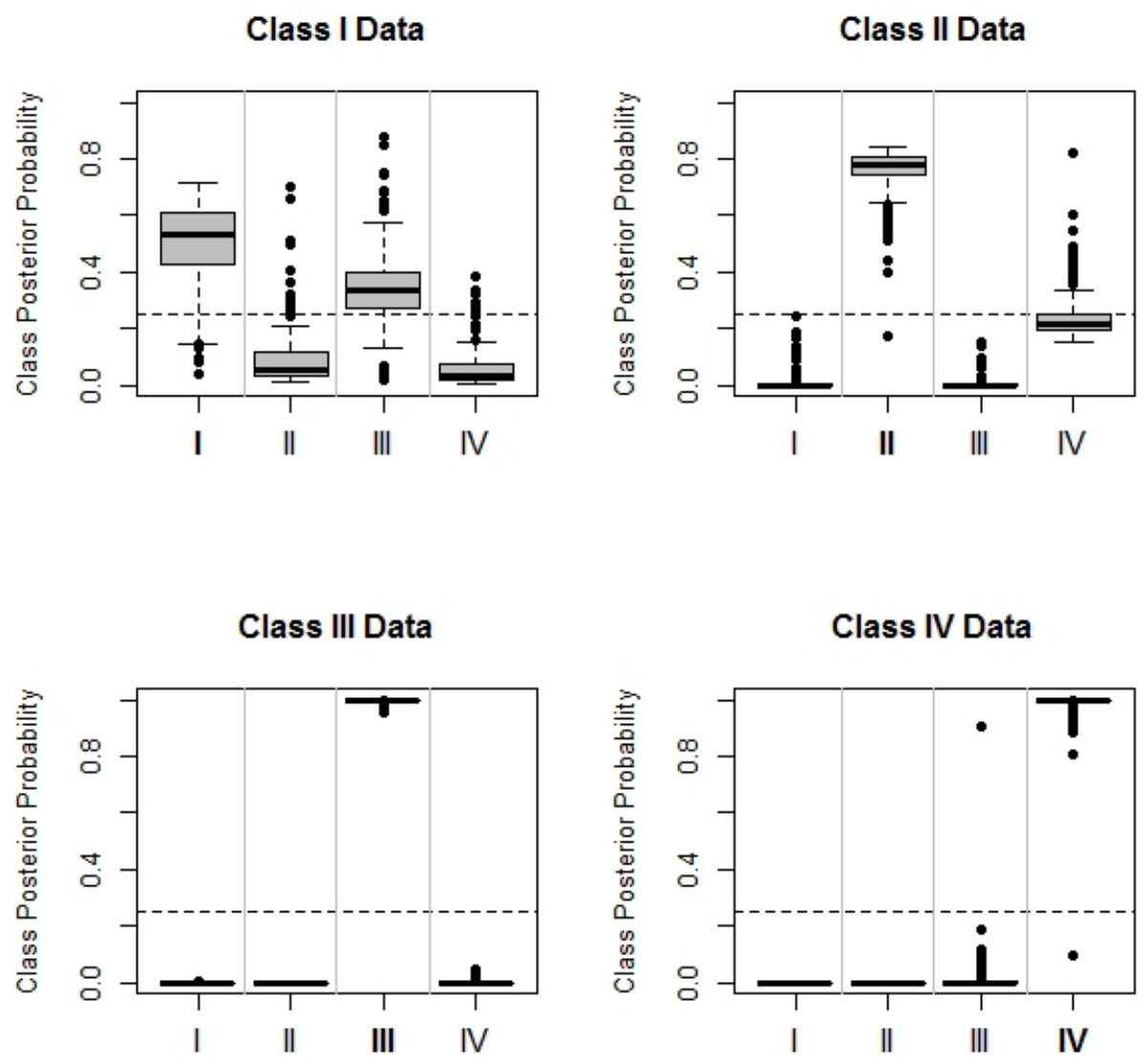

Figure 2.4: Posterior probabilities ( $y$-axis) by class based on 1000 Monte Carlo $10 \times 5$ layouts. The true model class is emphasized in bold on the $x$-axis. The dashed line indicates the prior by model class. 
(heteroscedastic group-by-column interaction) data are both favored correctly as well regarding both effect sizes.

\subsection{Case Studies}

\subsubsection{Case Study: ANCOVA}

We revisit the Flurry [1939] data, shown in the center panel of Figure 2.1 and in Figure 2.5. The breaking strength of a chip coated with a film derived from one of three plant materials is studied as a function of a continuous predictor (the thickness of the film) and a categorical predictor (the plant type from which the film was developed). The plot seems to indicate some degree of heteroscedasticity between canna and corn, versus potato; results are shown in Figure 2.5. Two models receive $93 \%$ of the posterior probability: the heteroscedastic group-contracted model, with $P\left(m_{1,2: 3}^{\mathrm{VII}} \mid \boldsymbol{Y}\right) \approx 0.51$, and the heteroscedastic group-interaction model, with $P\left(m_{1,2: 3}^{\mathrm{VIII}} \mid \boldsymbol{Y}\right) \approx 0.42$.

\subsubsection{Case Study: Two-Way Unreplicated Layouts}

Franck et al. [2013] and Franck and Osborne [2016] examine a two-way unreplicated layout describing the copy number variation for genes in six dogs with lymphoma. Samples were taken from healthy and diseased tissue within each dog. It is clear in the plot that dogs behave differently by group: dogs 1,2 , and 5 appear to behave distinctly from dogs 3,4 , and 6. With six subjects by row, there are $1+3 \cdot\left(2^{6-1}-6-1\right)=76$ candidate models, including the null model and three classes each containing 25 models. Thus we show only the six most probable models, which account for $98.1 \%$ of the posterior probability; these results are shown in Figure 2.6. We conclude with high probability that subjects 1, 2, and 5 behave distinctly from 3, 4, and 6 ; this scheme over all classes has probability $P\left(m_{1,2,5: 3,4,6} \mid \boldsymbol{Y}\right) \approx .9680$. More specifically, we also conclude homoscedastic behavior along with this grouping scheme, the 
Class VII 1,2:3 (Heteroscedastic Group-Contracted)

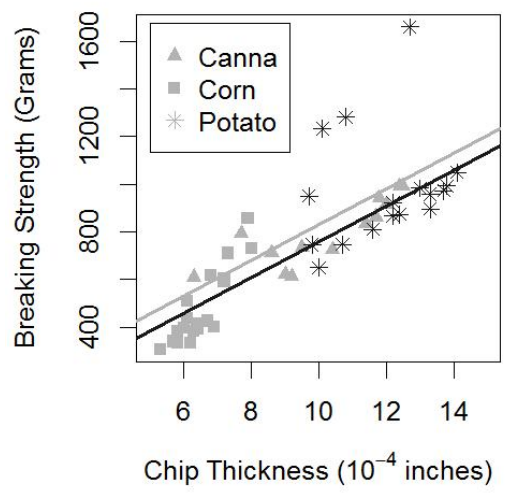

Class VIII 1,2:3 (Heteroscedastic Group-Interaction)

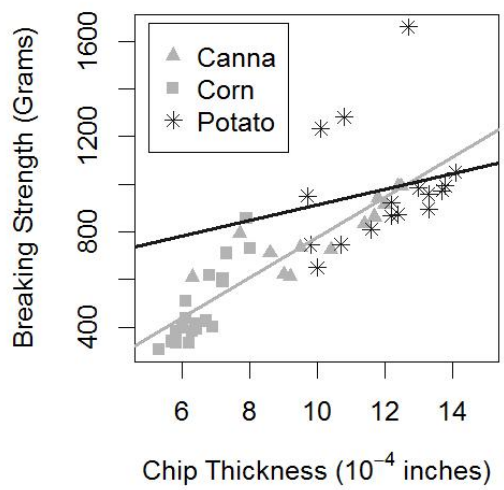

Figure 2.5: The most probable models from the Flurry [1939] data set are plotted above: the heteroscedastic group-contracted model (left), with $P\left(m_{1,2: 3}^{\mathrm{VII}} \mid \boldsymbol{Y}\right) \approx 0.51$, and the heteroscedastic group-interaction model (right), with $P\left(m_{1,2: 3}^{\mathrm{VII}} \mid \boldsymbol{Y}\right) \approx 0.42$. Overall the grouping scheme where canna and corn are grouped together accounts for about $93.8 \%$ of the posterior model probability, while heteroscedastic models account for about $93.0 \%$ of the posterior model probability.

group-by-column interaction model, with $P\left(m_{1,2,5: 3,4,6} \mid \boldsymbol{Y}\right) \approx .9040$.

\subsection{Discussion}

Our proposed method is a flexible and intuitive approach to accommodate linear models with latent group-based effects underlying the data. This method generalizes the homoscedastic, two-way unreplicated layout work of Franck [2018] to a heteroscedastic approach to any linear model with a categorical predictor. By partitioning the data according to the levels of a categorical predictor, we can detect latent structures within the data that might influence the regression effects, error variance, or interaction effects. Often, these group-based structures have straightforward and intuitive interpretations in the context of the data, making the approach particularly useful to domain experts. The use of fractional Bayes factors allows us to compare homoscedastic and heteroscedastic models with minimal prior influence. Regarding priors on the regression effects, we explored the performance of noninformative and 

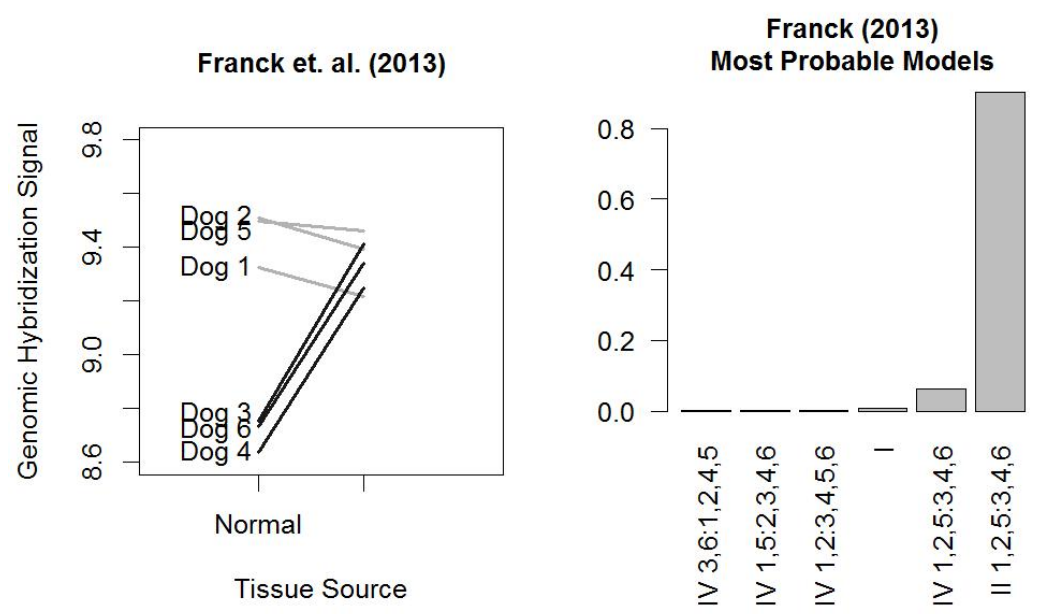

Figure 2.6: A two-way unreplicated layout with apparent group-based regression effects [Franck et al., 2013], where $P\left(m_{1,2,5: 3,4,6}^{\mathrm{II}} \mid \boldsymbol{Y}\right) \approx 0.904$ and $P\left(m_{1,2,5: 3,4,6}^{\mathrm{IV}} \mid \boldsymbol{Y}\right) \approx 0.064$. Overall the grouping scheme 1,2,5:3,4,6 accounts for approximately $96.8 \%$ of the posterior model probability.

mixture $g$-priors in the contexts of ANCOVA and two-way unreplicated layouts, but many other choices on priors and data layouts can be considered. We considered cases in which the number of levels of the SLGF $K$ was relatively small, so that a combinatoric search over all possible grouping schemes was computationally feasible. In cases where $K$ is large, a Markov-chain model composition (MC3) could be used to search the model space.

In some cases, our method leads to a contraction of the number of effects modeled. For instance, in the ANCOVA examples illustrated in Section 2.3.1, estimating a group effect $\tilde{\boldsymbol{\nu}}$ as opposed to a categorical effect $\boldsymbol{\nu}$ will reduce the degrees of freedom used to estimate the effect from $K$ to 2 . Alternatively, in the two-way unreplicated layout examples, modeling a group-by-column interaction rather than column effects will expand the degrees of freedom used from $C-1$ to $2 \cdot(C-1)$.

While we have illustrated our method using two latent groups, an extension to three or more groups is straightforward conceptually but increases the complexity of the models, the number of model classes, and the number of models to consider. Fortunately, the two-group assumption has been shown to lead to useful inferences in several previous works, including 
Kharrati-Kopaei and Sadooghi-Alvandi [2007], Franck et al. [2013], Franck and Osborne [2016], and Franck [2018].

\subsection{Supplement}

\subsubsection{Marginal Model Probability Calculations}

To prevent numeric underflow we consider model probabilites on the logarithmic scale in all cases. Consider the set of all log-marginal probabilities $\mathcal{L}=\left\{\log P\left(Y \mid m_{s}^{c}\right)\right\}$. Let $\ell^{\star}=\max \mathcal{L}$ and $m^{\star}=\arg \max \mathcal{L}$. Transform $\mathcal{L}^{\star}=\mathcal{L}-\ell^{\star}$ to obtain the set $\exp \left[\mathcal{L}^{\star}\right]=\left\{\exp \left[\log P\left(Y \mid m_{s}^{c}\right)-\right.\right.$ $\left.\left.\log P\left(Y \mid m^{\star}\right)\right]\right\}=\left\{\frac{P\left(Y \mid m_{s}^{c}\right)}{P\left(Y \mid m^{\star}\right)}\right\}=\left\{B^{m_{s}^{c}, m^{\star}}\right\}$, representing Bayes factors for each model relative to the most probable model. Note the untransformed model probabilities are given by $P=\left\{\frac{P\left(Y \mid m_{s}^{c}\right) P\left(m_{s}^{c}\right)}{\sum P\left(Y \mid m_{s}^{c}\right) P\left(m_{s}^{c}\right)}\right\}=\left\{\frac{P\left(Y \mid m_{s}^{c}\right) P\left(m_{s}^{c}\right) / P\left(Y \mid m^{\star}\right)}{\sum P\left(Y \mid m_{s}^{c}\right) P\left(m_{s}^{c}\right) / P\left(Y \mid m^{\star}\right)}\right\}=\left\{\frac{B^{m_{s}^{c}, m^{\star}} P\left(m_{s}^{c}\right)}{\sum B^{m_{s}^{c}, m^{\star}} P\left(m_{s}^{c}\right)}\right\}$; thus we can easily use these transformed log-marginal probabilities to obtain posterior model probabilities. Our Laplace approximations generally provided values comparable to quadrature and cubaturebased approximations at less computational expense.

\subsubsection{Derivation of Model Probabilities: Noninformative Regres- sion Effect Priors}

Let $H_{X}:=X\left(X^{T} X\right)^{-1} X^{T}$. 
Homoscedastic Error Variance, No Group-Based Regression Effects

$$
\begin{aligned}
& P(\boldsymbol{Y} \mid m)=\int_{\Theta}\left[(2 \pi)^{-\frac{N}{2}}|\Sigma|^{-\frac{1}{2}} \cdot \exp \left\{-\frac{1}{2}(\boldsymbol{Y}-X \boldsymbol{\beta})^{T} \Sigma^{-1}(\boldsymbol{Y}-X \boldsymbol{\beta})\right\}\right]^{b} P(\boldsymbol{\theta}) d \boldsymbol{\theta} \\
& =\int_{0}^{\infty} \int_{-\infty}^{\infty}(2 \pi)^{-\frac{N b}{2}}|\Sigma|^{-\frac{b}{2}} \exp \left\{-b \cdot \frac{1}{2}\left[\boldsymbol{Y}^{T} \Sigma^{-1} \boldsymbol{Y}-2 \boldsymbol{\beta}^{T} X^{T} \Sigma^{-1} \boldsymbol{Y}+\boldsymbol{\beta}^{T} X^{T} \Sigma^{-1} X \boldsymbol{\beta}\right]\right\} \Sigma^{-1} d \boldsymbol{\beta} d \gamma \\
& =\int_{0}^{\infty} \int_{-\infty}^{\infty}(2 \pi)^{-\frac{N b}{2}} \gamma^{\frac{N b}{2}-1} \exp \left\{-b \cdot \frac{1}{2}\left[\boldsymbol{Y}^{T} \Sigma^{-1} \boldsymbol{Y}-2 \boldsymbol{\beta}^{T} X^{T} \Sigma^{-1} \boldsymbol{Y}+\boldsymbol{\beta}^{T} X^{T} \Sigma^{-1} X \boldsymbol{\beta}\right]\right\} d \boldsymbol{\beta} d \gamma \\
& =\int_{0}^{\infty} \int_{-\infty}^{\infty}(2 \pi)^{-\frac{N b}{2}} \gamma^{\frac{N b}{2}-1} \exp \left\{-b \cdot \frac{\gamma}{2}\left[\boldsymbol{\beta}^{T} X^{T} X \boldsymbol{\beta}-2 \boldsymbol{\beta}^{T} X^{T} \boldsymbol{Y}+\boldsymbol{Y}^{T} H \boldsymbol{Y}\right]\right\} \times \\
& \exp \left\{-b \cdot \frac{\gamma}{2}\left[\boldsymbol{Y}^{T} \boldsymbol{Y}-\boldsymbol{Y}^{T} H \boldsymbol{Y}\right]\right\} d \boldsymbol{\beta} d \gamma \\
& =\int_{0}^{\infty}(2 \pi)^{-\frac{N b}{2}} \gamma^{\frac{N b}{2}-1}(2 \pi)^{+\frac{p}{2}}\left|b^{-1} \gamma^{-1}\left(X^{T} X\right)^{-1}\right|^{+\frac{1}{2}} \exp \left\{-b \cdot \frac{\gamma}{2}\left[\boldsymbol{Y}^{T}(I-H) \boldsymbol{Y}\right]\right\} d \gamma \\
& =(2 \pi)^{-\frac{N b-P}{2}} b^{-\frac{P}{2}}\left|X^{T} X\right|^{-\frac{1}{2}} \Gamma\left(\frac{N b-P}{2}\right)\left(\frac{b \cdot \operatorname{SSResid}^{\mathrm{I}}}{2}\right)^{-\frac{N b-P}{2}} \\
& =\pi^{-\frac{N b-P}{2}} b^{-\frac{N b}{2}}\left|X^{T} X\right|^{-\frac{1}{2}} \Gamma\left(\frac{N b-P}{2}\right)\left(\text { SSResid }^{\mathrm{I}}\right)^{-\frac{N b-P}{2}} \text {. } \\
& q^{b}(\boldsymbol{Y} \mid m)=\frac{\int_{\Theta} P\left(Y \mid m^{\mathrm{I}}, \boldsymbol{\theta}\right) P(\boldsymbol{\theta}) d \boldsymbol{\theta}}{\int_{\Theta} P^{b}\left(Y \mid m^{\mathrm{I}}, \boldsymbol{\theta}\right) P(\boldsymbol{\theta}) d \boldsymbol{\theta}} \\
& =\frac{\pi^{-\frac{N-P}{2}}\left|X^{T} X\right|^{-\frac{1}{2}} \Gamma\left(\frac{N-P}{2}\right)\left(\mathrm{SSResid}^{\mathrm{I}}\right)^{-\frac{N-P}{2}} .}{\pi^{-\frac{N b-P}{2}} b^{-\frac{N b}{2}}\left|X^{T} X\right|^{-\frac{1}{2}} \Gamma\left(\frac{N b-P}{2}\right)\left(\mathrm{SSResid}^{\mathrm{I}}\right)^{-\frac{N b-P}{2}}} \\
& =\pi^{-\frac{N(1-b)}{2}} b^{\frac{N b}{2}}\left(\mathrm{SSResid}^{\mathrm{I}}\right)^{-\frac{N(1-b)}{2}} \frac{\Gamma\left(\frac{N-P}{2}\right)}{\Gamma\left(\frac{N b-P}{2}\right)} \text {. }
\end{aligned}
$$




\section{Homoscedastic Error Variance, Group-Based Regression Effects}

$$
\begin{aligned}
& P\left(\boldsymbol{Y} \mid m_{s}\right)=\int_{\Theta}\left[(2 \pi)^{-\frac{N}{2}}|\Sigma|^{-\frac{1}{2}} \cdot \exp \left\{-\frac{1}{2}(\boldsymbol{Y}-X \boldsymbol{\beta})^{T} \Sigma^{-1}(\boldsymbol{Y}-X \boldsymbol{\beta})\right\}\right]^{b} P(\boldsymbol{\theta}) d \boldsymbol{\theta} \\
& =\int_{0}^{\infty} \int_{-\infty}^{\infty} \int_{-\infty}^{\infty}(2 \pi)^{-\frac{N b}{2}} \gamma^{\frac{N b}{2}-1} \exp \left\{-b \cdot \frac{1}{2}\left[\boldsymbol{Y}^{T} \Sigma^{-1} \boldsymbol{Y}-2 \boldsymbol{\beta}^{T} X^{T} \Sigma^{-1} \boldsymbol{Y}+\boldsymbol{\beta}^{T} X^{T} \Sigma^{-1} X \boldsymbol{\beta}^{T}\right]\right\} d \boldsymbol{\beta}_{1}^{T} d \boldsymbol{\beta}_{2}^{T} d \gamma \\
& =\int_{0}^{\infty} \int_{-\infty}^{\infty} \int_{-\infty}^{\infty}(2 \pi)^{-\frac{N b}{2}} \gamma^{\frac{N b}{2}-1} \exp \left\{-b \cdot \frac{\gamma}{2}\left[\boldsymbol{\beta}^{T} X^{T} X \boldsymbol{\beta}-2 \boldsymbol{\beta}^{T} X^{T} \boldsymbol{Y}+\boldsymbol{Y}^{T} H \boldsymbol{Y}\right]\right\} \times \\
& \exp \left\{-b \cdot \frac{\gamma}{2}\left[\boldsymbol{Y}^{T} \boldsymbol{Y}-\boldsymbol{Y}^{T} H \boldsymbol{Y}\right]\right\} d \boldsymbol{\beta}_{1} d \boldsymbol{\beta}_{2} d \gamma \\
& =\int_{0}^{\infty}(2 \pi)^{-\frac{N b}{2}} \gamma^{\frac{N b}{2}-1}(2 \pi)^{+\frac{P}{2}}\left|b^{-1} \gamma^{-1}\left(X_{1} X_{1}\right)^{-1}\right|^{+\frac{1}{2}}\left|b^{-1} \gamma^{-1}\left(X_{2} X_{2}\right)^{-1}\right|^{+\frac{1}{2}} \times \\
& \exp \left\{-b \cdot \frac{\gamma}{2}\left[\boldsymbol{Y}_{1}^{T}\left(I-H_{1}\right) \boldsymbol{Y}_{1}+\boldsymbol{Y}_{2}^{T}\left(I-H_{2}\right) \boldsymbol{Y}_{2}\right]\right\} d \gamma \\
& =(2 \pi)^{-\frac{N b-P}{2}} b^{-\frac{P}{2}}\left|X_{1}^{T} X_{1}\right|^{-\frac{1}{2}}\left|X_{2}^{T} X_{2}\right|^{-\frac{1}{2}} \Gamma\left(\frac{N b-P}{2}\right)\left(\frac{b \cdot\left[\operatorname{SSResid}_{1}^{\mathrm{II}}+\operatorname{SSResid}_{2}^{\mathrm{II}}\right]}{2}\right)^{-\frac{N b-P}{2}} \\
& =\pi^{-\frac{N b-P}{2}} b^{-\frac{N b}{2}}\left|X_{1}^{T} X_{1}\right|^{-\frac{1}{2}}\left|X_{2}^{T} X_{2}\right|^{-\frac{1}{2}} \Gamma\left(\frac{N b-P}{2}\right)\left(\operatorname{SSResid}_{1}^{\mathrm{II}}+\operatorname{SSResid}_{2}^{\mathrm{II}}\right)^{-\frac{N b-P}{2}} . \\
& q^{b}\left(\boldsymbol{Y} \mid m_{s}\right)=\frac{\int_{\Theta} P\left(Y \mid m_{s}^{\mathrm{II}}, \boldsymbol{\theta}\right) P(\boldsymbol{\theta}) d \boldsymbol{\theta}}{\int_{\Theta} P^{b}\left(Y \mid m_{s}^{\mathrm{II}}, \boldsymbol{\theta}\right) P(\boldsymbol{\theta}) d \boldsymbol{\theta}} \\
& =\frac{\pi^{-\frac{N-P}{2}} b^{-\frac{N}{2}}\left|X_{1}^{T} X_{1}\right|^{-\frac{1}{2}}\left|X_{2}^{T} X_{2}\right|^{-\frac{1}{2}} \Gamma\left(\frac{N-P}{2}\right)\left(\operatorname{SSResid}_{1}^{\mathrm{II}}+\operatorname{SSResid}_{2}^{\mathrm{II}}\right)^{-\frac{N-P}{2}}}{\pi^{-\frac{N b-P}{2}} b^{-\frac{N b}{2}}\left|X_{1}^{T} X_{1}\right|^{-\frac{1}{2}}\left|X_{2}^{T} X_{2}\right|^{-\frac{1}{2}} \Gamma\left(\frac{N b-P}{2}\right)\left(\operatorname{SSResid}_{1}^{\mathrm{II}}+\operatorname{SSResid}_{2}^{\mathrm{II}}\right)^{-\frac{N b-P}{2}}} \\
& =\pi^{-\frac{N(1-b)}{2}} b^{\frac{N b}{2}}\left(\operatorname{SSResid}_{1}^{\mathrm{II}}+\operatorname{SSResid}_{2}^{\mathrm{II}}\right)^{-\frac{N(1-b)}{2}} \frac{\Gamma\left(\frac{N-P}{2}\right)}{\Gamma\left(\frac{N b-P}{2}\right)} .
\end{aligned}
$$

\section{Heteroscedastic Error Variance}

A Laplace approximation is used to evaluate $\int P(\boldsymbol{Y} \mid \boldsymbol{\varphi}, m) P(\boldsymbol{\varphi}) d \boldsymbol{\varphi}$, parametrized with respect to the $\log$-variances $\lambda_{1}=\log \sigma_{1}^{2}$ and $\lambda_{2}=\log \sigma_{2}^{2}$. Denote $\Lambda$ as the $\log$-variance matrix, 
$J_{\Lambda}$ as the transformation Jacobian, $\left(\lambda_{1}^{\star}, \lambda_{2}^{\star}\right)$ as the mode of the log-marginal distribution, and $\nabla^{\star}$ as the Hessian evaluated at this mode. A subscript of $b$ refers to the same quantities calculated with respect to the fractional exponentiated likelihood. The joint modes $\left(\lambda_{1}^{\star}, \lambda_{2}^{\star}\right)$ and $\left(\lambda_{b_{1}}^{\star}, \lambda_{b_{2}}^{\star}\right)$ are computed using the function optim in R; similarly, the Hessians $\nabla^{\star}$ and $\nabla_{b}^{\star}$ are evaluated at these values using the function hessian in the package numderiv [Gilbert and Varadhan, 2016, R Core Team, 2017].

We first integrate over the regression effects vector $\boldsymbol{\beta}$. Let $H_{\Phi}:=\Phi X\left(X^{T} \Phi X\right)^{-1} X^{T} \Phi$.

$$
\begin{aligned}
& P^{b}\left(\boldsymbol{Y} \mid m_{s}^{\mathrm{III}}, \varphi_{1}, \varphi_{2}\right)=\int_{\Theta}\left[(2 \pi)^{-\frac{N}{2}}|\Phi|^{\frac{1}{2}} \cdot \exp \left\{-\frac{1}{2}(\boldsymbol{Y}-X \boldsymbol{\beta})^{T} \Phi(\boldsymbol{Y}-X \boldsymbol{\beta})\right\}\right]^{b} P(\boldsymbol{\theta}) d \boldsymbol{\theta} \\
& =\int_{-\infty}^{\infty}(2 \pi)^{-\frac{N b}{2}} \varphi_{1}^{\frac{n_{1} b}{2}-1} \varphi_{2}{ }^{\frac{n_{2} b}{2}-1} \exp \left\{-b \cdot \frac{1}{2}\left[\boldsymbol{Y}^{T} \Phi \boldsymbol{Y}-2 \boldsymbol{\beta}^{T} X^{T} \Phi \boldsymbol{Y}+\boldsymbol{\beta}^{T} X^{T} \Phi X \boldsymbol{\beta}^{T}\right]\right\} d \boldsymbol{\beta} \\
& =\int_{-\infty}^{\infty}(2 \pi)^{-\frac{N b}{2}} \varphi^{\frac{n_{1} b}{2}-1} \varphi_{2}^{\frac{n_{2} b}{2}-1} \times \\
& \exp \left\{-b \cdot \frac{1}{2}\left[\boldsymbol{\beta}^{T} X^{T} \Phi X \boldsymbol{\beta}-2 \boldsymbol{\beta}^{T} X^{T} \boldsymbol{Y}+\boldsymbol{Y}^{T} H_{\Phi} \boldsymbol{Y}\right]\right\} \times \\
& \exp \left\{-b \cdot \frac{1}{2}\left[\boldsymbol{Y}^{T} \Phi \boldsymbol{Y}-\boldsymbol{Y}^{T} H_{\Phi} \boldsymbol{Y}\right]\right\} d \boldsymbol{\beta} \\
& =(2 \pi)^{-\frac{N b-P}{2}} \varphi_{1}^{\frac{n_{1} b}{2}-1} \varphi_{2}{ }^{\frac{n_{2} b}{2}-1} b^{-\frac{P}{2}}\left|X^{T} \Phi X\right|^{-\frac{1}{2}} \exp \left\{-b \cdot \frac{1}{2}\left[\boldsymbol{Y}^{T} \Phi \boldsymbol{Y}-\boldsymbol{Y}^{T} H_{\Phi} \boldsymbol{Y}\right]\right\}
\end{aligned}
$$

We reparametrize the precisions of $P^{b}\left(\boldsymbol{Y} \mid m_{s}^{\mathrm{III}}, \varphi_{1}, \varphi_{2}\right)$ and $P\left(\boldsymbol{Y} \mid m_{s}^{\mathrm{III}}, \varphi_{1}, \varphi_{2}\right)$ to log-variances $\lambda_{1}$ and $\lambda_{2}$ to elicit a shape more conducive to the Laplace approximation. For $\lambda_{1}=\ln \varphi_{1}^{-1}$ and $\lambda_{2}=\ln \varphi_{2}^{-1}, P\left(\boldsymbol{Y} \mid m_{s}^{\mathrm{III}}, \lambda_{1}, \lambda_{2}\right)=P\left(\boldsymbol{Y} \mid m_{s}^{\mathrm{III}}, \varphi_{1}, \varphi_{2}\right) \cdot\left|J_{\Lambda}\right|$ where $\frac{\partial \varphi_{1}}{\partial \lambda_{1}}=-\exp \left\{-\lambda_{1}\right\}$ and $\frac{\partial \varphi_{2}}{\partial \lambda_{2}}=-\exp \left\{-\lambda_{2}\right\}$, so $\left|J_{\Lambda}\right|=\exp \left\{-\left(\lambda_{1}+\lambda_{2}\right)\right\}$. Thus the Laplace approximation for the fractional marginal model probability is given by

$$
q^{b}\left(\boldsymbol{Y} \mid m_{s}^{\mathrm{III}}\right) \approx \frac{(2 \pi)\left|-\nabla^{\star}\right|^{-\frac{1}{2}} \cdot P\left(\boldsymbol{Y} \mid m_{s}^{\mathrm{III}}, \varphi_{b_{1}}^{\star}, \varphi_{b_{2}}^{\star}\right)\left|J_{\Lambda}^{\star}\right|}{(2 \pi)\left|-\nabla_{b}^{\star}\right|^{-\frac{1}{2}} \cdot P^{b}\left(\boldsymbol{Y} \mid m_{s}^{\mathrm{III}}, \varphi_{b_{1}}^{\star}, \varphi_{b_{2}}^{\star}\right)\left|J_{b_{\Lambda}}^{\star}\right|} .
$$

Empirical comparisons with cubature-based approximations, in addition to the shapes of the marginal densities $P\left(\boldsymbol{Y} \mid m_{s}^{\mathrm{III}}, \lambda_{1}, \lambda_{2}\right)$ and $P^{b}\left(\boldsymbol{Y} \mid m_{s}^{\mathrm{III}}, \lambda_{1}, \lambda_{2}\right)$, indicate that this Laplace 

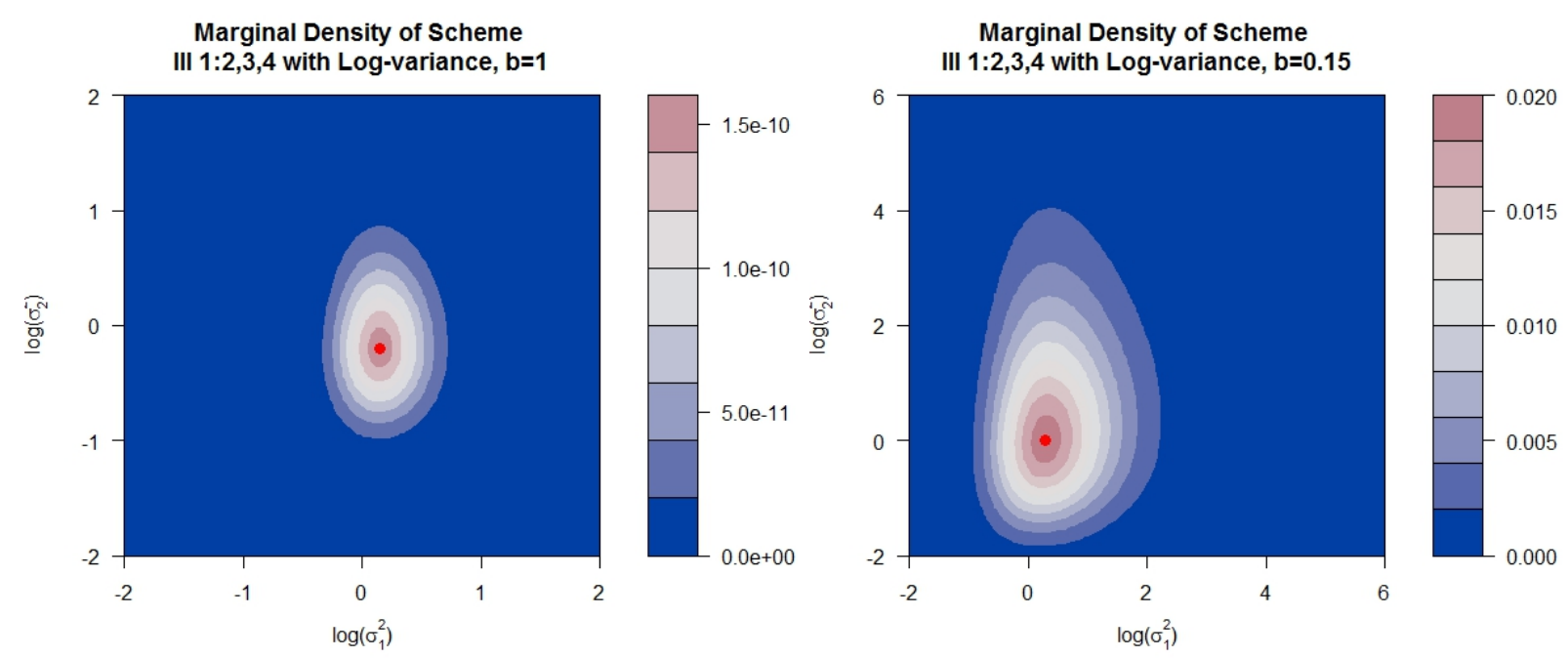

Figure 2.7: The marginal densities $P\left(\boldsymbol{Y} \mid m_{1: 2,3,4}^{\mathrm{II}}, \lambda_{1}, \lambda_{2}\right)$ and $P^{b}\left(\boldsymbol{Y} \mid m_{1: 2,3,4}^{\mathrm{III}}, \lambda_{1}, \lambda_{2}\right)$ for one particular grouping of an ANOVA layout with $K=4$ levels of $n_{k}=10$ observations each show an overall shape conducive to the Laplace approximation in both the raw and exponentiated likelihood cases.

approximation is satisfactory. 
Special Case: Heteroscedastic Error Variance with no Regression Effects Spanning Two Variances

$$
\begin{aligned}
& P\left(\boldsymbol{Y} \mid m_{s}\right)=\int_{\Theta}\left[(2 \pi)^{-\frac{N}{2}}|\Sigma|^{-\frac{1}{2}} \cdot \exp \left\{-\frac{1}{2}(\boldsymbol{Y}-X \boldsymbol{\beta})^{T} \Sigma^{-1}(\boldsymbol{Y}-X \boldsymbol{\beta})\right\}\right]^{b} P(\boldsymbol{\theta}) d \boldsymbol{\theta} \\
& =\int_{0}^{\infty} \int_{0}^{\infty} \int_{-\infty}^{\infty} \int_{-\infty}^{\infty}(2 \pi)^{-\frac{N b}{2}}|\Sigma|^{-\frac{b}{2}} \exp \left\{-b \cdot \frac{1}{2}\left[\boldsymbol{Y}^{T} \Sigma^{-1} \boldsymbol{Y}-2 \boldsymbol{\beta}^{T} X^{T} \Sigma^{-1} \boldsymbol{Y}+\boldsymbol{\beta}^{T} X^{T} \Sigma^{-1} X \boldsymbol{\beta}\right]\right\} \\
& d \boldsymbol{\beta}_{1} d \boldsymbol{\beta}_{2} d \gamma_{1} d \gamma_{2} \\
& =\int_{0}^{\infty} \int_{0}^{\infty} \int_{-\infty}^{\infty} \int_{-\infty}^{\infty}(2 \pi)^{-\frac{N b}{2}} \gamma^{\frac{n_{1} b}{2}-1} \gamma^{\frac{n_{2} b}{2}-1} \exp \left\{-b \cdot \frac{1}{2}\left[\boldsymbol{Y}^{T} \Sigma^{-1} \boldsymbol{Y}-2 \boldsymbol{\beta}^{T} X^{T} \Sigma^{-1} \boldsymbol{Y}+\boldsymbol{\beta}^{T} X^{T} \Sigma^{-1} X \boldsymbol{\beta}\right]\right\} \\
& d \boldsymbol{\beta}_{1} d \boldsymbol{\beta}_{2} d \gamma_{1} d \gamma_{2} \\
& =\int_{0}^{\infty} \int_{0}^{\infty}(2 \pi)^{-\frac{N b}{2}} \gamma^{\frac{n_{1} b}{2}-1} \gamma^{\frac{n_{2} b}{2}-1}(2 \pi)^{+\frac{P}{2}}\left|b^{-1} \gamma^{-1}\left(X_{1}^{T} X_{1}\right)^{-1}\right|^{+\frac{1}{2}}\left|b^{-1} \gamma^{-1}\left(X_{2}^{T} X_{2}\right)^{-1}\right|^{+\frac{1}{2}} \times \\
& \exp \left\{-b \cdot \frac{\gamma}{2}\left[\boldsymbol{Y}_{1}^{T}\left(I-H_{1}\right) \boldsymbol{Y}_{1}+\boldsymbol{Y}_{2}^{T}\left(I-H_{2}\right) \boldsymbol{Y}_{2}\right]\right\} d \gamma_{1} d \gamma_{2} \\
& =(2 \pi)^{-\frac{N b-P}{2}} b^{-\frac{P}{2}}\left|X_{1}^{T} X_{1}\right|^{-\frac{1}{2}}\left|X_{2}^{T} X_{2}\right|^{-\frac{1}{2}} \Gamma\left(\frac{N b-P}{2}\right)\left(\frac{b \cdot\left[\operatorname{SSResid}_{1}^{\mathrm{IV}}+\operatorname{SSResid}_{2}^{\mathrm{IV}}\right]}{2}\right)^{-\frac{N b-P}{2}} \\
& =(2 \pi)^{-\frac{N b-P}{2}} b^{-\frac{P}{2}}\left|X_{1}^{T} X_{1}\right|^{-\frac{1}{2}}\left|X_{2}^{T} X_{2}\right|^{-\frac{1}{2}} \Gamma\left(\frac{n_{1} b-p_{1}}{2}\right) \Gamma\left(\frac{n_{2} b-p_{2}}{2}\right) \times \\
& \left(\frac{b \cdot \operatorname{SSResid}_{1}^{\mathrm{IV}}}{2}\right)^{-\frac{n_{1} b-p_{1}}{2}}\left(\frac{b \cdot \mathrm{SSResid}_{2}^{\mathrm{IV}}}{2}\right)^{-\frac{n_{2} b-p_{2}}{2}} \text {. }
\end{aligned}
$$




$$
\begin{aligned}
q^{b}\left(\boldsymbol{Y} \mid m_{s}\right)= & \frac{\int_{\Theta} P\left(Y \mid m_{s}, \boldsymbol{\theta}\right) P(\boldsymbol{\theta}) d \boldsymbol{\theta}}{\int_{\Theta} P^{b}\left(Y \mid m_{s}^{\mathrm{IV}}, \boldsymbol{\theta}\right) P(\boldsymbol{\theta}) d \boldsymbol{\theta}} \\
= & \frac{(2 \pi)^{-\frac{N-P}{2}}\left|X_{1} X_{1}\right|^{-\frac{1}{2}}\left|X_{2} X_{2}\right|^{-\frac{1}{2}} \Gamma\left(\frac{n_{1}-p_{1}}{2}\right) \Gamma\left(\frac{n_{2}-p_{2}}{2}\right)}{(2 \pi)^{-\frac{N b-P}{2}} b^{-\frac{P}{2}}\left|X_{1} X_{1}\right|^{-\frac{1}{2}}\left|X_{2} X_{2}\right|^{-\frac{1}{2}} \Gamma\left(\frac{n_{1} b-p_{1}}{2}\right) \Gamma\left(\frac{n_{2} b-p_{2}}{2}\right)} \times \\
& \frac{\left(\frac{\operatorname{SSResid}_{1}^{\mathrm{IV}}}{2}\right)^{-\frac{n_{1}-p_{1}}{2}}\left(\frac{\operatorname{SSResid}_{2}^{\mathrm{IV}}}{2}\right)^{-\frac{n_{2}-p_{2}}{2}}}{\left(\frac{b \cdot \mathrm{SSResid}_{1}^{\mathrm{IV}}}{2}\right)^{-\frac{n_{1} b-p_{1}}{2}}\left(\frac{b \cdot \operatorname{SSResid}_{2}^{\mathrm{IV}}}{2}\right)^{-\frac{n_{2} b-p_{2}}{2}}} \\
= & \pi^{-\frac{N(1-b)}{2}} b^{\frac{N b}{2}}\left(\operatorname{SSResid}_{1}^{\mathrm{IV}}\right)^{-\frac{n_{1}(1-b)}{2}}\left(\operatorname{SSResid}_{2}^{\mathrm{IV}}\right)^{-\frac{n_{2}(1-b)}{2}} \frac{\Gamma\left(\frac{n_{1}-p_{1}}{2}\right)}{\Gamma\left(\frac{n_{1} b-p_{1}}{2}\right)} \frac{\Gamma\left(\frac{n_{2}-p_{2}}{2}\right)}{\Gamma\left(\frac{n_{2} b-p_{2}}{2}\right)}
\end{aligned}
$$




\subsubsection{Derivation of Model Probabilities: Mixture $g$ Regression Ef-} fect Priors

$$
\begin{aligned}
& P\left(\boldsymbol{Y}, g \mid m_{s}\right)=\int_{0}^{\infty} \int_{0}^{\infty} \int_{-\infty}^{\infty} \int_{-\infty}^{\infty} P^{b}\left(\boldsymbol{Y} \mid \alpha, \beta, \varphi, m_{s}\right) P(\alpha, \varphi) P(\beta \mid \varphi, g) P(g) d \alpha d \beta d \varphi d g \\
& =\int_{0}^{\infty} \int_{0}^{\infty} \int_{-\infty}^{\infty} \int_{-\infty}^{\infty}(2 \pi)^{-\frac{N b}{2}} \varphi^{\frac{N b}{2}} \exp \left\{-\frac{\varphi b}{2}\left(\boldsymbol{Y}-\mathbf{1}^{T} \alpha-X \boldsymbol{\beta}\right)^{T}\left(\boldsymbol{Y}-\mathbf{1}^{T} \alpha-X \boldsymbol{\beta}\right)\right\} \times \\
& (2 \pi)^{-\frac{P}{2}}\left|g \varphi^{-1}\left(X^{T} X\right)^{-1}\right|^{-\frac{1}{2}} \exp \left\{-\frac{\varphi}{2} \frac{\boldsymbol{\beta}^{T} X^{T} X \boldsymbol{\beta}}{g}\right\} \varphi^{-1} P(g) d \alpha d \boldsymbol{\beta} d \varphi d g \\
& =\int_{0}^{\infty} \int_{0}^{\infty} \int_{-\infty}^{\infty} \int_{-\infty}^{\infty}(2 \pi)^{-\frac{N b+P}{2}} \varphi^{\frac{N b}{2}-1} \times \\
& \exp \left\{-\frac{\varphi}{2}\left(\alpha^{2} b \mathbf{1}^{T} \mathbf{1}-2 \alpha b\left(\mathbf{1}^{T} \boldsymbol{Y}-\mathbf{1}^{T} X \boldsymbol{\beta}\right)+b(\boldsymbol{Y}-X \boldsymbol{\beta}) H_{\mathbf{1}}(\boldsymbol{Y}-X \boldsymbol{\beta})\right)\right\} \times \\
& \left|g \varphi^{-1}\left(X^{T} X\right)^{-1}\right|^{-\frac{1}{2}} \exp \left\{-\frac{\varphi}{2}\left(\boldsymbol{\beta}^{T}\left(\frac{X^{T} X}{g}+b X^{T} X\right) \boldsymbol{\beta}-2 \boldsymbol{\beta}^{T} b X^{T} \boldsymbol{Y}\right)\right\} \times \\
& \exp \left\{-\frac{\varphi}{2}\left(b \boldsymbol{Y}^{T} \boldsymbol{Y}-b(\boldsymbol{Y}-X \boldsymbol{\beta}) H_{1}(\boldsymbol{Y}-X \boldsymbol{\beta})\right)\right\} P(g) d \alpha d \boldsymbol{\beta} d \varphi d g \\
& =\int_{0}^{\infty} \int_{0}^{\infty} \int_{-\infty}^{\infty}(2 \pi)^{-\frac{N b+P-1}{2}} \varphi^{\frac{N b+P-1}{2}-1} b^{-\frac{1}{2}}\left|X^{T} X\right|^{-\frac{1}{2}} N^{-\frac{1}{2}} g^{-\frac{P}{2}} \times \\
& \exp \left\{-\frac{\varphi}{2}\left(\boldsymbol{\beta}^{T}\left(\frac{X^{T} X}{g}+b X^{T} X\right) \boldsymbol{\beta}-2 \boldsymbol{\beta}^{T} b X^{T} \boldsymbol{Y}+\frac{b^{2} g}{1+b g} \boldsymbol{Y}^{T} H_{X} \boldsymbol{Y}\right)\right\} \times \\
& \exp \left\{-\frac{\varphi}{2}\left(b \boldsymbol{Y}^{T} \boldsymbol{Y}-b \boldsymbol{Y}^{T} H_{\mathbf{1}} \boldsymbol{Y}-\frac{b^{2} g}{1+b g} \boldsymbol{Y}^{T} H_{X} \boldsymbol{Y}\right)\right\} P(g) d \boldsymbol{\beta} d \varphi d g \\
& =\int_{0}^{\infty} \int_{0}^{\infty}(2 \pi)^{-\frac{N b-1}{2}} \varphi^{\frac{N b-1}{2}-1} b^{-\frac{1}{2}} N^{-\frac{1}{2}}(1+b g)^{-\frac{P}{2}} \times \\
& \exp \left\{-\frac{\varphi}{2}\left(b \boldsymbol{Y}^{T} \boldsymbol{Y}-b \boldsymbol{Y}^{T} H_{\mathbf{1}} \boldsymbol{Y}-\frac{b^{2} g}{1+b g} \boldsymbol{Y}^{T} H_{X} \boldsymbol{Y}\right)\right\} d \varphi d g \\
& =\int_{0}^{\infty} \frac{\Gamma\left(\frac{N b-1}{2}\right)}{\sqrt{\pi}^{N b-1} \sqrt{N}} b^{-\frac{N b}{2}}(1+b g)^{-\frac{N b-P-1}{2}} \operatorname{SST}^{-\frac{N b-1}{2}}\left[1+b g\left(1-R^{2}\right)\right]^{-\frac{N b-1}{2}} P(g) d g \text {. }
\end{aligned}
$$


After integrating over $\alpha, \boldsymbol{\beta}$, and $\boldsymbol{\varphi}$, we obtain

$$
P\left(\boldsymbol{Y}, g \mid m_{s}^{c}\right)=\int_{0}^{\infty} \frac{\Gamma\left(\frac{N b-1}{2}\right) b^{-\frac{N b}{2}}}{\sqrt{\pi}^{N b-1} \sqrt{N}} \frac{\mathrm{SST}^{-\frac{N b-1}{2}}}{(1+b g)^{\frac{N b-P-1}{2}}}\left[1+b g\left(1-R^{2}\right)\right]^{-\frac{N b-1}{2}} g^{-1.5} \exp \left\{-\frac{N}{2 g}\right\} d g
$$

To execute the Laplace approximation over $g$, we must obtain the mode $g^{\star}=\underset{g}{\operatorname{argmax}} P\left(\boldsymbol{Y}, g \mid m_{s}^{c}\right)$, the root of the equation

$$
-Q b^{2}(P+3) g^{3}+(b(N b-P-4)-2 Q) g^{2}+\left(N b\left(2-R^{2}\right)-3\right) g+N:=0
$$

where $Q=1-R^{2}$. We also require the Hessian evaluated at the mode,

$$
\begin{aligned}
H^{\star} & =\left.\frac{\partial^{2}}{\partial g^{2}}\left[\log \left((1+b g)^{\frac{N b-P-1}{2}}(1+Q b g)^{-\frac{N b-1}{2}} g^{-\frac{3}{2}} \exp \left\{-\frac{N}{2 g}\right\}\right)\right]\right|^{g=g^{\star}} \\
& =\frac{1}{2}\left[\frac{(N b-1) b^{2} Q^{2}}{\left(1+Q b g^{\star}\right)^{2}}-\frac{(N b-P-1) b^{2}}{\left(1+b g^{\star}\right)^{2}}+\frac{3}{\left(g^{\star}\right)^{2}}-\frac{2 N}{\left(g^{\star}\right)^{3}}\right]
\end{aligned}
$$

These expressions are appropriate to use in homoscedastic classes with either global or distinct regression effects; we simply compute the corresponding $R^{2}$ and use the appropriate $P$ based on the model under consideration.

In classes with heteroscedasticity, the integral is intractable over both $\varphi$ and $g$; thus we must employ a three-dimensional Laplace approximation to evaluate this integral. For computational ease and to improve the accuracy of the approximation, we again parametrize with respect to the log-variance; let $\Lambda$ represent the log-variance matrix. Denote $H_{\Lambda}=$ $\Lambda \mathbf{1}\left(\mathbf{1}^{T} \Lambda \mathbf{1}\right)^{-1} \mathbf{1}^{T} \Lambda$; then integrating out the global intercept and regression effects yields an expression for $P^{b}\left(\boldsymbol{Y}, \lambda_{1}, \lambda_{2}, g \mid m_{s}^{c}\right)$ :

$$
\begin{aligned}
& =(2 \pi)^{-\frac{N b+P-1}{2}} \lambda_{1}^{\frac{n_{1} b}{2}-1} \lambda_{2}^{\frac{n_{2} b}{2}-1} g^{-\frac{P}{2}} b^{-\frac{P+1}{2}}\left|X^{T} \Lambda X\right|^{\frac{1}{2}}\left|\mathbf{1}^{T} \Lambda \mathbf{1}\right|^{-\frac{1}{2}}\left|\left(\frac{1+b g}{b g}\right) X^{T} \Lambda X-X^{T} H_{\Lambda} X\right|^{-\frac{1}{2}} \times \\
& J_{\Lambda} \cdot \exp \left\{-\frac{b}{2}\left[\boldsymbol{Y} \Lambda \boldsymbol{Y}-\boldsymbol{Y}^{T} H_{\Lambda} \boldsymbol{Y}-\boldsymbol{Y}^{T}\left(\Lambda-H_{\Lambda}\right)^{T} X\left(\frac{1+b g}{b g} X^{T} \Lambda X-X^{T} H_{\Lambda} X\right) X^{T}\left(\Lambda-H_{\Lambda}\right) \boldsymbol{Y}^{T}\right]\right\}
\end{aligned}
$$

The joint mode $\left(\lambda_{1}^{\star}, \lambda_{2}^{\star}, g^{\star}\right)$ is computed using the function opt im in R; similarly, the Hessian 
is computed at this value using the function hessian in the package numderiv.

\section{Heteroscedastic Zellner-Siow Cauchy Result}

Let $\boldsymbol{\beta} \mid \Phi, g \sim N\left(\mathbf{0}, g\left(X^{T} \Phi X\right)^{-1}\right)$ and $g \sim \operatorname{IG}\left(\frac{1}{2}, \frac{N}{2}\right)$.

$$
\begin{aligned}
& \text { Then } P(\boldsymbol{\beta} \mid g, \Phi)=(2 \pi)^{-\frac{P}{2}}\left|g\left(X^{T} \Phi X\right)^{-1}\right|^{-\frac{1}{2}} \exp \left\{-\frac{1}{2} \boldsymbol{\beta} \frac{X^{T} \Phi X}{g} \boldsymbol{\beta}\right\} \text { and } P(g)=\frac{\left(\frac{N}{2}\right)^{\frac{1}{2}}}{\Gamma\left(\frac{1}{2}\right)} g^{-\frac{3}{2}} \exp \left\{-\frac{N}{2 g}\right\} \text {. } \\
& P(\boldsymbol{\beta} \mid \boldsymbol{\varphi})=\int P(\boldsymbol{\beta} \mid \Phi, g) P(g) d g \\
& =\int(2 \pi)^{-\frac{P}{2}}\left|g\left(X^{T} \Phi X\right)^{-1}\right|^{-\frac{1}{2}} \exp \left\{-\frac{1}{2} \boldsymbol{\beta} \frac{X^{T} \Phi X}{g} \boldsymbol{\beta}\right\} \times \frac{\left(\frac{N}{2}\right)^{\frac{1}{2}}}{\Gamma\left(\frac{1}{2}\right)} g^{-\frac{3}{2}} \exp \left\{-\frac{N}{2 g}\right\} d g \\
& =\int(2 \pi)^{-\frac{P}{2}}\left|X^{T} \Phi X\right|^{\frac{1}{2}}\left(\frac{N}{2}\right)^{\frac{1}{2}} \pi^{-\frac{1}{2}} g^{-\frac{P+3}{2}} \exp \left\{-\frac{1}{2} \boldsymbol{\beta}^{T} \frac{X^{T} \Phi X}{g} \boldsymbol{\beta}-\frac{1}{2} \cdot \frac{N}{g}\right\} d g \\
& =\int(2 \pi)^{-\frac{P}{2}}\left|X^{T} \Phi X\right|^{\frac{1}{2}} N^{\frac{1}{2}} 2^{-\frac{1}{2}} \pi^{-\frac{1}{2}} \times g^{-\frac{P+1}{2}-1} \exp \left\{-\frac{\boldsymbol{\beta}^{T} X^{T} \Phi X \boldsymbol{\beta}+N / 2}{g}\right\} d g \\
& =2^{-\frac{P+1}{2}} \pi^{-\frac{P+1}{2}}\left|X^{T} \Phi X\right|^{\frac{1}{2}} N^{\frac{1}{2}}\left[\left(\boldsymbol{\beta}^{T} X^{T} \Phi X \boldsymbol{\beta}+N\right)^{\frac{P+1}{2}} 2^{-\frac{P+1}{2}} \Gamma\left(\frac{P+1}{2}\right)\right]^{-1} \\
& =\pi^{-\frac{P+1}{2}}\left|X^{T} \Phi X\right|^{\frac{1}{2}} N^{\frac{1}{2}}\left(\boldsymbol{\beta}^{T} X^{T} \Phi X \boldsymbol{\beta}+N\right)^{-\frac{P+1}{2}} \Gamma\left(\frac{P+1}{2}\right)^{-1} \\
& =\pi^{-\frac{P+1}{2}}\left|X^{T} \Phi X\right|^{\frac{1}{2}} N^{\frac{1}{2}}\left(\frac{\boldsymbol{\beta}^{T} X^{T} \Phi X \boldsymbol{\beta}+N}{N}\right)^{-\frac{P+1}{2}} N^{-\frac{P+1}{2}} \Gamma\left(\frac{P+1}{2}\right)^{-1} \\
& =\pi^{-\frac{P+1}{2}}\left|X^{T} \Phi X\right|^{\frac{1}{2}} N^{-\frac{P}{2}}\left(1+\boldsymbol{\beta}^{T} \frac{X^{T} \Phi X}{N} \boldsymbol{\beta}\right)^{-\frac{P+1}{2}} \Gamma\left(\frac{P+1}{2}\right)^{-1} \\
& =\pi^{-\frac{p+1}{2}} \Gamma\left(\frac{P+1}{2}\right)^{-1} \times\left|\frac{X^{T} \Phi X}{N}\right|^{\frac{1}{2}}\left(1+\boldsymbol{\beta}^{T} \frac{X^{T} \Phi X}{N} \boldsymbol{\beta}\right)^{-\frac{P+1}{2}} \\
& \sim \operatorname{MVCauchy}_{P}\left(\text { location }=0, \text { scale }=\left(\frac{X^{T} \Phi X}{N}\right)^{-1}\right) \text {. }
\end{aligned}
$$

\subsubsection{Supplemental ANCOVA Simulation Study}

We let $n_{k}:=10$ for each level of the SLGF. The parameter settings are provided in Table 2.1 . 

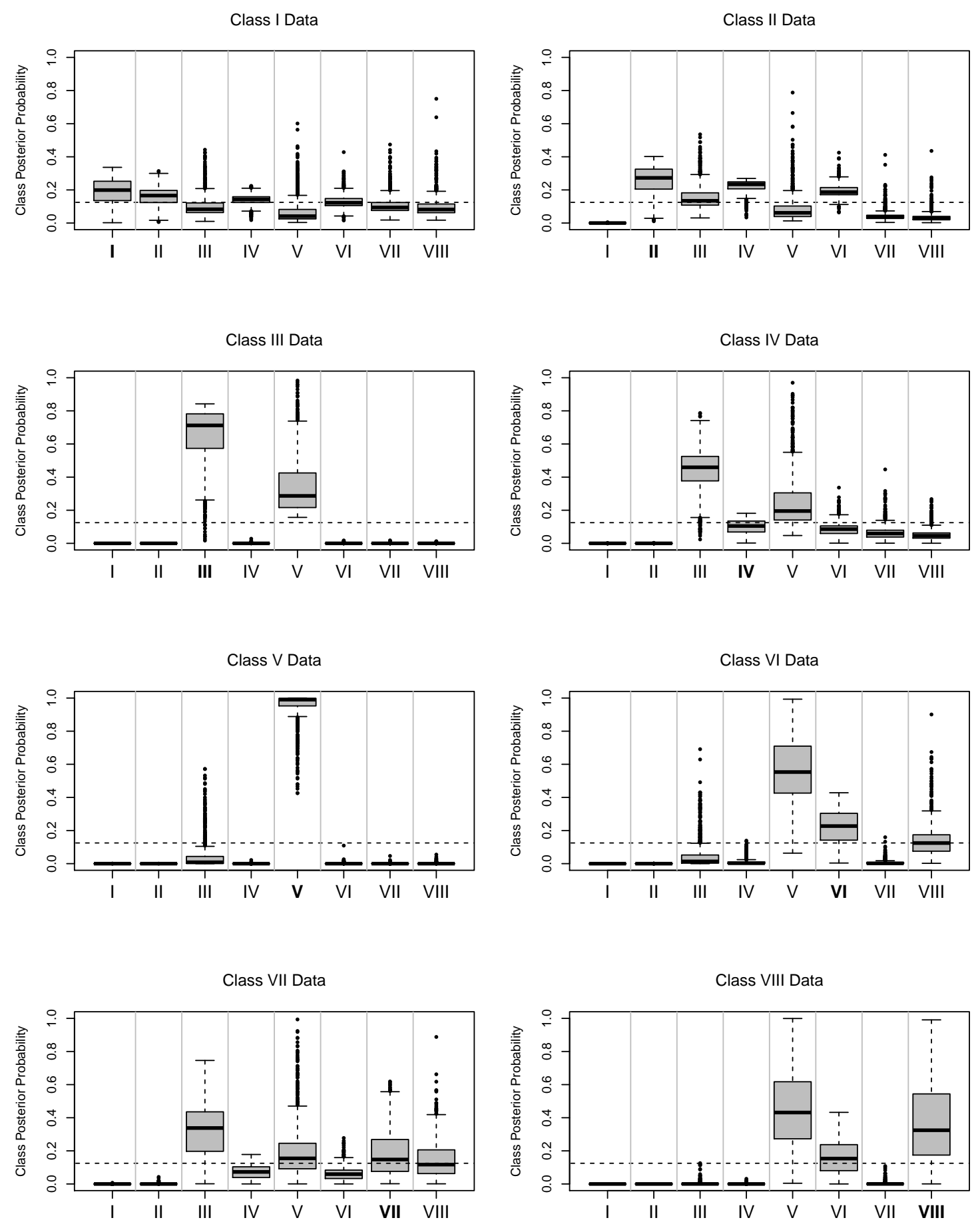

Figure 2.8: Posterior probabilities (y-axis) by class based on 1000 Monte Carlo data sets with $K=4$ levels of the categorical predictor, each with 10 observations, for a total of $N=40$ observations. The true model class is emphasized in bold on the $x$-axis. The dashed line indicates the prior by model class. 


\begin{tabular}{|c|c|}
\hline Class & Parameters \\
\hline I (Additive Model) & $\boldsymbol{\nu} \in\{1,2,3,4,5,7,8,9,10\}, \boldsymbol{\tau} \in\{1,2,3,4,5\}, \sigma^{2}=1$ \\
\hline II (Group-by-Column & $\boldsymbol{\nu} \in\{1,2,3,4,5,7,8,9,10\}, \boldsymbol{\tau}_{1} \in\{1.0,1.5,2.0,2.5,3.0\}$, \\
Interaction) & $\boldsymbol{\tau}_{2} \in\{3.0,2.5,2.0,1.5,1.0\}, \sigma^{2}=1$ \\
\hline III (Heteroscedastic & $\boldsymbol{\nu} \in\{1,2,3,4,5,7,8,9,10\}, \boldsymbol{\tau} \in\{1,2,3,4,5\}$, \\
Additive) & $\sigma_{1}^{2}=1.0, \sigma_{2}^{2}=0.25$ \\
\hline IV (Heteroscedastic Group- & $\boldsymbol{\nu} \in\{1,2,3,4,5,7,8,9,10\}, \boldsymbol{\tau}_{1} \in\{1.0,1.5,2.0,2.5,3.0\}$, \\
by-Column Interaction) & $\boldsymbol{\tau}_{2} \in\{3.0,2.5,2.0,1.5,1.0\}, \sigma_{1}^{2}=1.0, \sigma_{2}^{2}=0.25$ \\
\hline
\end{tabular}

Table 2.3: Settings for the four model classes in the $10 \times 5$ two-way layout simulation study with smaller effect size.

\begin{tabular}{|c|c|}
\hline Class & Parameters \\
\hline I (Additive Model) & $\boldsymbol{\nu} \in\{1,1.5,2,2.5,3\}, \boldsymbol{\tau} \in\{1,2,3,4,5\}, \sigma^{2}=1$ \\
\hline II (Group-by-Column & $\boldsymbol{\nu} \in\{1,2,3,4,5\}, \boldsymbol{\tau}_{1} \in\{1.0,1.8,2.6,3.4,4.2\}$, \\
Interaction) & $\boldsymbol{\tau}_{2} \in\{4.2,3.4,2.6,1.8,1.0\}, \sigma^{2}=1$ \\
\hline III (Heteroscedastic & $\boldsymbol{\nu} \in\{1,2,3,4,5\}, \boldsymbol{\tau} \in\{1,2,3,4,5\}$, \\
Additive) & $\sigma_{1}^{2}=1.0, \sigma_{2}^{2}=0.25$ \\
\hline IV (Heteroscedastic Group- & $\boldsymbol{\nu} \in\{1,2,3,4,5\}, \boldsymbol{\tau}_{1} \in\{1.0,1.8,2.6,3.4,4.2\}$, \\
by-Column Interaction) & $\boldsymbol{\tau}_{2} \in\{4.2,3.4,2.6,1.8,1.0\}, \sigma_{1}^{2}=1.0, \sigma_{2}^{2}=0.25$ \\
\hline
\end{tabular}

Table 2.4: Settings for the four model classes in the two-way layout simulation study with $5 \times 5$ layouts with larger effect size.

\subsubsection{Supplemental Two-way Layout Simulation Study}

We provide three additional simulation studies in the twoway layout scenario: $10 \times 5$ layouts with a smaller effect size than the study provided in Section 2.4 .2 , as well as $5 \times 5$ studies with larger and smaller effect sizes. 

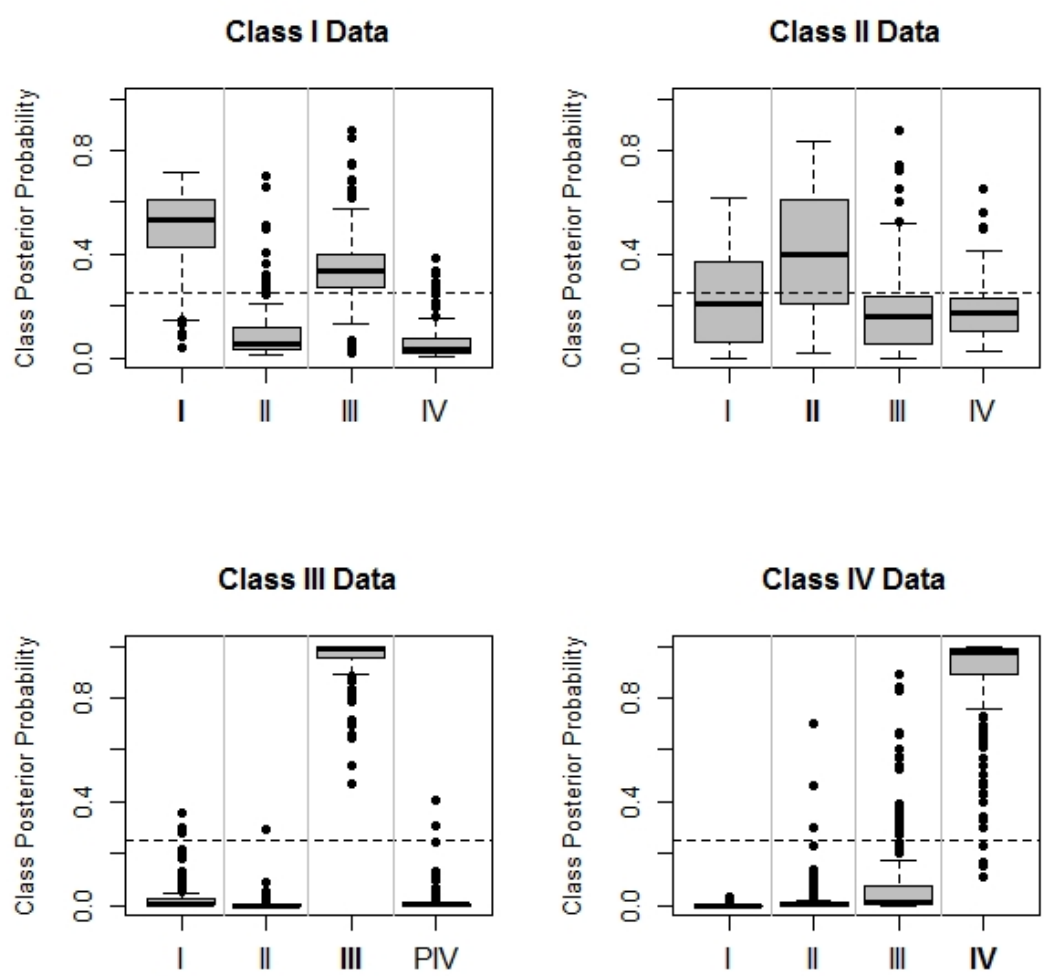

Figure 2.9: Posterior probabilities ( $y$-axis) by class based on 1000 Monte Carlo $10 \times 5$ layouts with smaller effect size. The true model class is emphasized in bold on the $x$-axis. The dashed line indicates the prior by model class.

\begin{tabular}{|c|c|}
\hline Class & Parameters \\
\hline I (Additive Model) & $\boldsymbol{\nu} \in\{1,2,3,4,5\}, \boldsymbol{\tau} \in\{1,2,3,4,5\}, \sigma^{2}=1$ \\
\hline II (Group-by-Column & $\boldsymbol{\nu} \in\{1,2,3,4,5\}, \boldsymbol{\tau}_{1} \in\{1.0,1.5,2.0,2.5,3.0\}$, \\
Interaction) & $\boldsymbol{\tau}_{2} \in\{3.0,2.5,2.0,1.5,1.0\}, \sigma^{2}=1$ \\
\hline III (Heteroscedastic & $\boldsymbol{\nu} \in\{1,2,3,4,5\}, \boldsymbol{\tau} \in\{1,2,3,4,5\}$, \\
Additive) & $\sigma_{1}^{2}=1.0, \sigma_{2}^{2}=0.25$ \\
\hline IV (Heteroscedastic Group- & $\boldsymbol{\nu} \in\{1,2,3,4,5\}, \boldsymbol{\tau}_{1} \in\{1.0,1.5,2.0,2.5,3.0\}$, \\
by-Column Interaction) & $\boldsymbol{\tau}_{2} \in\{3.0,2.5,2.0,1.5,1.0\}, \sigma_{1}^{2}=1.0, \sigma_{2}^{2}=0.25$ \\
\hline
\end{tabular}

Table 2.5: Settings for the four model classes in the two-way layout simulation study with $5 \times 5$ layouts with smaller effect size. 

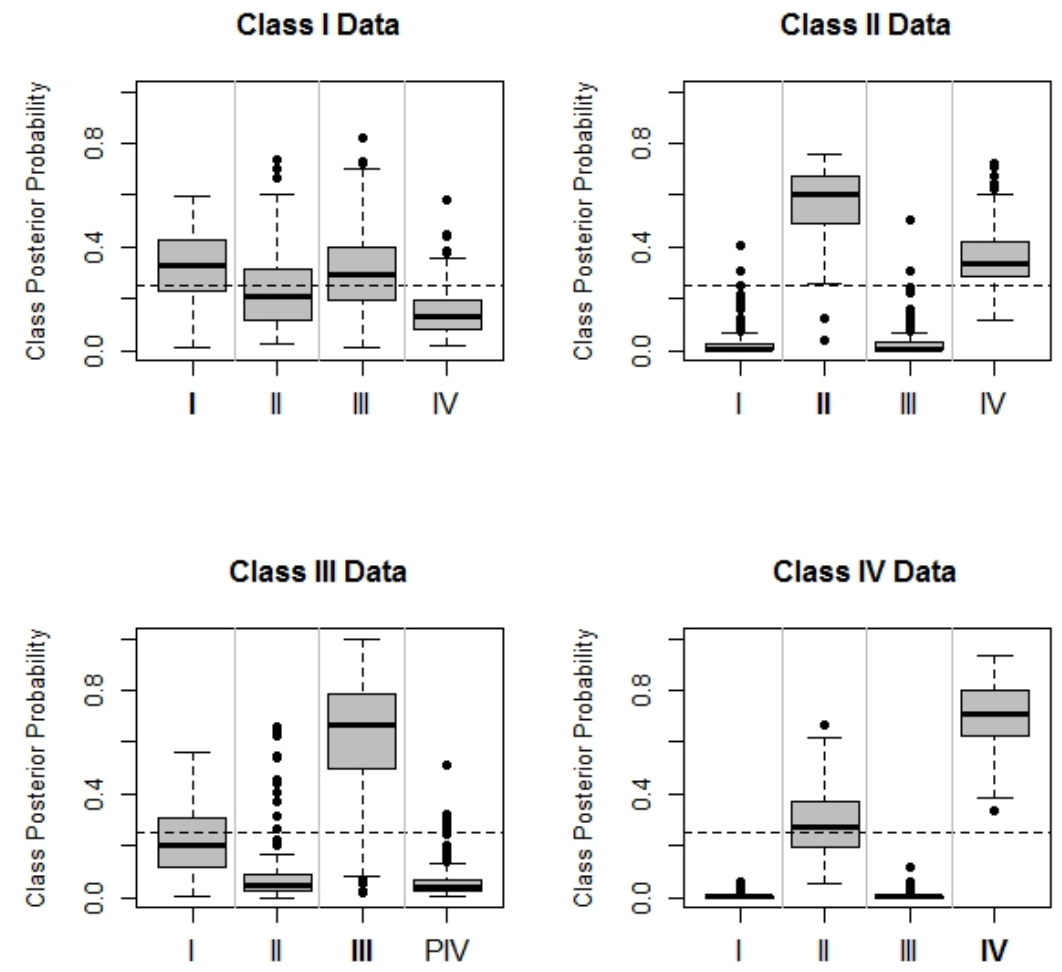

Figure 2.10: Posterior probabilities ( $y$-axis) by class based on 1000 Monte Carlo $5 \times 5$ layouts with larger effect size. The true model class is emphasized in bold on the $x$-axis. The dashed line indicates the prior by model class. 

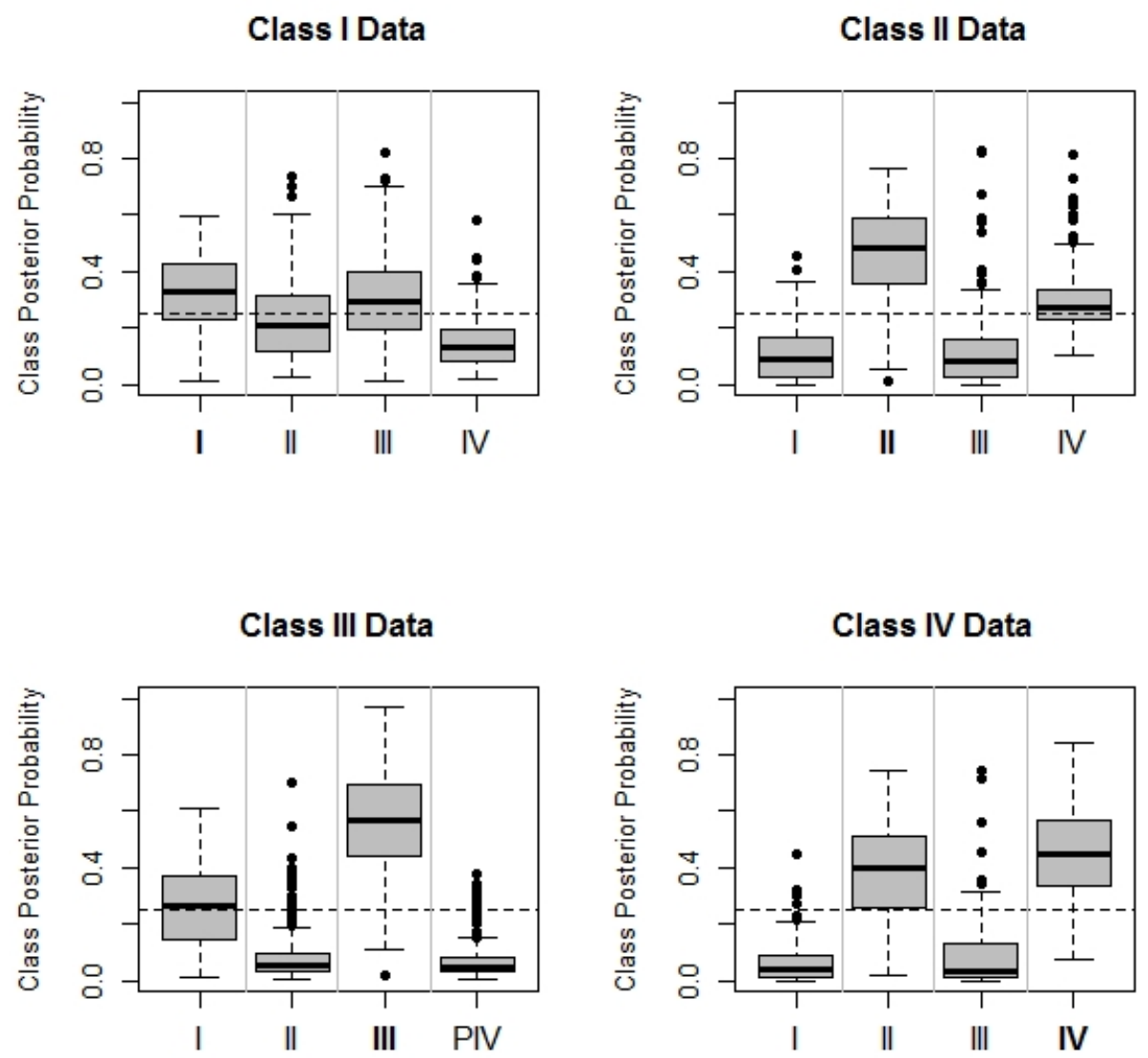

Figure 2.11: Posterior probabilities ( $y$-axis) by class based on 1000 Monte Carlo $5 \times 5$ layouts with smaller effect size. The true model class is emphasized in bold on the $x$-axis. The dashed line indicates the prior by model class. 


\section{Chapter 3}

\section{Bayesian Model Selection with}

\section{Group-Based Regression Effects and}

\section{Heteroscedasticity using the $\mathbf{R}$}

\section{Package slgf}

Linear models are flexible and among the most frequently implemented statistical methods. However, their performance can suffer when assumptions are violated. We focus on the scenario in which the levels of a categorical predictor exhibit two latent groups, leading to group-based regression effects and/or heteroscedastic error variance. First we review the SLGF (suspected latent grouping factor) method. Next, using both observational and experimental data, we illustrate the usage of the $\mathrm{R}$ package slgf in the context of several common linear model layouts: one-way analysis of variance (ANOVA), analysis of covariance (ANCOVA), a two-way replicated layout, a two-way unreplicated layout, and a balanced incomplete block design (BIBD). We have selected data that reveal the shortcomings of classical analyses to emphasize the advantage our method can provide when a latent grouping structure underlies the data. 


\subsection{Introduction}

Linear models with categorical predictors are pervasive in the social and natural sciences. Many well-established classical and Bayesian methods exist to analyze such models, but conventional approaches may often rely on assumptions that are restrictive and fail to account for more subtle latent structures within the data. Several examples of such datasets can be found in Franck and Osborne [2016], Franck [2018], Kharrati-Kopaei and Sadooghi-Alvandi [2007], and Metzger and Franck [2019]. Consider Figure 3.1, which illustrate three of the five datasets considered herein. In each case, upon inspecting the plot, the researcher might suspect that there is a latent grouping structure (indicated by color-coding) where groups are formed by partitioning the levels of a factor; we call this the suspected latent grouping factor, or SLGF. The left panel represents a one-way analysis of variance (ANOVA) study where a continuous measurement of olfactory acuity ( $y$-axis) is modeled as a function of age, where age is represented in five categories ( $x$-axis) [O'Brien and Heft, 1995]. We suspect that the levels 1, 2, and 3 of the slgf, age, have distinct means and error variances from levels 4 and 5. The center panel shows a BIBD, where abrasion from a road test was measured on four tires (blocks). Four treatments (compounds used to manufacture the tires) were analyzed, but due to manufacturing limitations each tire was made from sections of three compounds each. Compounds A and B appear to behave similarly to one another, as do C and D. Finally, the right panel shows the example described by Meek and Ozgur [1991], where the torque required to tighten a locknut was measured as a function of a plating process and a threading technique. The plating processes analyzed included treatments with cadmium and wax, heat treating, and phosphate and oil, denoted CW, HT, and PO, respectively. The threading techniques studied include bolt and mandrel, the types of fixture on which each locknut was affixed to conduct the test. We suspect that observations measured on a bolt may have a higher error variance than those from the mandrel. 

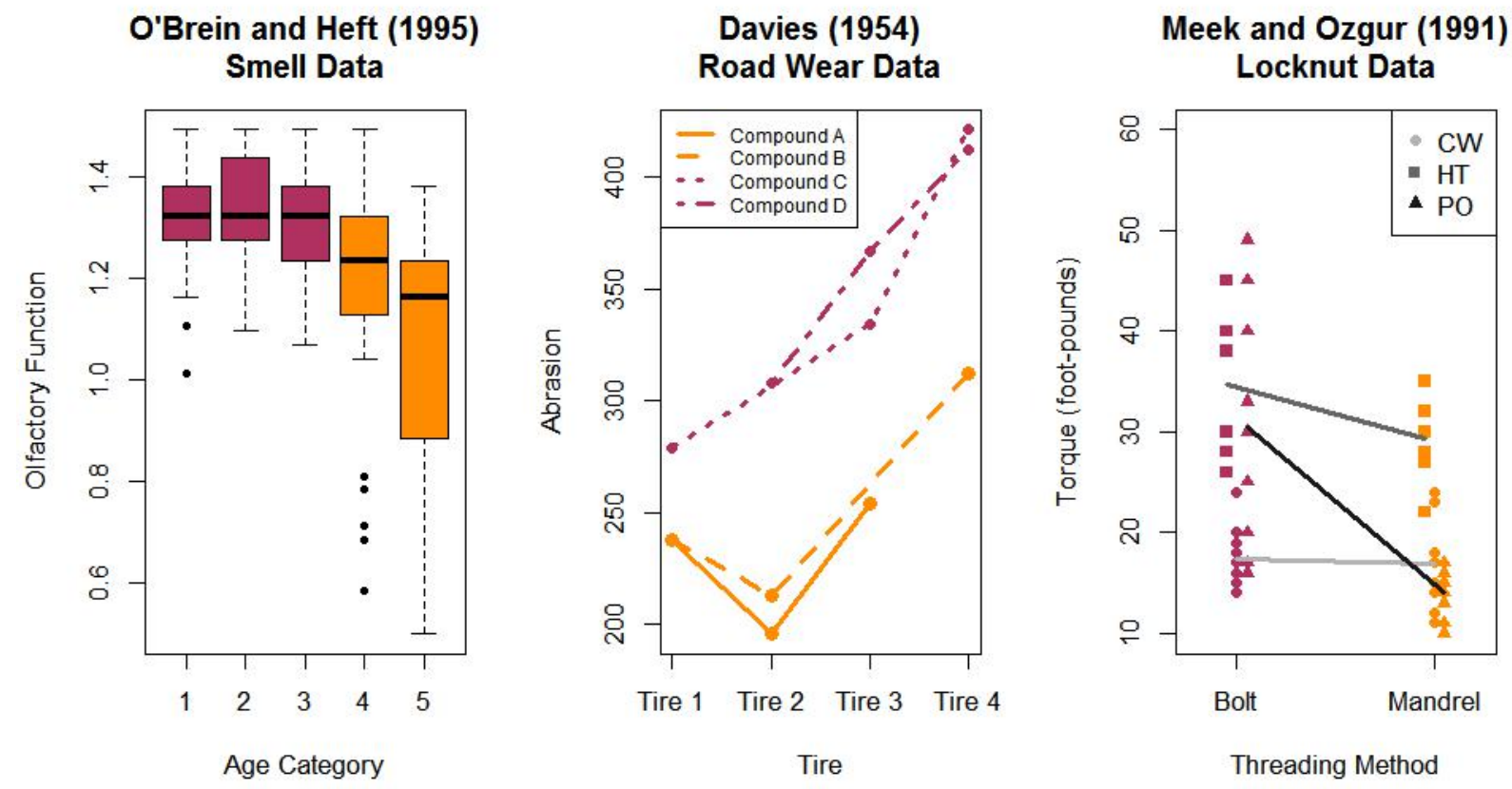

Figure 3.1: Data from O'Brien and Heft [1995] (left), Davies [1954] (center), and Meek and Ozgur [1991] (right). These data sets appear to have a latent grouping structure, color-coded for emphasis. The left panel appears to show two groups with both distinct means and error variances; the center panel shows two groups with distinct regression effects; and the right panel shows group-based error variances, and group-based interactions may also be present. 


\subsubsection{Model Classes and Grouping Schemes}

We first describe the model classes associated with the SLGF methodology; for a thorough review see Metzger and Franck [2019]. Note that the color-coding in Figure 3.1 is based on subjective appearance. In the absence of prior information regarding grouping structures, we consider all possible ways to partition the levels of the SLGF We denote $\mathcal{S}$ as the set of all such possible grouping schemes. The function $\mathrm{lm} . \mathrm{slgf}$ automatically considers all possible grouping schemes based on a user-chosen SLGF, where the user can also specify the minimum number of levels of the SLGF that may comprise an individual group. Notationally, we denote the grouping schemes of Figure 3.1 as 1,2,3:4,5 and A,B:C,D and bolt:mandrel respectively. The number of possible grouping schemes grows exponentially with the number of levels of the SLGF, so slgf is recommended for use with categorical predictors with 10 or fewer levels.

Additionally, our suspicions in Figure 3.1 from left to right of group-based means and variances, group-based regression effects, and group-based variances are also nothing but subjective guesses at this point. We must also account for all such user-specified model structures in addition to the combinatorically possible grouping schemes. We thus introduce the concept of a model class, which summarizes the model effects along with its error variance structure (either homoscedastic or heteroscedatic). We illustrate this idea with the roadwear data set in the center panel of Figure 3.1:

\begin{tabular}{lrrr}
\multicolumn{4}{l}{ head(roadwear) } \\
abrasion & compound & tire \\
1 & 238 & A & 1 \\
2 & 238 & B & 1 \\
3 & 279 & C & 1 \\
4 & 196 & A & 2 \\
5 & 213 & B & 2 \\
6 & 308 & D & 2
\end{tabular}


The column abrasion denotes the continuous response, compound the treatment factor, and tire the blocking factor. We consider compound as the SLGF. Thus we might reasonably consider many models; three non-exhaustive examples are provided here:

1. abrasion compound+tire, where the compound and tire effects each have three degrees of freedom. This simple, standard model does not rely on the SLGF methodology, and its inclusion as a candidate helps assess the plausibility that a grouping structure actually underlies the data.

2. abrasion $\sim$ group+tire, where the tire effect again has three degrees of freedom, but the group effect contracts four levels of compound into two groups so the group effect has only one degree of freedom, thus reducing the dimensionality of the model.

3. abrasion group, where the only effect estimated is a one parameter group effect.

These models are just three possible parametrizations, and slgf allows the user to tailor the set of candidate models to subject-specific considerations. When the user includes the string group in a model specification, the SLGF is automatically considered in the context of a group-based effect. With each such specification, the user must also specify whether there is also latent group-based heteroscedasticity; when a 1 is indicated in the corresponding argument of het, group-based variances will automatically be considered. It is possible to consider group-based variances without group-based regression effects if the group term does not appear in the corresponding usermodel. Such a model specification, along with either a single error variance or group-based error variances, comprise a model class; see Section 3.4 for more detail.

Note that some model classes may contain one model only. For example, when the first example given above is considered in the model space, there is only possible candidate model. On the other hand, example 2 contains a group effect, and there are six possible grouping schemes by compound, so this model class contains six models. If example 1 is considered with group-based variances, there are still three possible grouping schemes for the variances but 
only one possible regression effect specification, so this class would also contain six models. See Metzger and Franck [2019] for a more thorough description of grouping variables and schemes.

\subsubsection{Classical Approach}

Let us narrow our focus to the leftmost panel, the olfactory data set. A researcher's first inclination for analysis might consist of the following steps:

1. Choose a signifcance level $\alpha$;

2. For the age-level means $\left\{\mu_{i}\right\}_{i=1}^{5}$, conduct an $F$-test to compare the null hypothesis, $H_{0}: \mu_{1}=\mu_{2}=\mu_{3}=\mu_{4}=\mu_{5}$ vs. $H_{A}$ : at least one mean is different;

3. If we reject the null hypothesis, conduct a post-hoc analysis to compare the distinct means of each age category.

In $\mathrm{R}$, this analysis might is usually conducted by fitting a null model with a single mean effect, an alternative model with an age category effect with four degrees of freedom, and comparing the two via the anova function: We note that the $p$-value of the ANOVA $F$-test is approximately $1.3947 \times 10^{-11}$, and thus conclude that there are distinct means for any reasonable significance level $\alpha$. We then might perform a post-hoc analysis, such as the Tukey honest significant difference test [Tukey, 1949]:

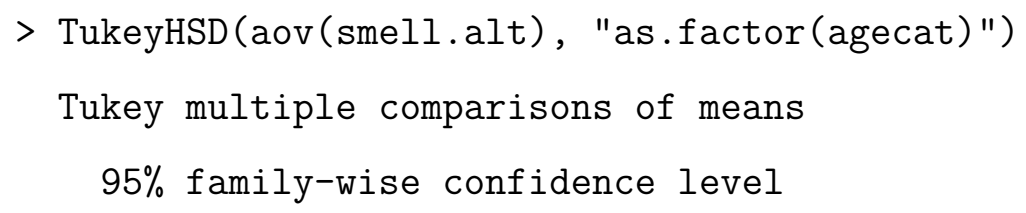

Fit: aov (formula $=$ smell.alt) 


$$
\begin{array}{rrrrr}
\text { \$`as.factor (agecat) } & \text { upr } & \text { p adj } \\
\text { diff } & \text { lwr } & 0.143127531 & 0.9610275 \\
2-1 & 0.02824415 & -0.08663923 & 0.123558306 & 0.9994692 \\
3-1 & -0.01075188 & -0.14506207 & 0.123 \\
4-1 & -0.11580171 & -0.22577846 & -0.005824966 & 0.0335696 \\
5-1 & -0.25727569 & -0.36786495 & -0.146686433 & 0.0000000 \\
3-2 & -0.03899603 & -0.17462764 & 0.096635579 & 0.9325096 \\
4-2 & -0.14404587 & -0.25563257 & -0.032459160 & 0.0043323 \\
5-2 & -0.28551984 & -0.39771027 & -0.173329416 & 0.0000000 \\
4-3 & -0.10504983 & -0.23655127 & 0.026451604 & 0.1838271 \\
5-3 & -0.24652381 & -0.37853793 & -0.114509694 & 0.0000070 \\
5-4 & -0.14147398 & -0.24863454 & -0.034313415 & 0.0032756
\end{array}
$$

Note this method computes $\frac{\left(\begin{array}{l}5 \\ 2\end{array}\right)}{2}=10 p$-values for pairwise difference comparisons, concluding, for instance, that the means of levels 1 and 2, 1 and 3, and 2 and 3 are statistically equivalent. On the other hand, levels 1 and 5, among many others, have statistically distinct means. The comparison between levels 4 and 1 has a $p$-value significant at $\alpha=0.05$, but not $\alpha=0.01$. And perhaps most importantly, this method does not account for the apparent group-based heteroscedasticity.

Our proposed method and the slgf package address these concerns in a user-friendly manner. The user can specify the model classes they wish to consider using familiar $\mathrm{R}$ syntax, and posterior model probabilities are automatically computed for each model class and grouping scheme. This can summarize both the effects that should be included in the model, and the latent grouping structure underlying the SLGF, if it is indeed present.

The remainder of this chapter is organized as follows: Section 2 provides the mathematical background of the SLGF approach; Section 3 outlines the functions that comprise the slgf package; Section 4 provides detailed examples demonstrating the functionality of slgf; and Section 5 provides discussion and future avenues to improve the package. 


\subsection{Mathematical Details}

\subsubsection{Model Specification}

We next briefly review a model framework flexible enough to account for all possible model classes, employing the notation of Metzger and Franck [2019]. We begin with the model

$$
\boldsymbol{Y}=X \boldsymbol{\beta}+\boldsymbol{\varepsilon}
$$

where $\boldsymbol{Y}$ is an $N \times 1$ vector of continuous observations; $X$ is an $N \times P$ design matrix; $\boldsymbol{\beta}$ is a $P \times 1$ vector of regression effects; and $\varepsilon$ is an $N \times 1$ residual vector where $\varepsilon \sim N(\mathbf{0}, \Sigma)$. We must augment our notation to account for the full SLGF with $K$ degrees of freedom or a 2-degree of freedom group effect; interactions with the SLGF or group effect; other effects of interest unrelated to the SLGF; and potential group-based heteroscedasticity. Thus we let $X=\left(\mathbf{1}^{T}|W| V \mid U\right)$ and $\boldsymbol{\beta}=(\alpha, \boldsymbol{\nu}, \boldsymbol{\tau}, \boldsymbol{\rho})$ to obtain

$$
\boldsymbol{Y}=\mathbf{1}^{T} \alpha+W \boldsymbol{\nu}+V \boldsymbol{\tau}+U \boldsymbol{\rho}+\boldsymbol{\varepsilon}
$$

where $\mathbf{1}^{T}$ is an $N \times 1$ vector of $1 \mathrm{~s} ; \alpha$ is a scalar intercept common to all models; $\boldsymbol{\nu}$ is the $K$-dimensional vector of SLGF effects; $W$ is the corresponding $K \times N$ design matrix; $\boldsymbol{\tau}$ is the $J$-dimensional vector of additional effects; $V$ is the corresponding $J \times N$ design matrix; $\boldsymbol{\rho}$ is the $L$-dimensional vector of SLGF-interaction effects; and $U$ is the corresponding $L \times N$ design matrix.

Not all linear models will incorporate each term; for example, the ANOVA layout in the left panel of Figure 1 contains only a single categorical predictor so $\tau:=\mathbf{0}$ and $\rho:=\mathbf{0}$. When the SLGF is modeled as a group effect, denote this effect as $\tilde{\boldsymbol{\nu}}$; when there is an interaction involving the group effect, denote it as $\tilde{\boldsymbol{\rho}}$.

In heteroscedastic contexts, let $\sigma_{1}^{2}$ and $\sigma_{2}^{2}$ represent the error variances of groups 1 and 
2 , respectively. Let $\tilde{\Sigma}$ denote the covariance matrix where the $i$ th diagonal element is $\sigma_{1}^{2}$ if $y_{i}$ belongs to group 1 , or $\sigma_{2}^{2}$ if $y_{i}$ belongs to group 2 .

\subsubsection{Parameter Priors}

For the usual case where prior information is unavailable, we prefer noninformative priors on the regression effects and error variance(s); for the regression effects, this is a flat prior. For homoscedastic models,

$$
P\left(\boldsymbol{\beta}, \sigma^{2} \mid m\right) \propto \frac{1}{\sigma^{2}}
$$

and for a heteroscedastic models

$$
P\left(\boldsymbol{\beta}, \sigma_{1}^{2}, \sigma_{2}^{2} \mid m\right) \propto \frac{1}{\sigma_{1}^{2} \cdot \sigma_{2}^{2}}
$$

However, in contexts with limited data, such as the two-way unreplicated layout and BIBD illustrated in Sections 4.3 and 4.4, respectively, we employ the Zellner-Siow mixture $g$-prior [Zellner and Siow, 1980, Zellner, 1986, Liang et al., 2008], which reduces the minimal training sample size necessary for the computation of the fractional Bayes factor (see Section 3.2.3 for further detail). For homoscedastic models,

$$
P\left(\alpha, \sigma^{2} \mid m\right) \propto \frac{1}{\sigma^{2}} \text { and } \boldsymbol{\beta}_{-\alpha} \mid \Sigma, g, m \sim N\left(\mathbf{0}, g\left(X^{T} \Sigma X\right)^{-1}\right)
$$

for heteroscedastic models,

$$
P\left(\alpha, \sigma_{1}^{2}, \sigma_{2}^{2} \mid m\right) \propto \frac{1}{\sigma_{1}^{2} \cdot \sigma_{2}^{2}} \text { and } \boldsymbol{\beta}_{-\alpha} \mid \tilde{\Sigma}, g, m \sim N\left(\mathbf{0}, g\left(X^{T} \tilde{\Sigma} X\right)^{-1}\right)
$$

and in both cases,

$$
g \sim \operatorname{IG}\left(\frac{1}{2}, \frac{N}{2}\right)
$$




\subsubsection{Fractional Bayes Factors and Posterior Model Probabilities}

Note that if we were to form a standard Bayes factor comparing a homoscedastic and heteroscedastic model, the constants associated with the noninformative priors would not cancel one another and the Bayes factor would be defined only up to an unspecified constant. Thus we invoke a fractional Bayes factor approach to compute well-defined posterior model probabilities for each model; for a thorough review see O'Hagan [1995], and see Metzger and Franck [2019] for a discussion of fractional Bayes factors in the context of this problem.

Let $\mathcal{M}$ represent the full set of models under consideration, representing all classes and grouping schemes of interest. Denote $\boldsymbol{\theta}$ as the full set of unknown parameters associated with a model $m \in \mathcal{M}$ and $\pi(\boldsymbol{\theta})$ as the joint prior on these parameters. A fractional Bayes is a ratio of two fractional marginal model probabilites, where a fractional marginal likelihood is defined as

$$
q^{b}(\boldsymbol{Y} \mid \boldsymbol{\theta})=\frac{\int P(\boldsymbol{Y} \mid \boldsymbol{\theta}, m) \pi(\boldsymbol{\theta}) d \boldsymbol{\theta}}{\int P(\boldsymbol{Y} \mid \boldsymbol{\theta}, m)^{b} \pi(\boldsymbol{\theta}) d \boldsymbol{\theta}}
$$

for a some fractional exponent $b$. Although O'Hagan [1995] provides several recommendations for choice of $b$, slgf exclusively implements $b=\frac{m_{0}}{N}$ where $m_{0}$ is the minimal training sample size required for $P(\boldsymbol{Y} \mid m)$ to be a proper distribution. Thus we must compute the integrals $\int P(\boldsymbol{Y} \mid \boldsymbol{\theta}, m) \pi(\boldsymbol{\theta}) d \boldsymbol{\theta}$ and $\int P(\boldsymbol{Y} \mid \boldsymbol{\theta}, m)^{b} \pi(\boldsymbol{\theta}) d \boldsymbol{\theta}$ for all $m \in \mathcal{M}$. In the case of nonin-

formative regression priors, $\boldsymbol{\beta}$ is integrated analytically, and $\sigma^{2}$ or $\sigma_{1}^{2}, \sigma_{2}^{2}$ are integrated using a Laplace approximation after a log-variance transformation. In the Zellner-Siow mixture $g$ prior case, $\alpha$ and $\boldsymbol{\beta}_{-\alpha}$ are integrated analytically, and $\sigma^{2}$ or $\sigma_{1}^{2}, \sigma_{2}^{2}$ and $g$ are again integrated using a Laplace approximation with a log-variance transformation. Let $\tilde{\boldsymbol{\theta}}$ represent the set of unknown parameters after the regression effects $\boldsymbol{\beta}$ have been integrated out. Then for dimensionality $d=2$ in the noninformative prior case and $d=3$ in the Zellner-Siow mixture $g$-prior case,

$$
\log \left(\int P(\boldsymbol{Y} \mid \tilde{\boldsymbol{\theta}}, m) \pi(\tilde{\boldsymbol{\theta}}) d \tilde{\boldsymbol{\theta}}\right) \approx \frac{d}{2} \log (2 \pi)-\frac{1}{2} \log \left|-H^{\star}\right|+\log \left(P\left(\boldsymbol{Y} \mid \tilde{\boldsymbol{\theta}}^{\star}, m\right)\right)
$$


where $\tilde{\boldsymbol{\theta}}^{\star}$ is the mode of $P(\boldsymbol{Y} \mid \tilde{\boldsymbol{\theta}}, m) \pi(\tilde{\boldsymbol{\theta}})$ and $H^{\star}$ is the Hessian matrix evaluated at $\tilde{\boldsymbol{\theta}}^{\star}$. We compute these values with the functions optim and numDeriv: :hessian, respectively. We make a similar computation for $\int P(\boldsymbol{Y} \mid \tilde{\boldsymbol{\theta}}, m)^{b} \pi(\tilde{\boldsymbol{\theta}}) d \tilde{\boldsymbol{\theta}}$ to compute the fractional marginal model probability $q^{b}(\boldsymbol{Y} \mid \boldsymbol{\theta})$ for all $m \in \mathcal{M}$, well defined for both homoscedastic and heteroscedastic models. Once log-fractional marginal likelihoods have been computed for all models, we subtract the maximum from this set so that the set of log-fractional marginal likelihoods has been rescaled to have a maximum of 0 . Each value is exponentiated to obtain a set of fractional marginal likelihoods with maximum 1. This adjustment helps to avoid numerical underflow when computing posterior model probabilities.

\subsubsection{Model Priors}

With this adjusted set of fractional marginal likelihoods, we next consider the priors for the model space. The package slgf imposes a uniform prior by model class, and for classes containing multiple models, the prior on each class is uniformly divided among the models it contains. We finally compute posterior model probabilities for each model:

$$
P\left(m^{\prime} \mid \boldsymbol{Y}\right)=\frac{P\left(\boldsymbol{Y} \mid m^{\prime}\right) P\left(m^{\prime}\right)}{\sum_{\mathcal{M}} P(\boldsymbol{Y} \mid m) P(m)}
$$

\subsubsection{Parameter Estimation}

We compute estimators for the regression parameters, variance(s), and $g$ (when the ZellnerSiow mixture $g$-prior is used). Estimation of the regression parameters is straightforward:

$$
\hat{\boldsymbol{\beta}}=\underset{\boldsymbol{\beta}}{\arg \max } P(\boldsymbol{Y} \mid X, \boldsymbol{\beta}, \Sigma)
$$

so that $\hat{\boldsymbol{\beta}}=\left(X^{T} X\right)^{-1} X^{T} \boldsymbol{Y}$. Estimation of $\hat{\Sigma}$ and $g$ is more intensive. Recall we transform $\lambda=\log \sigma^{2}$ for one or both variances included in a model; let $\hat{\boldsymbol{\lambda}}:=\{\hat{\lambda}\}$ in homoscedastic 
models or $\left\{\hat{\lambda}_{1}, \hat{\lambda}_{2}\right\}$ in heteroscedastic models. Then

$$
\hat{\boldsymbol{\lambda}}=\underset{\boldsymbol{\lambda}}{\arg \max } \int P(\boldsymbol{Y} \mid X, \boldsymbol{\beta}, \Sigma) P(\boldsymbol{\beta}) P(\Sigma) d \boldsymbol{\beta}
$$

or,

$$
\{\hat{\boldsymbol{\lambda}}, \hat{g}\}=\underset{\boldsymbol{\lambda}, g}{\arg \max } \int P(\boldsymbol{Y} \mid X, \boldsymbol{\beta}, \Sigma, g) P(\alpha) P\left(\boldsymbol{\beta}_{-\alpha} \mid \Sigma\right) P(g) d \boldsymbol{\beta}
$$

Then, $\hat{\boldsymbol{\sigma}}^{2}=\exp \{\hat{\boldsymbol{\lambda}}\}$ for $\hat{\boldsymbol{\sigma}}^{2}=\left\{\hat{\sigma}^{2}\right\}$ or $\hat{\boldsymbol{\sigma}}^{2}=\left\{\hat{\sigma}_{1}^{2}, \hat{\sigma}_{2}^{2}\right\}$.

\subsection{Functionality}

The function lm.slgf requires several inputs to compute and output posterior model probabilities for all models and model classes of interest. The user begins with a dataframe containing a continuous response, at least one categorical predictor, and any other covariates of interest. To prevent confusion with the SLGF methodology, this dataframe cannot contain column names with the character string "group". The user must first identify a suspected latent grouping factor, usually by plotting the data and noting a latent structure within the levels of a categorical predictor as illustrated in Section 3.1. The user indicates, via the arguments response and lgf, character strings corresponding to the response and the suspected latent grouping factor variable names, respectively.

Next the user determines the model classes they wish to evaluate. We note the distinction between these model classes and the $\mathrm{R}$ class of a variable. The argument usermodels is a list where each element contains a string of R class formula or character. The user also specifies which classes should also be considered in a heteroscedastic context via the argument het, which provides an indicator 1 or 0 corresponding to each model class specified in usermodels. Together the arguments usermodels and het create the full set of model classes considered.

Next the user chooses a prior to place on the regression effects. As described in Section 3.2.2, prior="flat" (the default) implements the noninformative prior and prior="zs" 
imposes the Zellner-Siow mixture $g$-prior.

Finally the user must specify the minimum number of levels of the SLGF that can comprise a group, via the argument min. levels, which defaults to 1 . Because the number of possible grouping schemes increases exponentially with $K$, the user can reduce the number of candidate models, and hence speed up the computation, by increasing min. levels; however, because we partition into two groups, note min. levels may not exceed $\frac{K+1}{2}$. Additionally, when considering data with limited degrees of freedom, increasing min.levels can also ensure estimability of the specified usermodels; see Section 3.4.3 for more detail.

\subsection{Illustrations}

We next demonstrate the implementation of this methodology with the $\mathrm{R}$ package slgf.

\subsubsection{ANOVA}

First we revisit the dataset (denoted smell) analyzed by O'Brien and Heft [1995], who measured olfactory acuity olf on a continuous scale as a function of age agecat, where age groups were divided into five categorical levels. We note that levels 4 and 5 of the categorical predictor, age category, appear to have higher variance than levels 1, 2, and 3, but most standard analyses assume homoscedasticity. We consider the usual analysis comparing the null model, with a single mean, versus the alternative model, with 4 degrees of freedom for the mean effects, with homoscedastic error variance. Note we obtain maximum likelihood estimates for the single variance of $\hat{\sigma}_{\text {Null }}^{2}=0.04334$ and $\hat{\sigma}_{\text {Alt }}^{2}=0.03211$ for the null and alternative models, respectively. Instead consider agecat as the suspected latent grouping factor. The apparent latent grouping structure we observe is denoted 1,2,3:4,5 (or equivalently, 4,5:1,2,3). The means may also differ by level, but this is more difficult to distinguish by the plot. Thus we first consider the following model and heteroscedasticity 


\section{O'Brien and Heft (1995) Smell Data}

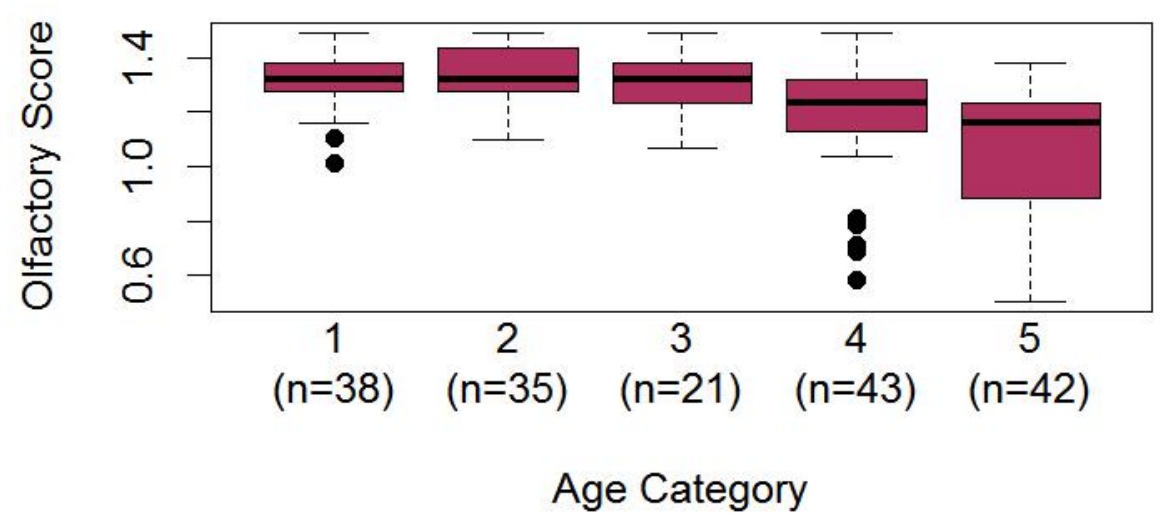

Figure 3.2: O'Brien and Heft [1995] studied olfactory function by age ( $y$-axis), where age was divided into five categories ( $x$-axis). The data suggests potential heteroscedasticity, with latent grouping structure 1,2,3:4,5.

structures: smell.models <- list("olf $\sim 1 "$, "olf $\sim$ agecat", "olf $\sim$ group") smell.het <- $c(0,0,1)$ This elicits four model classes: a homoscedastic global mean, homoscedastic age level means, homoscedastic group-based means, and group-based means with group-based heteroscedasticity. Finally we note that with a relatively large sample size, we prefer the use of noninformative priors via prior=" $f l a t "$, and we specify min. levels=1, as we have no prior information on the number of levels of agecat that may be grouped together and we wish to consider a comprehensive set of candidate models. The number of unique classes can always be obtained as length(usermodels)+sum(het).

smell. out <- lm.slgf(df=smell, response="olf", lgf="agecat", usermodels=smellmodels, prior="flat", het=c $(0,0,1)$, min.levels $=1)$

The output is a list of class slgf, with six elements when prior="flat" and seven when prior="zs":

- results, an $M \times 11$ matrix containing the model selection results and information 
for each model, including Model, the formula associated with each model; Scheme, the grouping scheme associated with each model; Variance, a string of either Homosk or Heterosk associated with each model; logFlik, the log-factional marginal likelihood associated with each model; Mod.Prior, the model prior associated with each model; Fmodprob, the posterior fractional model probability associated with each model; Cumulative, the cumulative posterior model probability of the models aggregated in descending order; $\mathrm{df}$. Index, an indicator of which element of group. df s contains the corresponding data.frame associated with each model's scheme; mle.index, mle.index, an indicator of which element of coefficients, variances, and gs contains the estimators associated with each model; Model. Index, an indicator of each model's rank by posterior model probability; and Class, denoting the model class associated with each model

- group.dfs, a list containing dataframes associated with each model class containing the appropriate effects, including group effects

- scheme.Probs, a $S \times 1$ data.frame containing the total probability for each grouping scheme considered

- class.Probs, a $C \times 1$ data.frame containing the total probability for each model class considered

- coefficients, MLEs for each model's regression effects

- variances, REML estimates for each model's variance(s)

- gs, REML estimates for each model's $g$; only included if prior="zs"

We summarize the five most probable models:

smell.out $\$$ results $[1: 5, c(1: 3,6,11)]$

Model Scheme Variance modprob.FBF Class 
1 olf group 4,5:1,2,3 Heterosk

2 olf agecat None Homosk

3 olf group 5:1,2,3,4 Heterosk

4 olf group 4:1,2,3,5 Heterosk

5 olf group 4,5:1,2,3 Homosk 1e+00 olf group, Heterosk

1e-08 olf agecat, Homosk

Oe+00 olf group, Heterosk

Oe+00 olf group, Heterosk

Oe+00 olf group, Homosk

Note we overwhelmingly favor the model with group-based mean effects and variances via scheme 4,5:1,2,3. Recall that our variance MLEs were $\hat{\sigma}_{\text {Null }}^{2}=0.04344$ and $\hat{\sigma}_{\text {Alt }}^{2}=0.03211$. However, with our method we obtain two REML estimators:

$>$ smell.hats <- extract.hats (smell. out, model.index=1)

$>\operatorname{print}(\mathrm{smell}$.hats\$`sigsq.4,5`)

[1] 0.05869897

$>\operatorname{print}($ smell.hats\$'sigsq.1,2,3`)

[1] 0.01211161

The function extract. hats provides the estimators for the coefficient(s) and variance(s) for a given model.index, as well as $g$ if prior="zs". That is, we compute REML estimates $\hat{\sigma}_{1,2,3}^{2}=0.05869897$ and $\hat{\sigma}_{4,5}^{2}=0.01211161$. This suggests that the standard methods overestimated the variance for three levels of the categorical predictor, and underestimated the variance for the other two.

Let us also consider the case where het $=c(1,1,1)$; that is, we include two additional classes: group-based variances and a single global mean, and group-based variances with means by agecat.

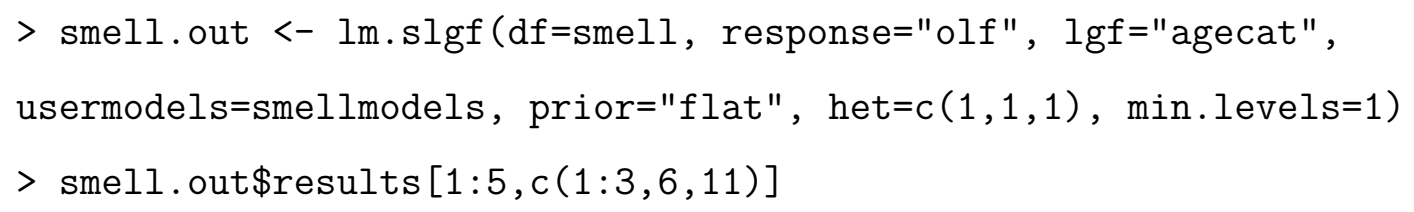


1 olf group 4,5:1,2,3 Heterosk 0.76422666 olf group, Heterosk

2 olf agecat 4,5:1,2,3 Heterosk 0.23577298 olf agecat, Heterosk

3 olf agecat 1,2:3,4,5 Heterosk 0.00000012 olf agecat, Heterosk

4 olf agecat 5:1,2,3,4 Heterosk 0.00000010 olf agecat, Heterosk

5 olf agecat 1:2,3,4,5 Heterosk 0.00000007 olf agecat, Heterosk

Now the most probable models are a bit less conclusive, as the distinct category-means model with scheme 4,5:1,2,3 group-based heteroscedasticity accounts for a meaningful amount of posterior model probability. We can easily summarize the scheme and class probabilities, which overwhelmingly favor scheme 4,5:1,2,3 and moderately favor the group-based means and variances model class:

$>$ smell.out\$scheme.Probs

Scheme.Prob

$4,5: 1,2,3 \quad 0.99999968$

$1,2: 3,4,5 \quad 0.00000012$

$5: 1,2,3,4 \quad 0.00000010$

$1: 2,3,4,5 \quad 0.00000007$

$2: 1,3,4,5 \quad 0.00000001$

None $\quad 0.00000001$

$1,3: 2,4,5 \quad 0.00000000$

$1,4: 2,3,5 \quad 0.00000000$

$1,5: 2,3,4 \quad 0.00000000$

$2,3: 1,4,5 \quad 0.00000000$

$2,4: 1,3,5 \quad 0.00000000$

$2,5: 1,3,4 \quad 0.00000000$

$3,4: 1,2,5 \quad 0.00000000$

$3,5: 1,2,4 \quad 0.00000000$

$3: 1,2,4,5 \quad 0.00000000$ 


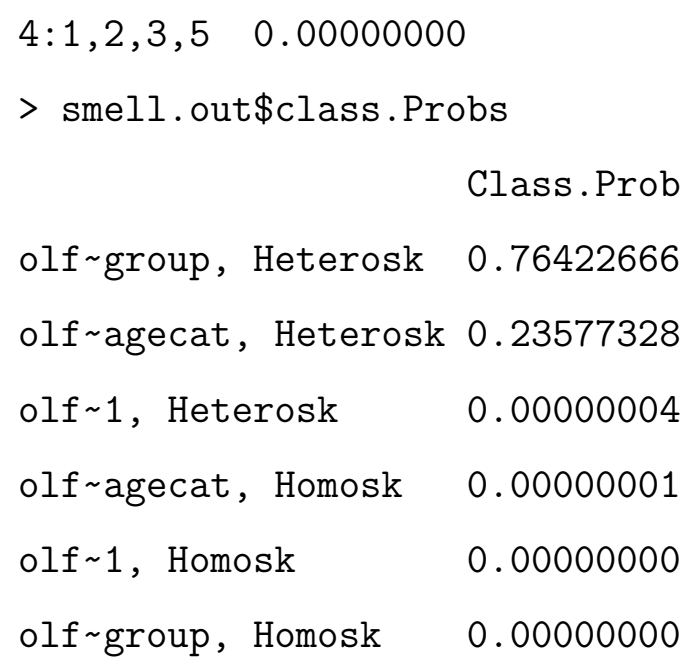

\subsubsection{Analysis of Covariance (ANCOVA)}

Next consider the data of Flurry [1939] in which the breaking strength of a starch chip (measured in grams) is analyzed according to the thickness of the chip (measured in $10^{-4}$ inches) and the type of starch used to create the chip (canna, corn, or potato starch). As usual, we begin by plotting the data to ascertain whether there is a latent grouping factor present. By inspection we note that the potato chips, represented by squares, appear to have a higher variability than the corn (triangles) and canna (squares) chips. A researcher's first approach might be analysis of covariance (ANCOVA), in which three parallel lines for each level of starch would be fit with a common error variance:

break.anc <- $\operatorname{lm}($ chips\$strength chips\$starch + chips\$film)

break.coefs <- break.anc\$coefficients

This leads to the following fit and residuals: We also note that a researcher's first inclination when faced with this classic cone-shaped residual pattern is to use a logarithmic transformation on the response. Let us implement this transformation and consider whether it has improved the apparent heteroscedasticity within the data:

logbreak.anc <- $\operatorname{lm}(\log ($ chips\$strength) $\sim \operatorname{chips\$ starch~+~chips\$ film)~}$ 
Flurry (1939) Data

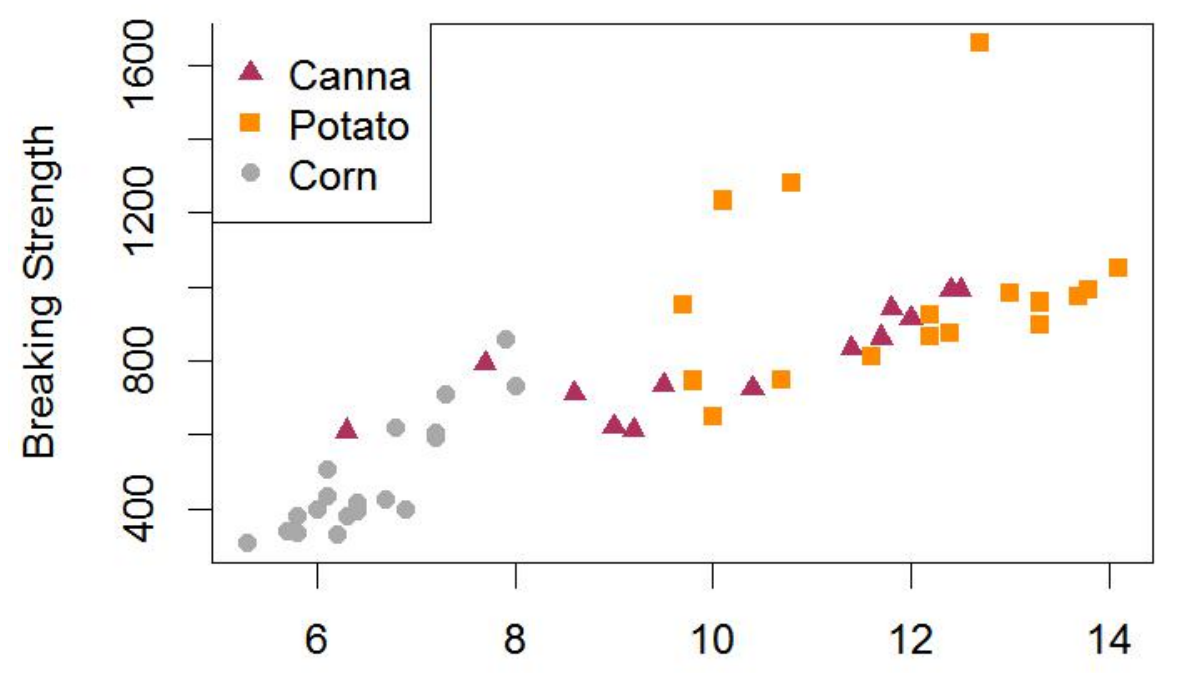

Film Thickness

Figure 3.3: The Flurry [1939] data suggests potential heteroscedasticity or a latent group interaction, with scheme potato:corn,canna. 


\section{Flurry (1939) Data}

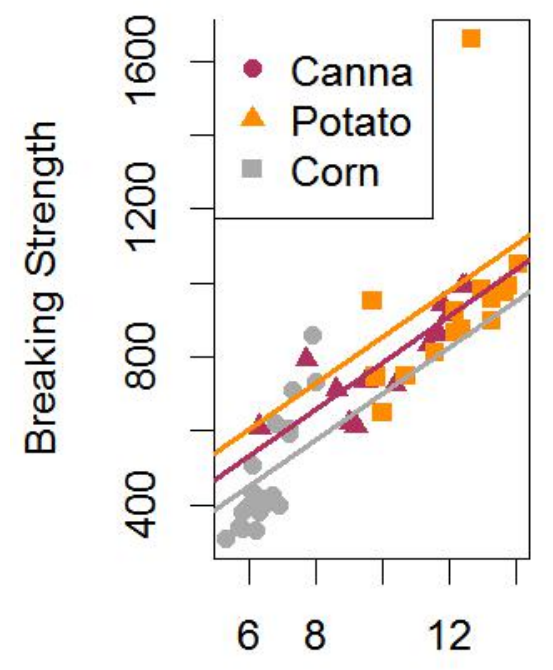

Film Thickness
Flurry (1939) ANCOVA Residuals

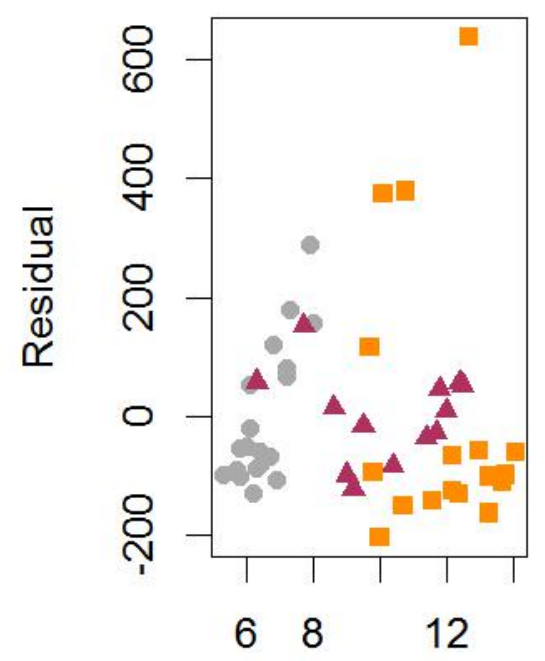

Index

Figure 3.4: An ANCOVA model fit to the Flurry [1939] data leads to a problematic residual pattern. 


\section{Flurry (1939) ANCOVA Residuals}

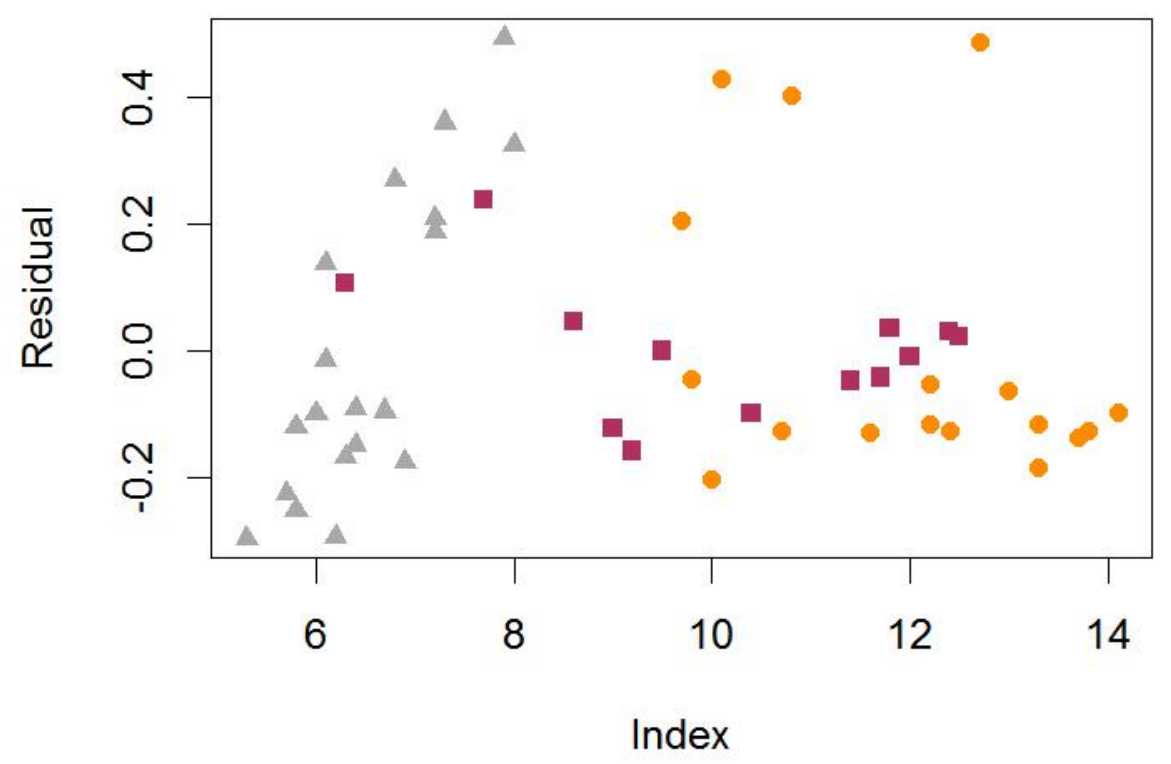

Figure 3.5: Residuals on the log-transformed Flurry [1939] data set show an alleviated cone shape, but the heteroscedasticity pattern remains by the levels of starch.

Although the conical shape appears to have been alleviated, we note that a more subtle pattern remains. As film thickness increases, the canna chips do not appear to show a similar increase in variance, so a log-transformation has failed to account for the variance structure that is a function of the categorical covariate, rather than the continuous film thickness. Thus we instead consider starch as a latent grouping factor. Additionally, we consider models with and without interactions between the categorical and continuous predictors:

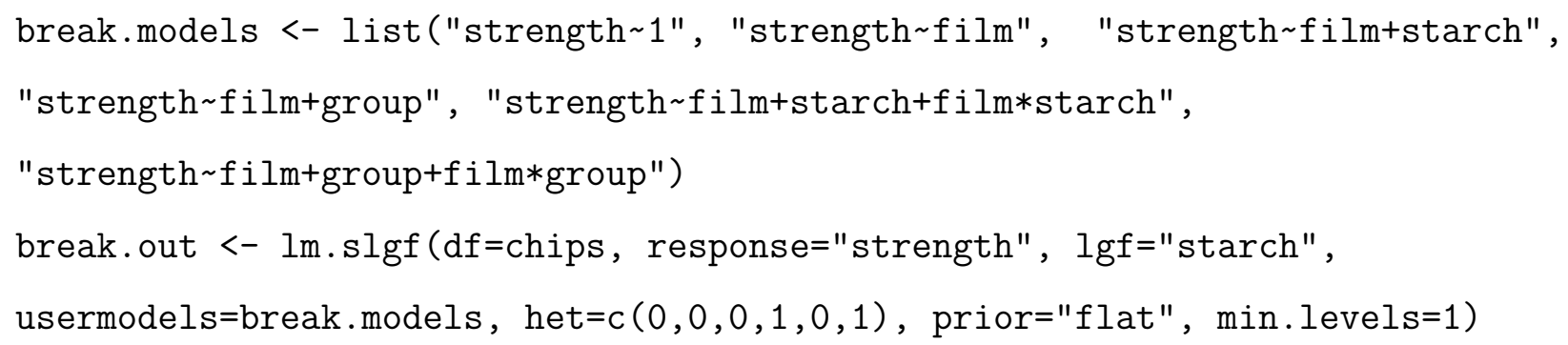


We note that the posterior model probabilities favor group-based heteroscedasticity with the scheme canna,corn:potato:

$>$ break.out\$results $[1: 5, c(1: 3,6)]$

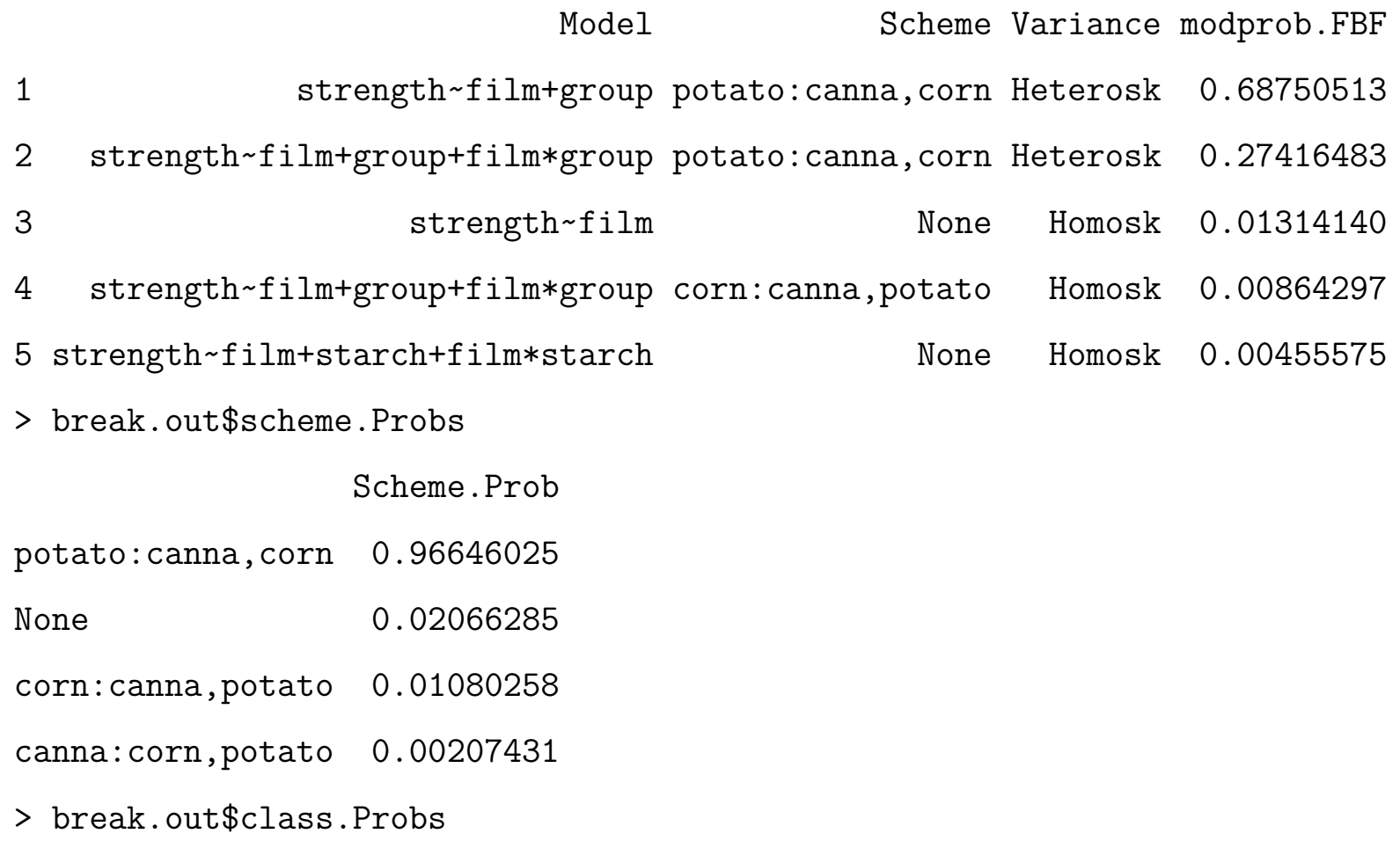

Thus we strongly conclude group-based heteroscedasticity with scheme potato:canna,corn along with main effects for film and group. The inclusion of a film*group interaction is 
less certain; we briefly revisit this uncertainty in Chapter ??.

\subsubsection{Two-way Replicated Layouts}

We revisit the two-way replicated layout of Meek and Ozgur [1991] illustrated in the right panel of Figure 3.1, where the torque required to tighten a locknut was measured as a function of a plating process and a threading method. As usual, we begin by plotting the data to assess for a latent grouping structure in Figure 3.6. Perhaps the first characteristic we note is

\section{Meek and Ozgur (1991) Locknut Data}

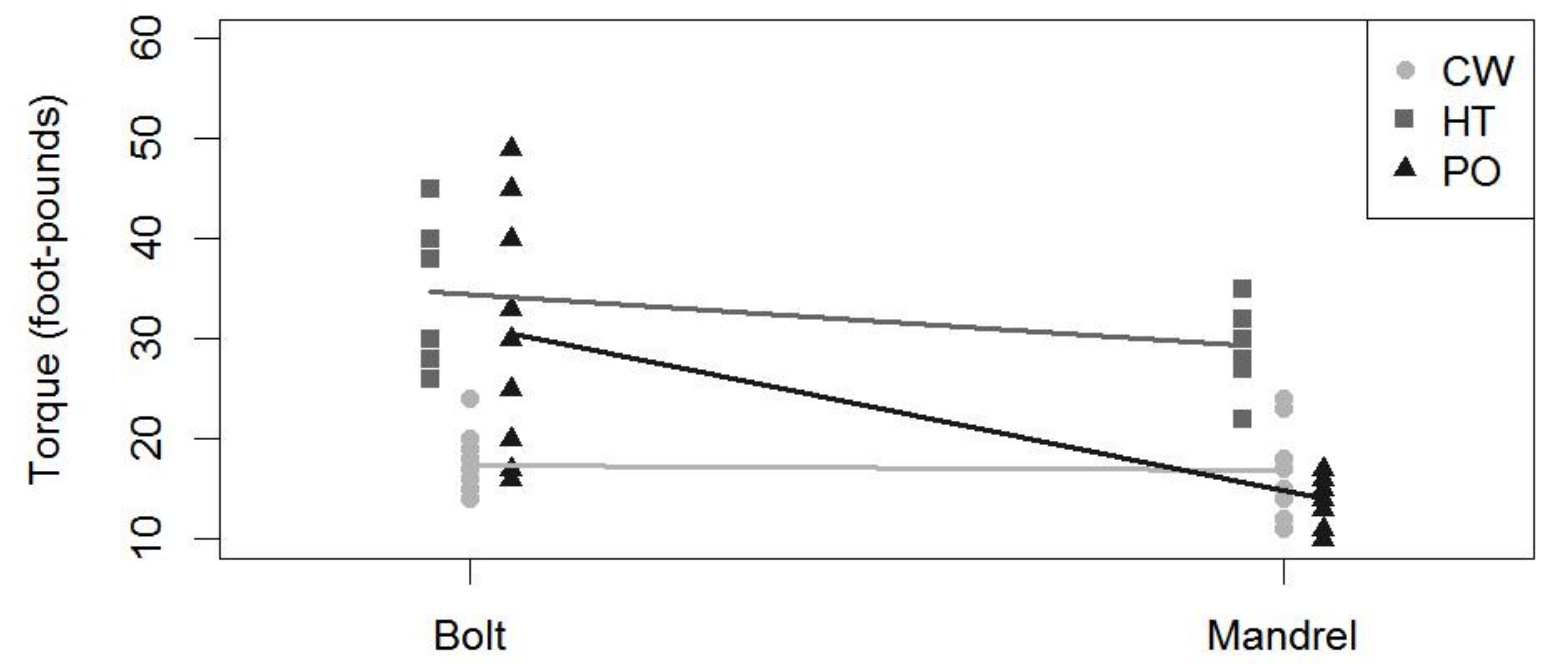

Threading Method

Figure 3.6: The torque ( $y$-axis) required to tighten a locknut is presented as a function of two predictors. First, the fixture on which the nut was threaded, either a bolt or a conical mandrel, and second, one of three plating methods including cadmium and wax, heat treating, and phosphate and oil, denoted CW, HT, and PO, respectively. Lines connect the means of the plating treatments at each level of fixture. The observations at level bolt appear to have a higher error variance than those of mandrel.

that the variance of the torque measured on locknuts fitted on bolts appear higher than those fitted on a mandrel. If we neglected to account for this potential heteroscedasticity, and fit 
only a standard homoscedastic interaction model, we would obtain the following coefficients and error variance estimate:

locknut.twoway <- $\operatorname{lm}($ Torque $\sim$ Fixture+Plating+Fixture $*$ Plating, data=locknut)

$>$ locknut.twoway\$coefficients

(Intercept)

17.4

PlatingHT

$-0.5$

Fixturemandrel : PlatingPO

$-15.9$

$>$ summary(locknut.twoway)\$sigma`2

\section{[1] 36.57778}

Instead we treat the fixture as the latent grouping factor. Note that with only two levels of the categorical predictor, there is only one trivial grouping scheme, bold:mandrel. Moreover, we can only consider distinct variances by group, as a group effect with one degree of freedom would be isomorphic to the standard fixture effect; thus it is important that we do not include the group term in the usermodels argument, but rather specify heteroscedastic models and lgf="Fixture". Regarding the regression effects, there appears to be an interaction between fixture and plating, as indicated by the relatively non-parallel lines. Thus we consider usermodels=list ("Torque 1 ", "Torque Fixture", "Torque $\sim$ Fixture+Plating", "Torque $\sim$ Fixture*Plating") and het=c $(1,1,1,1)$. Because there are 10 replicates at each combination of factor levels of fixture and plating, we use the noninformative prior prior="flat".

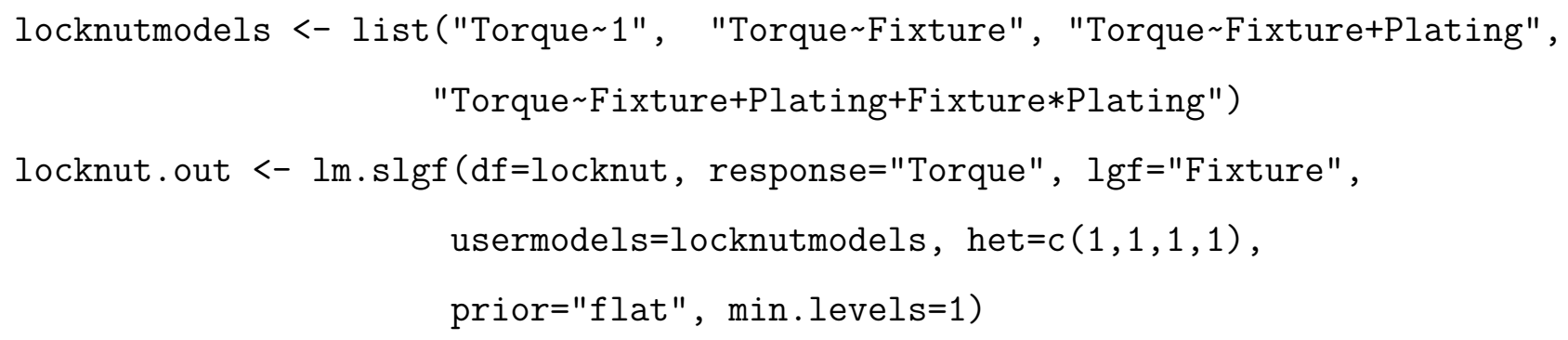


With relatively few models, and only one trivial grouping scheme in heteroscedastic classes, we view all eight candidate models' posterior probability. Because each model class contains only a single model, each model probability is also its corresponding class probability:

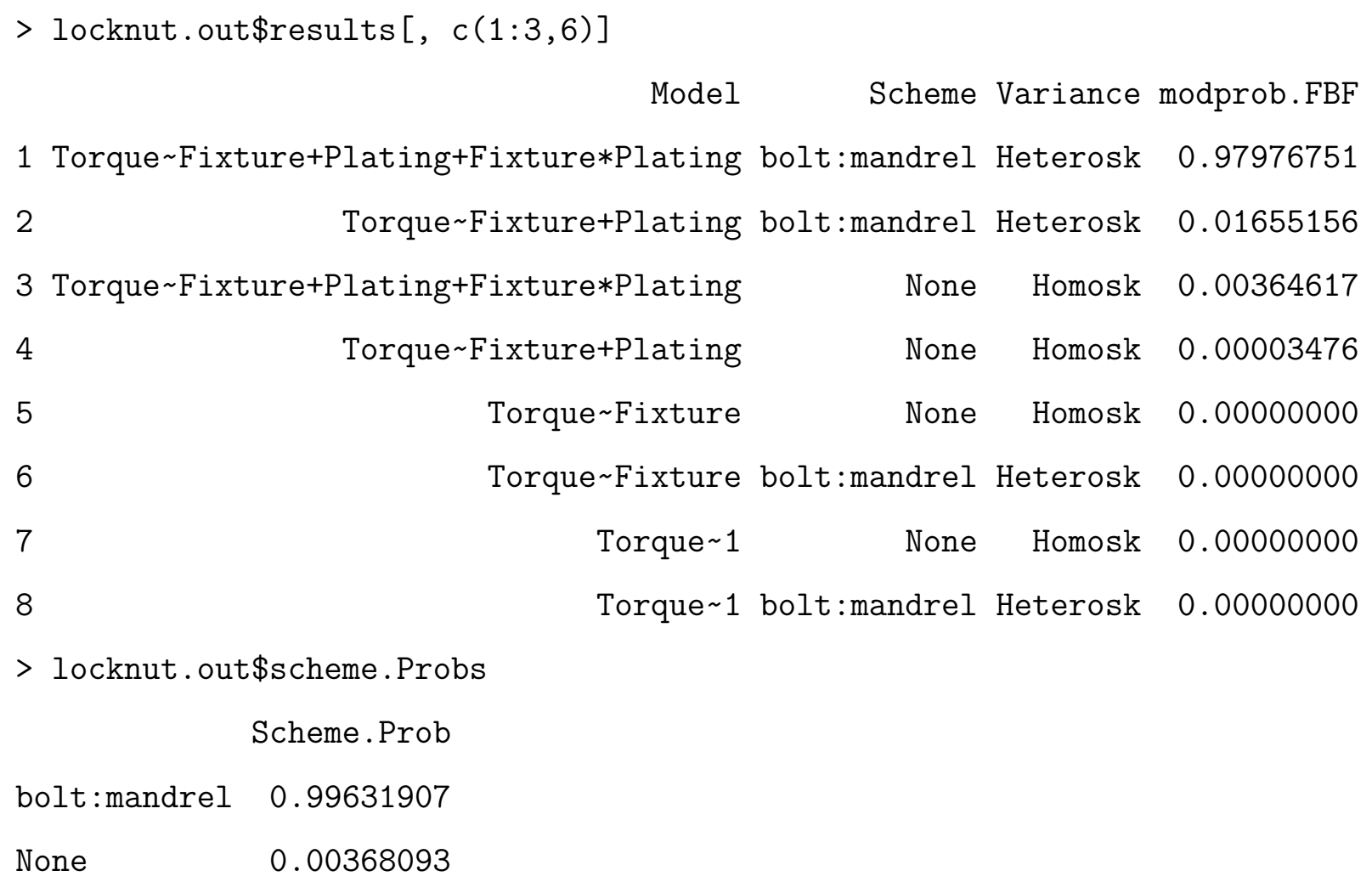

The most probable model accounts for approximately $98 \%$ of the posterior mass, indicating distinct variances between bolt and mandrel, along with a fixture by plating interaction. Our estimates for the coefficients are equivalent to the least squares estimates obtained from lm:

locknut.out\$coefficients [ [9] ]

$\begin{array}{rrr}\text { (Intercept) } & \text { Fixturemandrel } & \text { PlatingHT } \\ 17.4 & -0.5 & 17.3\end{array}$

PlatingPO Fixturemandrel:PlatingHT Fixturemandrel:PlatingPO

$\begin{array}{lll}13.1 & -4.8 & -15.9\end{array}$ 
On the other hand, let us inspect this data more carefully. The most probable model, in the set locknut. models as parametrized earlier, is Torque Fixture+Plating+Fixture*Plating with heteroscedasticity between bolt and mandrel. We observe the residuals of this model; for the sake of plotting the data efficiently, we append a factor onto the locknut data that denotes the interaction using either " $b$ " or "m" for bolt and mandrel:

\section{Residuals of Full Interaction Model Torque $\sim$ Fixture + Plating + Fixture*Plating}

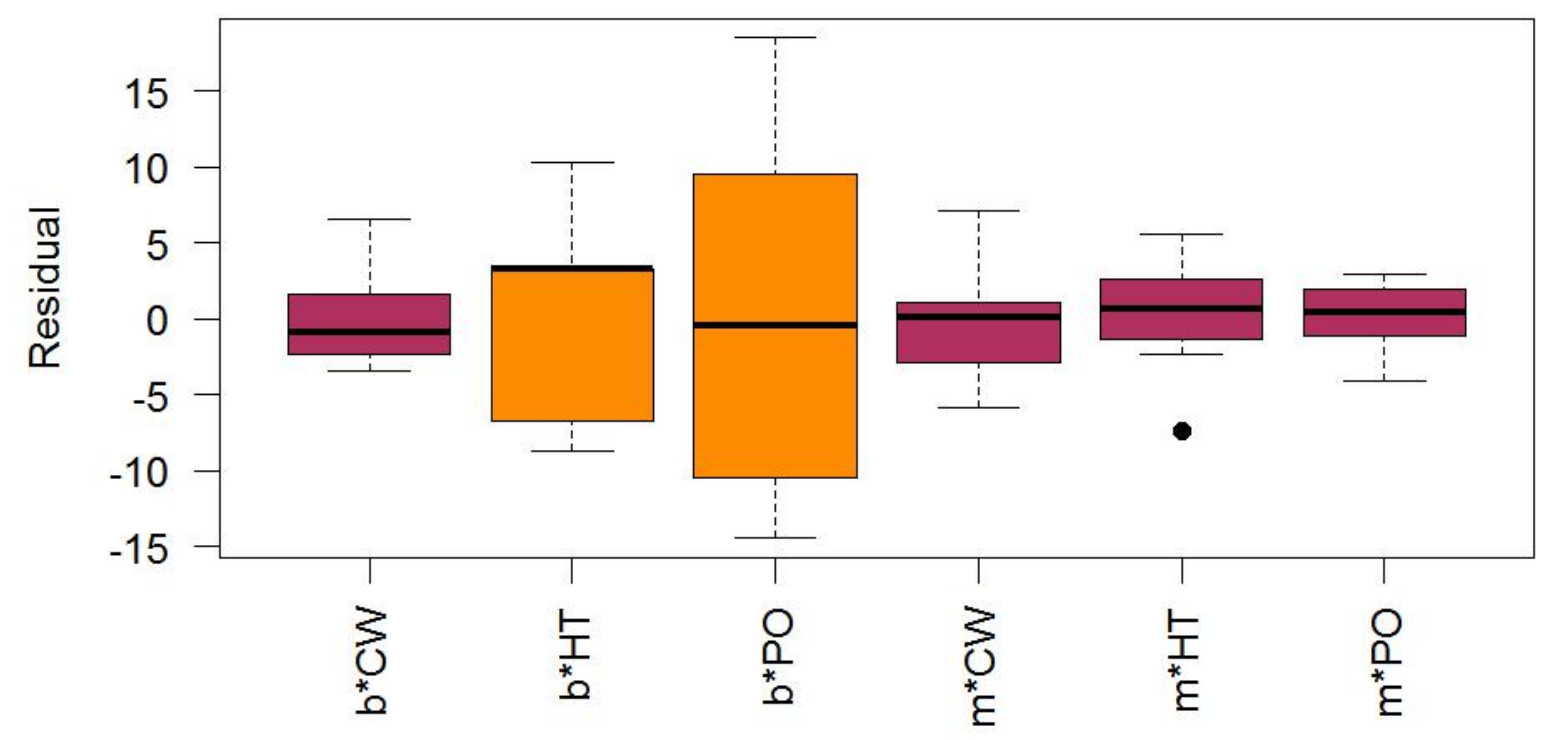

Figure 3.7: The residuals ( $y$-axis) under the full interaction model Torque Fixture+Plating + Fixture*Plating indicate there may be a more subtle heteroscedasticity structure than bolt:mandrel; we color-code for emphasis. We consider Fixture* Plating as the lgf instead.

locknut\$`Fixture*Plating`<- paste0 (substr(as.character(locknut\$Fixture), 1, 1), "*", locknut\$Plating)

It appears the latent grouping factor may in fact be the levels of the interaction itself, with equivalent means and distinct variances by scheme $\mathrm{b}^{*} \mathrm{CW}, \mathrm{m} * \mathrm{CW}, \mathrm{m} * \mathrm{HT}, \mathrm{m} * \mathrm{PO}: \mathrm{b}^{*} \mathrm{HT}, \mathrm{b}{ }^{*} \mathrm{PO}$. So alternatively we let usermodels contain only the full interaction model, and let 


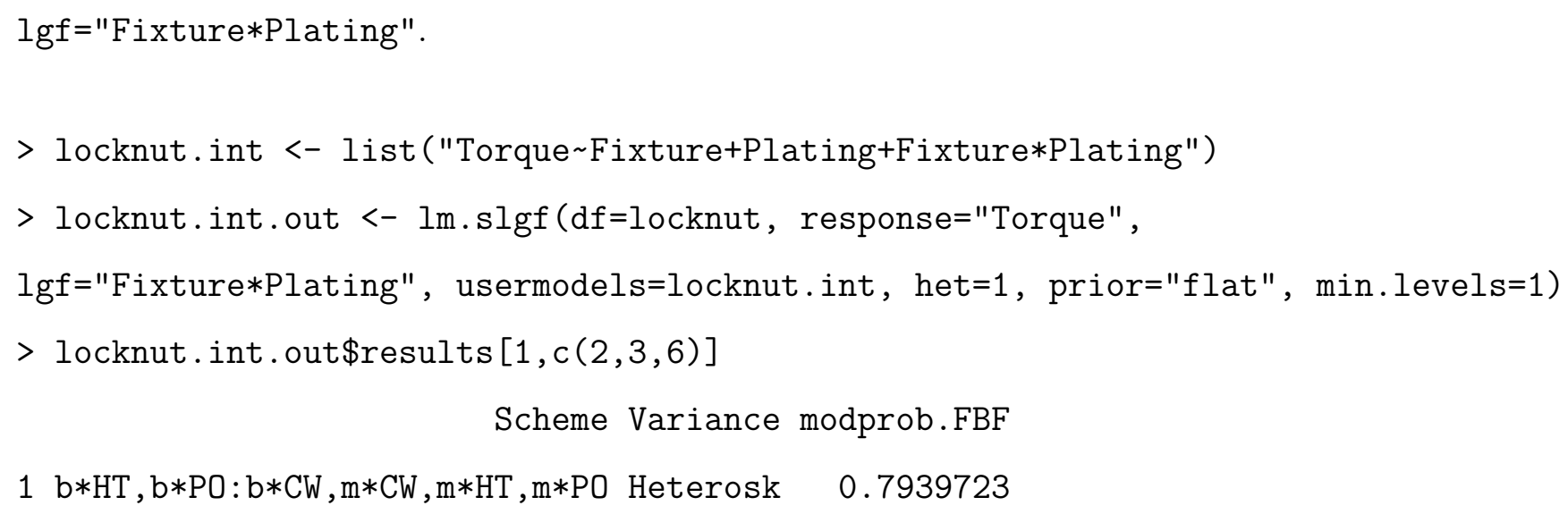

As expected from the residual boxplot, we place nearly $80 \%$ of the posterior mass on the model we suspected from the visual inspection. This illustrates the flexibility of the SLGF method, even allowing for latent groupings by the levels of an interaction effect. Additionally, our method can be implemented as a post-hoc analysis on the residuals of the levels of a categorical predictor based on an existing model.

\subsubsection{Two-way Unreplicated Layouts}

Next we restrict our two-way approach to an unreplicated context, which was the inspiration for the SLGF method. Consider the data analyzed by Franck [2018], where six dogs with lymphoma were studied. Two individual samples were taken from healthy and tumor tissue within each dog, and the copy number variation was measured for each sample. Without loss of generality, we arrange dogs into rows and tissue types into columns of a two-way layout. We first plot the data to determine whether a latent grouping structure underlies the data: We strongly suspect that dogs 1,2 , and 5 behave distinctly from $\operatorname{dogs} 3,4$, and 6 . The telltale non-parallel lines suggest an underlying interaction, but with only a single observation in each cell, we lack the degrees of freedom to fit a standard row by column interaction term. Instead, we let dog represent the SLGF, exclude the column effect, and specify a group-bycolumn interaction term, isomorphic to fitting distinct column effects by group. Thus we consider four reasonable model classes: a dog and tissue effect, a dog and tissue-by-column 
Franck et. al. (2013)

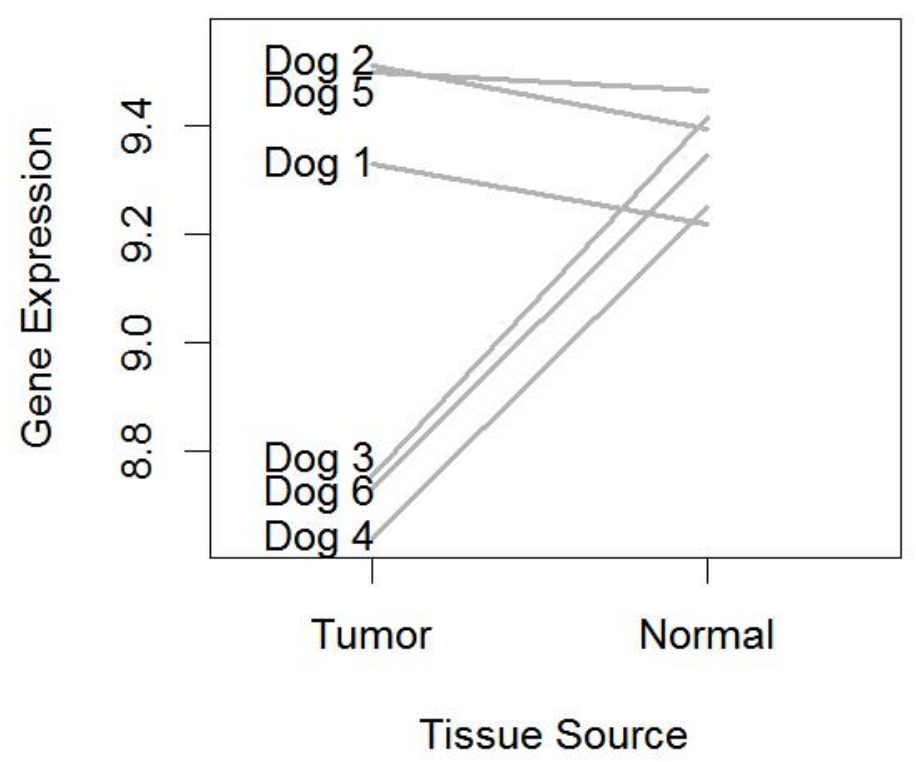

Figure 3.8: The data analyzed by Franck et al. [2013] measured a continuous gene expression response (y-axis) in six dogs with lymphoma. Tissue samples were taken from tumor and normal tissue in each dog. 
interaction, and the heteroscedastic counterparts of each class. We accomplish this with the arguments usermodels=list ("gene dog+tissue", "gene dog+group:tissue") and het $=c(1,1)$. Additionally, we must specify min .1 evels=2 or min .1 evels $=3$ to ensure sufficient degrees of freedom to estimate the group: col effect. Here we consider min . levels=2 in the interest of assessing a more complete set of candidate models.

Because of the limited amount of data, our choice of prior is more impactful in this case. We impose prior="zs" to utilize the Zellner-Siow mixture $g$-prior, as the fractional Bayes factor exponent would require a prohibitively high proportion of the data for model training.

We first put the two-way layout into a data.frame format compatible with the slgf function, and then implement this approach:

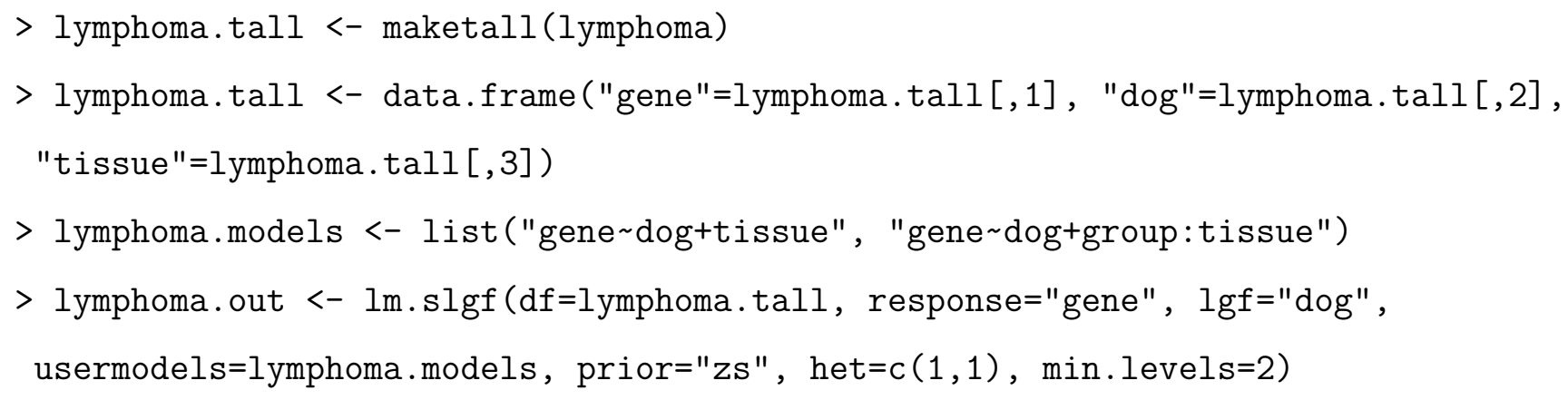

Note the : operator in the usermodels syntax, which does not automatically include the main effects group and tissue which are not both estimable. As expected, we conclude with high probability that scheme 1,2,5:3,4,6 underlies the data. The five most probable models are given by:

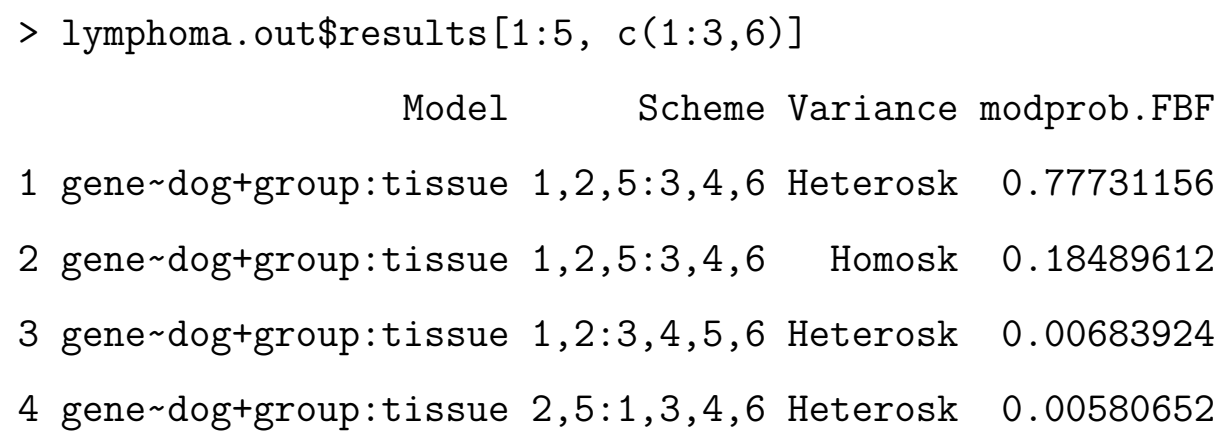




$$
\text { gene } \sim \text { dogttissue None Homosk } 0.00429596
$$

while the class and five highest scheme probabilities are

$$
\begin{aligned}
& \text { > lymphoma.out\$class.Probs } \\
& \text { Class.Prob } \\
& \text { gene dog+group:tissue, Heterosk } 0.79638854 \\
& \text { gene dog+group:tissue, Homosk } 0.19069556 \\
& \text { gene dog+tissue, Heterosk } 0.00861996 \\
& \text { gene dog+tissue, Homosk } 0.00429596 \\
& >\text { head (lymphoma.out\$scheme.Probs, 5) } \\
& \text { Scheme.Prob } \\
& 1,2,5: 3,4,6 \quad 0.96631751 \\
& 1,2: 3,4,5,6 \quad 0.00790504 \\
& 2,5: 1,3,4,6 \quad 0.00723547 \\
& \text { None } \quad 0.00429596 \\
& 1,5: 2,3,4,6 \quad 0.00365555
\end{aligned}
$$

The group.dfs element of lymphoma.out contains data.frames associated with each model and grouping scheme. We first determine which element of 1ymphoma.out\$group.dfs contains the data.frame of interest via the column df. Index:

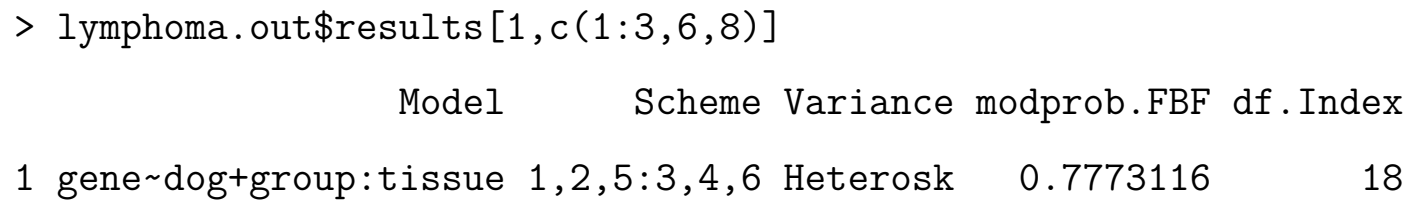

This tells us that element 18 of lymphoma.out\$group.dfs contains the data.frame with the 1,2,5:3,4,6 group effect:

$>$ lymphoma.out\$group.dfs [[18]] 


\begin{tabular}{lrrrr}
\multicolumn{5}{c}{ gene dog tissue group } \\
1 & 9.3278 & 1 & 1 & $1,2,5$ \\
2 & 9.2168 & 1 & 2 & $1,2,5$ \\
3 & 9.5108 & 2 & 1 & $1,2,5$ \\
4 & 9.3942 & 2 & 2 & $1,2,5$ \\
5 & 8.7535 & 3 & 1 & $3,4,6$ \\
6 & 9.4158 & 3 & 2 & $3,4,6$ \\
7 & 8.6372 & 4 & $13,4,6$ \\
8 & 9.2480 & 4 & 2 & $3,4,6$ \\
9 & 9.4981 & 5 & 1 & $1,2,5$ \\
10 & 9.4626 & 5 & 2 & $1,2,5$ \\
11 & 8.7322 & 6 & 1 & $3,4,6$ \\
12 & 9.3439 & 6 & 2 & $3,4,6$
\end{tabular}

\subsubsection{Balanced Incomplete Block Design (BIBD)}

Finally we illustrate the utility of the Zellner-Siow mixture $g$-prior in another context with limited data, the balanced incomplete block design (BIBD). Recall the data described in the center panel of Figure 3.1 by Davies [1954], which measured the wear on tires as a function of the compound from which they were manufactured. Four tires were used in the study and treated as blocks. Four compounds were studied, but due to manufacturing limitations, each tire was divided into thirds and the sections made from three different compounds. Let us first visualize the data: We note that the abrasion appears higher for compounds $\mathrm{C}$ and $\mathrm{D}$, and for tire 4. Compounds $\mathrm{C}$ and $\mathrm{D}$ behave quite similarly over tires as well, as indicated by their similar V-shapes. A standard frequentist analysis considers a linear model with factors compound and as.factor(tire):

\footnotetext{
$>$ roadwear. $\operatorname{lm}<-\operatorname{lm}$ (abrasion compound + as.factor (tire), data=roadwear) $>$ anova(roadwear.lm)
} 


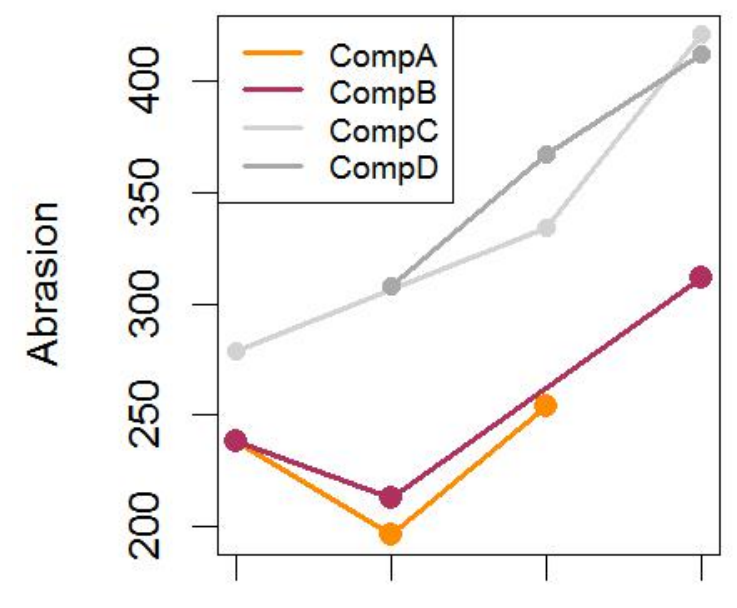

Tire 1 Tire 2 Tire 3 Tire 4

Tire

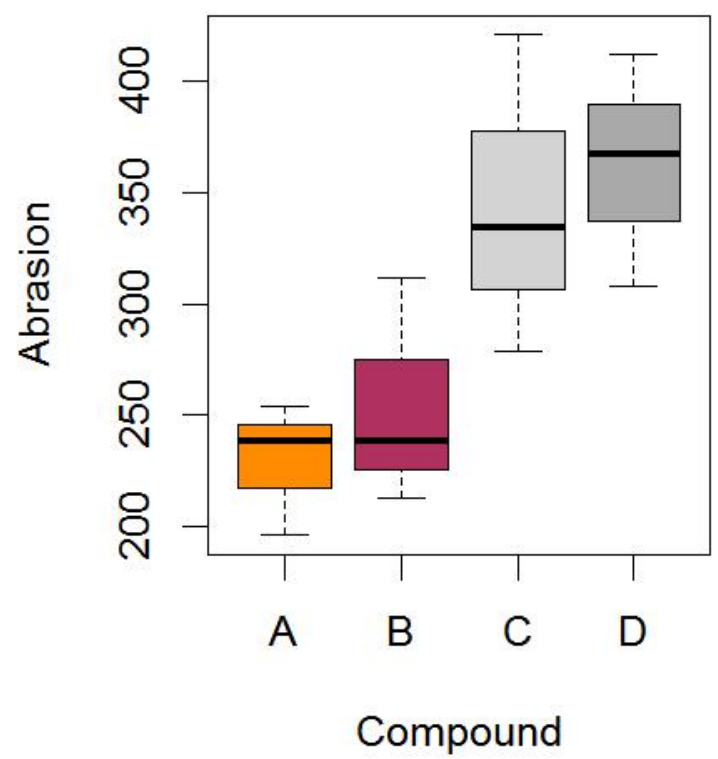

Figure 3.9: The data analyzed by Davies [1954] measured the abrasion on four tires (blocks), each manufactured from three of four compounds, in a road wear test. 
Analysis of Variance Table

Response: abrasion

Df Sum Sq Mean Sq F value $\operatorname{Pr}(>F)$

compound $\quad 3 \quad 3881412938.0 \quad 36.946 \quad 0.0007763 * * *$

as.factor(tire) $\quad 3 \quad 21038 \quad 7012.6 \quad 20.026 \quad 0.0032406 * *$

$\begin{array}{llll}\text { Residuals } & 5 & 1751 & 350.2\end{array}$

The analysis of variance indicates that the blocking factor tire was and important inclusion to account for variability in the data. Additionally, compound is a significant source of variation in the model. A reasonable follow-up is to conduct multiple comparisons with a post-hoc pairwise comparisons method; here we illustrate the Tukey's honest significant difference method, which indicates that compounds $\mathrm{A}$ and $\mathrm{B}$ are statistically equivalent, C and D are statistically equivalent, but all other pairwise comparisons (A vs. C, A vs. D, B vs. C, and B vs. D) show a significant difference.

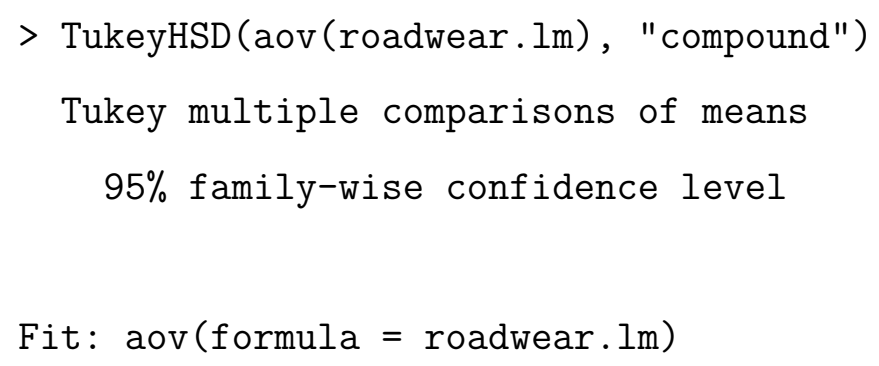




\section{D-C $\quad 17.66667 \quad-38.71244 \quad 74.04578 \quad 0.6755282$}

Alternatively, we consider our proposed method with compound treated as the SLGF:

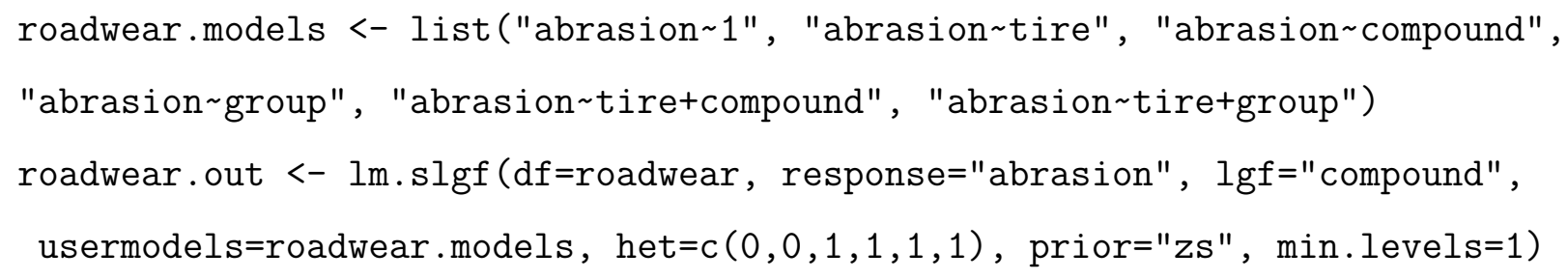

Many models receive a non-negligible proportion of the posterior model probability. The ten most probable models are given by:

$>$ roadwear.out\$results $[1: 10, c(1: 3,6)]$

\section{Model Scheme Variance modprob.FBF}

1 abrasion tire+group A,B:C,D Heterosk 0.24842160

2 abrasion tire+compound None Homosk 0.21495758

3 abrasion tire+group A,B:C,D Homosk 0.12682158

4 abrasion tire+compound D:A,B,C Heterosk 0.05072768

5 abrasion tire+compound A,B:C,D Heterosk 0.04032375

6 abrasion tire+compound A:B,C,D Heterosk 0.04031812

7 abrasion tire+compound A,C:B,D Heterosk 0.03974173

8 abrasion tire+compound C:A,B,D Heterosk 0.03718444

9 abrasion tire+compound A,D:B,C Heterosk 0.03625826

10 abrasion tire+compound B:A,C,D Heterosk 0.03478117

Note the most probable grouping structure, A,B:C,D, is the same one implied by the results of the TukeyHSD post-hoc comparison. However, note that nearly $60 \%$ of the posterior class probability belongs to heteroscedastic classes:

$>$ roadwear.hetprob <- sum(roadwear.out\$class.Probs [which (grepl ("Heterosk", 
rownames (roadwear. out $\$$ class. Probs))), ])

$>$ print (roadwear.hetprob)

[1] 0.5989437

In this context, our fully Bayesian analysis mostly agrees with the standard frequentist approach, but the package slgf easily allows the user to explore a red flag regarding potential latent heteroscedasticity.

\subsection{Summary}

We provide a user-friendly and intuitive $\mathrm{R}$ package, slgf, for researchers to implement the SLGF methodology in practice. The SLGF approach provides an intuitive, easy to implement analysis that has shown to be reasonable and beneficial in many common linear model examples. By partitioning the levels of a categorical predictor into two groups, we can detect latent structures including group-based regression effects, interactions, heteroscedasticity, and combinations of these. By selecting probable model classes and partitioning schemes with common priors, fractional Bayes factors, and Bayesian models selection, we provide a straightforward Bayesian approach. The package uses syntax similar to the lm function and allows for users to specify a broad range of model parametrizations that are highly customizable for varying needs, scientific contexts, and prior knowledge. 


\section{Chapter 4}

\section{Conclusions}

\subsection{Summary}

This work has aimed to provide an intuitive, flexible, mathematically and computationally feasible alternative to classical linear model analysis methods. Through the use of a combinatoric grouping approach, fractional Bayes factors, and Bayesian model selection, we can detect a wide variety of latent grouping structures underlying a dataset.

We first noted that there is a lack of literature addressing the specific concerns of latent group-based effects describe here. A foundation was then laid supporting our approach, including derivation of the posterior model probabilities, a fractional Bayes factor approach that accounts for our noninformative comparison between homoscedastic and heteroscedastic models, and a computational strategy for efficient and accurate calculations. We have demonstrated the applicability of our method through numerous real datasets that manifest the group-based effects we have described, illustrating a great utility for this work in a variety of applications. A simulation study was also undertaken to show our method's ability to detect a range of group-based effects in linear models of diverse types. Finally, an intuitive $R$ package, slgf, was written and thoroughly described so that researchers from many fields, 
particularly those outside of statistics, might be able to implement our methodology on their data.

\subsection{Future Work}

Future avenues of research are numerous and promising. We revisit the data of Flurry [1939] in Figure 4.1 to motivate our first future goal. Recall that we placed approximately $69 \%$ posterior model probability on the model with the film and a group-based effect, and group-based variances, with scheme canna,corn:potato. The next most probable model had the same heteroscedasticity scheme, with a film by group interaction as well. Thus we are quite confident in the group-based heteroscedasticity, but the regression effects are less certain. As we inspect Figure 4.1, we suspect that an issue may be that corn actually has a distinct regression effect from canna and potato. We thus propose a more flexible SLGF approach whereby there are two distinct suspected latent grouping factors: a regression effect SLGF and a variance SLGF. This could allow us not only to model the regression effects and variances with completely different categorical effects, but also do so with the same categorical effect but distinct schemes. We are optimistic that the existing methodology and $\mathrm{R}$ code is sufficient to make this extension with relative simplicity.

Next, we hope to explore statistical inference on parameters under the SLGF framework; promising preliminary results have been described by Franck [2018]. In many cases, the most difficult component of model averaging is computing model weights; that is, posterior model probabilities for the model in question. We believe the method described by Hoeting et al. [1999] has promise in our context.

A third future goal is to continually improve the $R$ package. The $R$ package slgf was developed over several months by aggregating three separate functions that had originally been written to accommodate only one-way ANOVA, ANCOVA, and two-way unreplicated data, respectively. These functions were then generalized so that they could accommodate 
Flurry (1939) Data

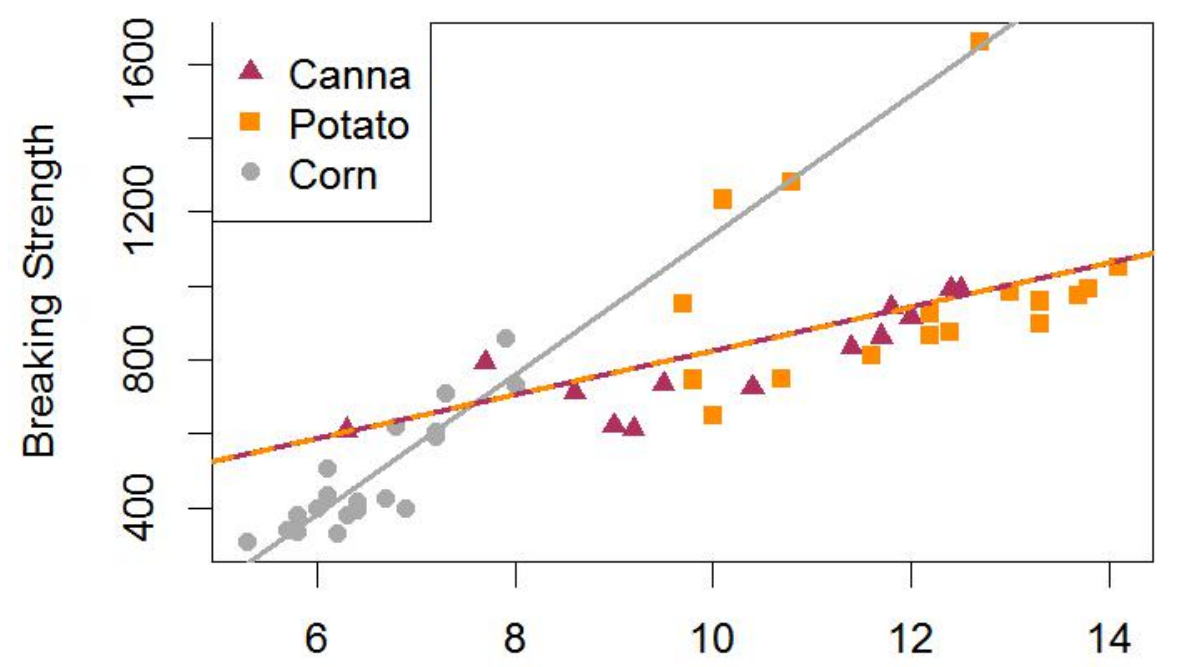

Film Thickness

Figure 4.1: On closer examination of the Flurry data [Flurry, 1939], it appears that the latent heteroscedasticity by scheme canna,corn:potato can be improved upon if we also model regression effects via the scheme corn:canna,potato. 
a broader class of linear models with a categorical predictor, as we have carefully developed our method so that it is very general and flexible. In its current form, although user-friendly and functional, there are many places to make the code and computations more efficient. For example, a first sequence computes the design matrices and regression effect MLEs for each model. Next a second sequence performs two tasks: it first estimates the variance and, if necessary, $g$, for each model, and then computes the posterior model probability for each model. This computation requires a relatively costly optimization over two or three dimensions, possibly for many model classes and grouping schemes. These sequences could be combined into one for loop, and, more efficient methods of optimization could be employed.

Generalization to three latent groups is another reasonable extension. Mathematically, this would be straightforward and could allow for more complex grouping structures to be accounted for. We are currently in search of datasets that reasonably illustrate such a latent grouping structure.

Finally, Gaussian process (GP) modeling is a burgeoning area of statistics. Latent categorical (qualitative) factors were recently discussed by Zhang et al. [2019], where they are modeled similarly to numeric variables with the goal of dimension reduction and eliciting parsimony. With our approach, we might consider distinct GPs according to the levels of a categorical predictor, perhaps with distinct group-based lengthscales, covariance functions, or nuggets.

It is our belief that the SLGF methodology outlined in this work can become a staple of many researchers' statistical analysis repertoires. Because of its broad scope, clear application in real datasets, and straightforward implementation in practice, we are optimistic that this work can assist researchers in many fields. 


\section{Bibliography}

M. S. Bartlett. Properties of sufficiency and statistical tests. Proceedings of the Royal Statistical Society, Series A, 160(901):268-282, May 1937.

James O. Berger and Luis R. Pericchi. The intrinsic bayes factor for model selection and prediction. Journal of the American Statistical Association, 91(433):109-122, 031996.

W. D. Berry. Quantitative Applications in the Social Sciences: Understanding Regression Assumptions. SAGE Publications Ltd., Thousand Oaks, CA, 1993.

P. J. Bickel. Using residuals robustly i: Tests for heteroscedasticity, nonlinearity. Annals of Statistics, 6:266-291, 1978.

W. John Boscardin and Andrew Gelman. Bayesian computation for parametric models of heteroscedasticity in the linear model, 1994.

G. E. Box and D. R. Cox. An analysis of transformations. Journal of the Royal Statistical Society, 26:211-252, 1964.

G. E. P. Box and W. J. Hill. Correcting inhomogeneity of variance with power transformation weighting. Technometrics, 16:385-389, 1974.

T. S. Breusch and A. R. Pagan. A simple test for heteroscedasticity and random coefficient variation. Econometrica (pre-1986), 47(5):1287, 09 1979. URL http://login.ezproxy.lib.vt.edu/login?url=https ://search-proquest-com . 
ezproxy.lib.vt.edu/docview/214655264?accountid=14826. Copyright - Copyright Econometric Society Sep 1979; Last updated - 2011-10-07; CODEN - ECMTA7.

Morton B. Brown and Alan B. Forsythe. Robust tests for the equality of variances. Journal of the American Statistical Association, Vol 69(346):364-367, June 1974.

Raymond J. Carroll and David Ruppert. Robust estimation in heteroscedastic linear models. The Annals of Statistics, 10(2):429-441, June 1982.

Raymond J. Carroll and David Ruppert. Transformation and weighting in regression. Chapman and Hall, New York, 1988.

R. Dennis Cook and Sanford Weisberg. Diagnostics for heteroscedasticity in regression. Biometrika, 70(1):1-10, April 1983. ISSN 0304-4076. doi: https://doi.org/10.1016/ 0304-4076(85)90158-7. URL http://www.sciencedirect.com/science/article/pii/ 0304407685901587.

J. G. Cragg. More efficient estimation in the presence of heteroscedasticity of unknown form. Econometrica, 51(3):751-763, May 1983.

F. Cribari-Neto and S. G. Zarkos. Bootstrap methods for heteroskedastic regression models: evidence on estimation and testing. Econometric Reviews, 18(2):211-228, 1999 . doi: 10.1080/07474939908800440. URL https://doi.org/10.1080/07474939908800440.

Edilberto Cepeda Cuervo and Jorge Alberto Achcar. Regression models with heteroscedasticity using bayesian approach. Revista Colombiana de Estadística, 32(2):267-287, December 2009.

O. L. Davies. The Design and Analysis of Industrial Experiments. Oliver \& Boyd, London, 1954.

John Deegan. The consequences of model misspecification in regression analysis. Multivariate Behavioral Research, 11(2):237 - 248, 1976. ISSN 0378-3758. doi: 10.1207/ s15327906mbr1102_9. URL https://doi.org/10.1207/s15327906mbr1102_9. 
Philippe J. Deschamps. On the estimated variances of regression coefficients in misspecified error components models. Econometric Theory, 7(3):369-384, September 1991.

Bianca Dumitrascu, Gregory Darnell, Julien Ayroles, and Barbara E. Engelhardt. A bayesian test to identify variance effects. Cornell University Library, 2015.

Friedhelm Eicker. Limit theorems for regressions with unequal and dependent errors. In Proceedings of the Fifth Berkeley Symposium on Mathematical Statistics and Probability, Volume 1: Statistics, pages 59-82, Berkeley, Calif., 1967. University of California Press. URL https://projecteuclid.org/euclid.bsmsp/1200512981.

M. S. Flurry. Breaking strength, elongation and folding endurance of films of starches and gelatin used in sizing. Technical Bulletin (United States Department of Agriculture), 674: $1-36,1939$.

Christopher Franck. Detection of hidden additivity and inference under model uncertainty for unreplicated factorial studies via bayesian model selection and averaging. Technometrics, 0(ja):1-30, 2018. doi: 10.1080/00401706.2018.1518791. URL https ://doi .org/10.1080/ 00401706.2018 .1518791 .

Christopher T. Franck and Jason A. Osborne. Exploring Interaction Effects in Two-Factor Studies using the hiddenf Package in R. The R Journal, 8(1):159-172, 2016. URL https: //journal.r-project.org/archive/2016/RJ-2016-011/index.html.

Christopher T. Franck, Dahlia M. Nielsen, and Jason A. Osborne. A method for detecting hidden additivity in two-factor unreplicated experiments. Computational Statistics $\&$ Data Analysis, 67(Supplement C):95 - 104, 2013. ISSN 0167-9473. doi: https://doi.org/ 10.1016/j.csda.2013.05.002. URL http://www.sciencedirect.com/science/article/ pii/S0167947313001618.

Paul Gilbert and Ravi Varadhan. numDeriv: Accurate Numerical Derivatives, 2016. URL https://CRAN.R-project.org/package=numDeriv. R package version 2016.8-1. 
H. Glejser. A new test for heteroscedasticity. Journal of the American Statistical Association, 64(325):316-323, March 1969.

Stephen M. Goldfeld and Richard E. Quandt. Some tests for homoscedasticity. Journal of the American Statistical Association, 60(310):539-547, 1965. doi: 10. 1080/01621459.1965.10480811. URL http://www.tandfonline.com/doi/abs/10.1080/ 01621459.1965 .10480811$.

H. O. Hartley. The maximum f-ratio as a short cut test for homogeneity of variance. Biometrika, 37(3/4):308-312, 1950.

Andrew F. Hayes and Li Cai. Using heteroskedasticity-consistent standard error estimators in ols regression: An introduction and software implementation. Behavior Research Methods, 39(4):709-722, 2007.

Clifford Hildreth and James P. Houck. Some estimators for a linear model with random coefficients. Journal of the American Statistical Association, 63(322):584-595, June 1968.

Jennifer A. Hoeting, David Madigan, Adrian E. Raftery, and Chris T. Volinsky. Bayesian model averaging: a tutorial (with comments by m. clyde, david draper and e. i. george, and a rejoinder by the authors. Statist. Sci., 14(4):382-417, 11 1999. doi: 10.1214/ss/ 1009212519. URL https://doi.org/10.1214/ss/1009212519.

Peter J. Huber. The behavior of maximum likelihood estimates under nonstandard conditions. In Proceedings of the Fifth Berkeley Symposium on Mathematical Statistics and Probability, Volume 1: Statistics, pages 221-233, Berkeley, Calif., 1967. University of California Press. URL https://projecteuclid.org/euclid.bsmsp/1200512988.

J. D. Jobson and W. A. Fuller. Least squares estimation when the covariance matrix and parameter vector are functionally relate. Journal of the American Statistical Association, 75:176-181, 1980. 
M. Kharrati-Kopaei and S. M. Sadooghi-Alvandi. A new method for testing interaction in unreplicated two-way analysis of variance. Communications in Statistics - Theory and Methods, 36(15):2787-2803, 2007. doi: 10.1080/03610920701386851. URL http: //dx.doi.org/10.1080/03610920701386851.

Howard Levene. Contributions to Probability and Statistics: Essays in Honor of Harold Hotelling, chapter Robust tests for equality of variances 1960, pages 278-292. Stanford University Press, 1960.

Feng Liang, Rui Paulo, German Molina, Merlise A Clyde, and Jim O Berger. Mixtures of g priors for bayesian variable selection. Journal of the American Statistical Association, 103(481):410-423, 2008. doi: 10.1198/016214507000001337. URL https://doi.org/10. $1198 / 016214507000001337$.

J. Scott Long and Laurie H. Ervin. Using heteroscedasticity consistent standard errors in the linear regression model. The American Statistician, 54(3):217-224, 2000. doi: 10.1080/00031305.2000.10474549. URL https://www.tandfonline.com/doi/abs/10. $1080 / 00031305.2000 .10474549$.

James G MacKinnon and Halbert White. Some heteroskedasticity-consistent covariance matrix estimators with improved finite sample properties. Journal of Econometrics, 29 (3):305 - 325, 1985. ISSN 0304-4076. doi: https://doi.org/10.1016/0304-4076(85)90158-7. URL http://www. sciencedirect.com/science/article/pii/0304407685901587.

G.E. Meek and C.O. Ozgur. Torque variation analysis. Journal of the Industrial Mathematics Society, 41:1-16, 1991.

Thomas A. Metzger and Christopher T. Franck. Detection of latent heteroscedasticity and group-based regression effects in linear models via Bayesian model selection. arXiv eprints, art. arXiv:1903.01035, Mar 2019.

Donald F. Morrison. Applied Linear Statistical Methods. Prentice-Hall, 1983. 
R. G. O'Brien and M. W. Heft. New discrimination indexes and models for studying sensory functioning in aging. Journal of Applied Statistics, 22:9-27, 1995.

Anthony O'Hagan. Fractional bayes factors for model comparison. Journal of the Royal Statistical Society. Series B (Methodological), 57(1):99-138, 1995. ISSN 00359246. URL http ://www . jstor.org/stable/2346088.

R. E. Park. Estimation with heteroscedastic error terms. Econometrica, 34(4):888, 1966.

Frieder. Andreas Perthes. Carolus fridericus gauss theoria motus corporum coelestium in sectionibus conicis solem ambientium. Astronomische Nachrichten, 40(25):391-392, 1855. ISSN 1521-3994. doi: 10.1002/asna.18550402508. URL http://dx.doi.org/10.1002/ asna. 18550402508.

W. Polasek, S. Liu, and S. Jin. Heteroskedastic linear regression models a bayesian analysis. In Ingo Balderjahn, Rudolf Mathar, and Martin Schader, editors, Classification, Data Analysis, and Data Highways, pages 182-191, Berlin, Heidelberg, 1998. Springer Berlin Heidelberg. ISBN 978-3-642-72087-1.

Wolfgang Polasek and Klaus Pötzelberger. Robust bayesian methods in simple anova models. Journal of Statistical Planning and Inference, 40(2):295 - 311, 1994. ISSN 0378-3758. doi: https://doi.org/10.1016/0378-3758(94)90127-9. URL http://www. sciencedirect.com/ science/article/pii/0378375894901279.

R Core Team. R: A Language and Environment for Statistical Computing. R Foundation for Statistical Computing, Vienna, Austria, 2017. URL https://www.R-project.org/.

Potluri Rao. Some notes on misspecification in multiple regressions. The American Statistician, 25(5):37-39, December 1971.

Alvin C. Rencher and G. Bruce Schaalje. Linear Models in Statistics. John Wiley and Sons, 2nd edition, 2008. ISBN 978-0-471-75498-5.

Henry Scheffé. The Analysis of Variance. Wiley Publications in Statistics, 1959. 
John W. Tukey. Comparing individual means in the analysis of variance. Biometrics, 5(2): 99-114, 1949. ISSN 0006341X, 15410420. URL http://www. jstor.org/stable/3001913.

B. L. Welch. On the comparison of several mean values: An alternative approach. Biometrika, 38(3/4):330-336, 1951. ISSN 00063444. URL http://www.jstor.org/stable/2332579.

Halbert White. A heteroskedasticity-consistent covariance matrix estimator and a direct test for heteroskedasticity. Econometrica, 48(4):817-838, May 1980.

A. Zellner and A. Siow. Posterior odds ratios for selected regression hypotheses. Trabajos de Estadistica y de Investigacion Operativa, 31(1):585-603, Feb 1980. ISSN 0041-0241. doi: 10.1007/BF02888369. URL https://doi.org/10.1007/BF02888369.

Arnold Zellner. On assessing prior distributions and bayesian regression analysis with g-prior distributions. In P. Goel and Arnold Zellner, editors, Bayesian Inference and Decision Techniques: Essays in Honor of Bruno de Finetti, pages 233-243. Elsevier Science Publishers, Inc., 1986.

Yichi Zhang, Siyu Tao, Wei Chen, and Daniel W Apley. A latent variable approach to gaussian process modeling with qualitative and quantitative factors. Technometrics, (just-accepted):1-19, 2019. 


\title{
Appendix A
}

\section{Appendix: lm.slgf Code}

\author{
lm.slgf <- function(df, response, lgf=NA, \\ usermodels, prior="flat", \\ het $=\operatorname{rep}(0$, length (usermodels $))$, \\ $\min$. levels $=1$ ) \{
}

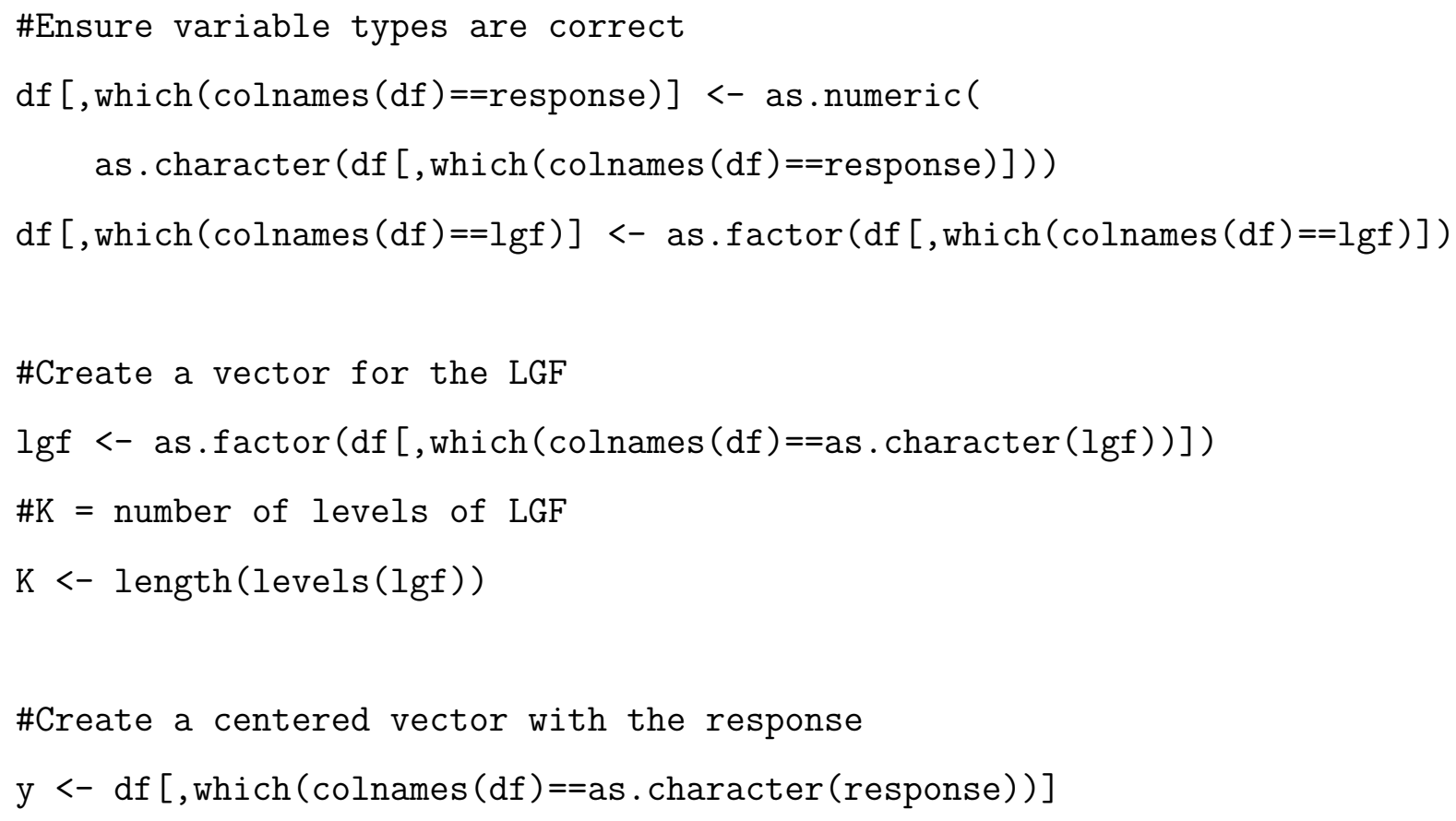




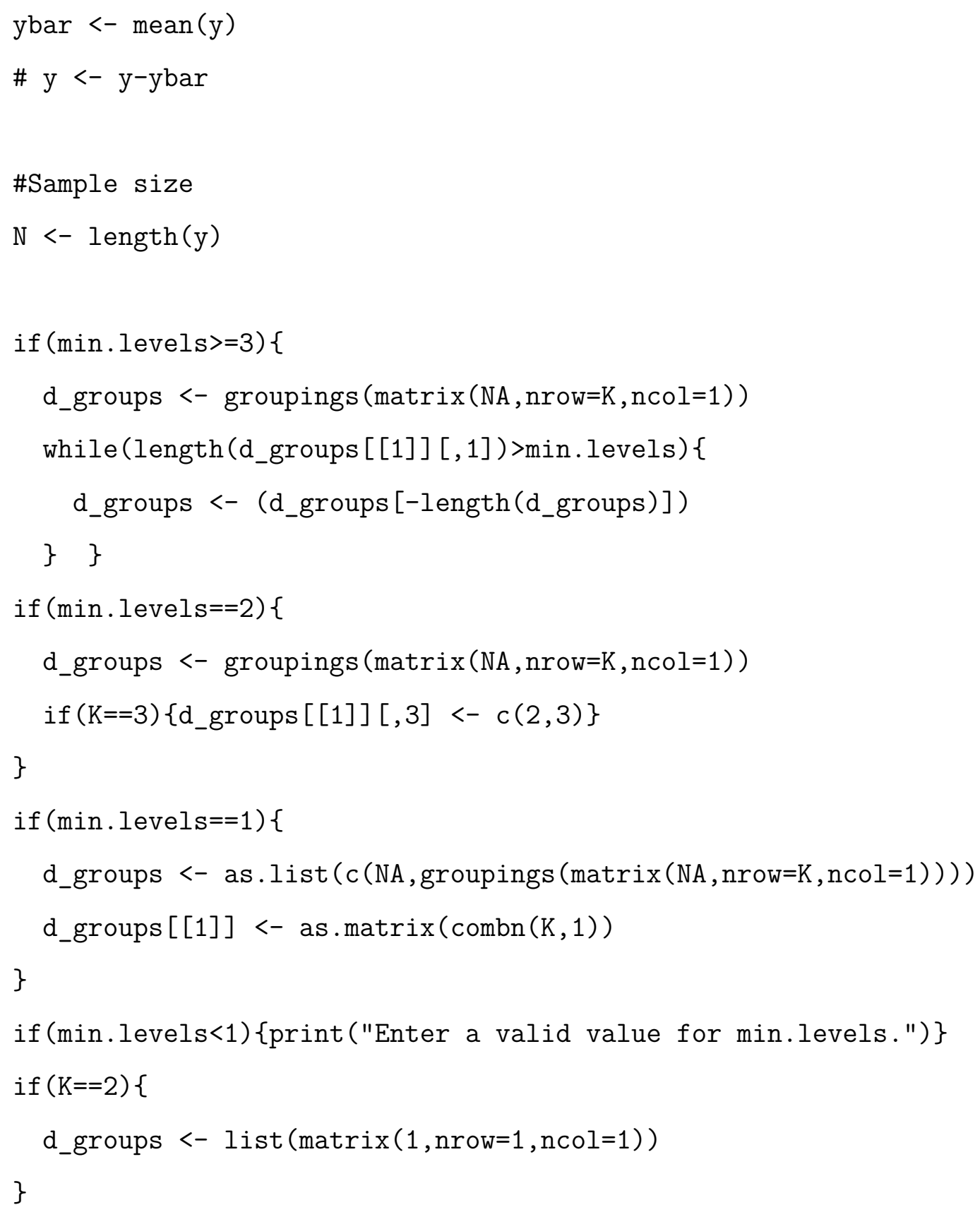




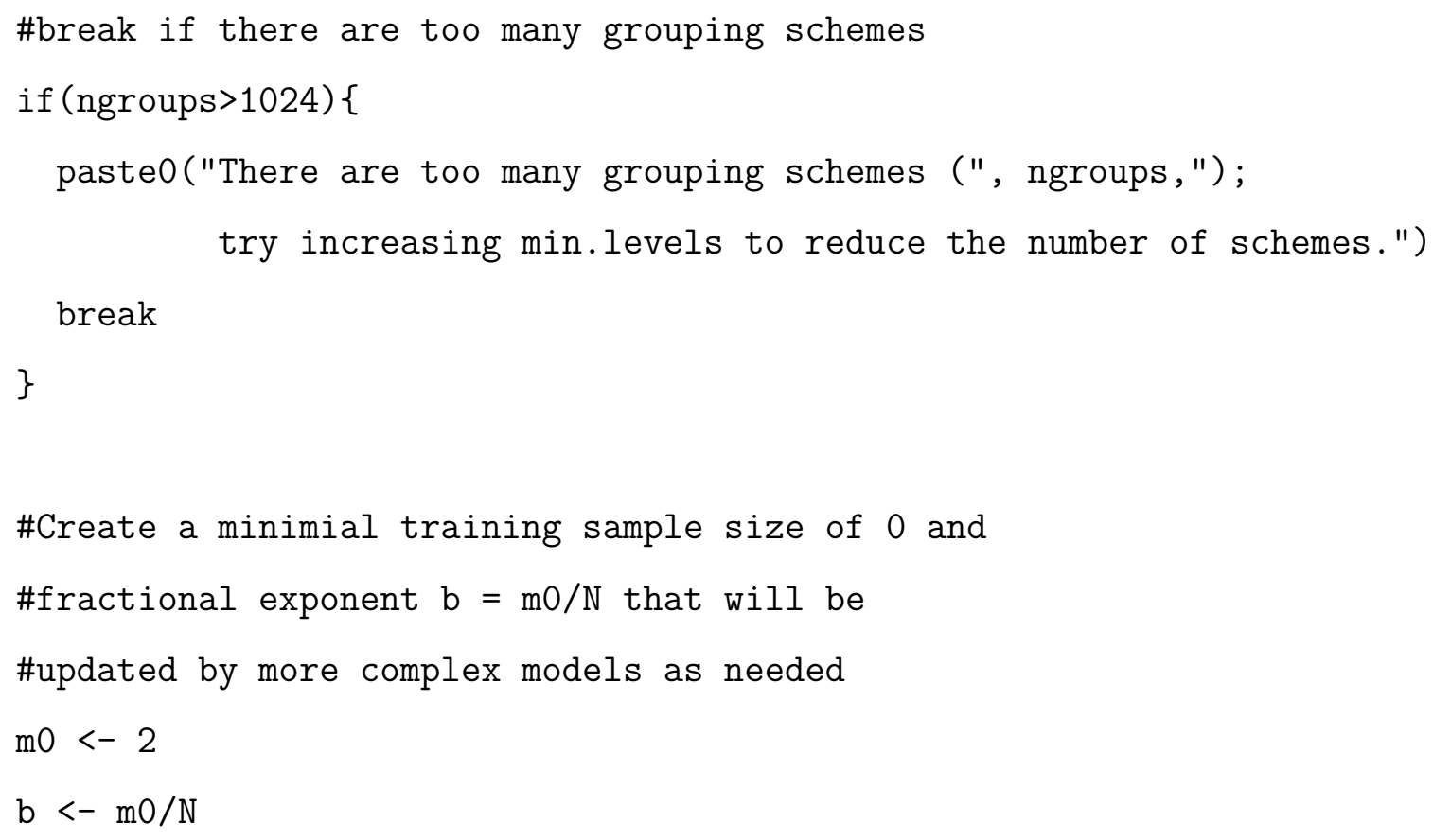

\#Determine which models do and do not contain a group-based fixed effect

index.lgf <- which(grepl("group", usermodels))

index.no.lgf <- which(! (1:length(usermodels)\%in\%index.lgf)) 


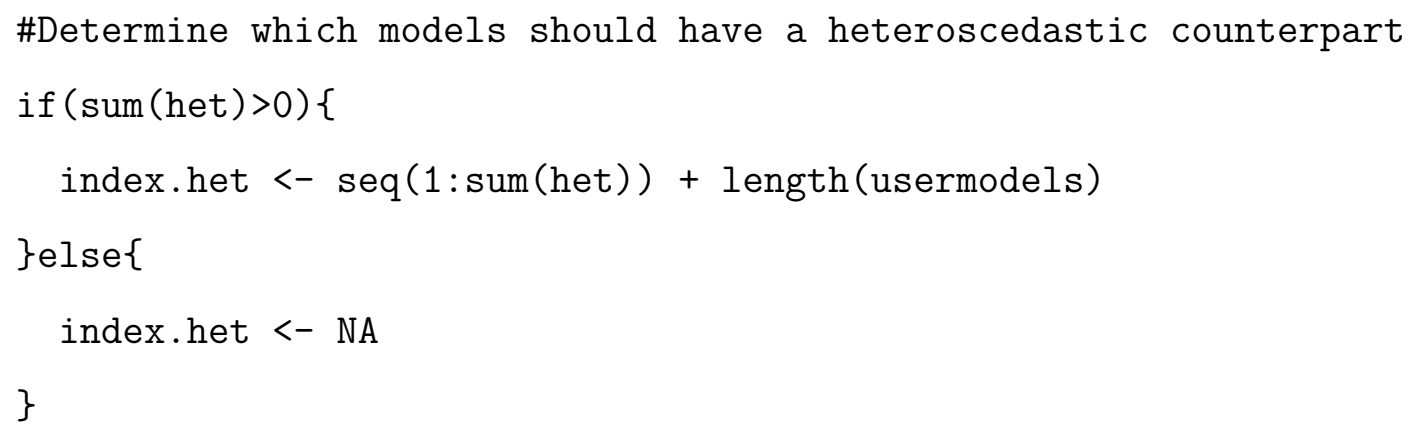




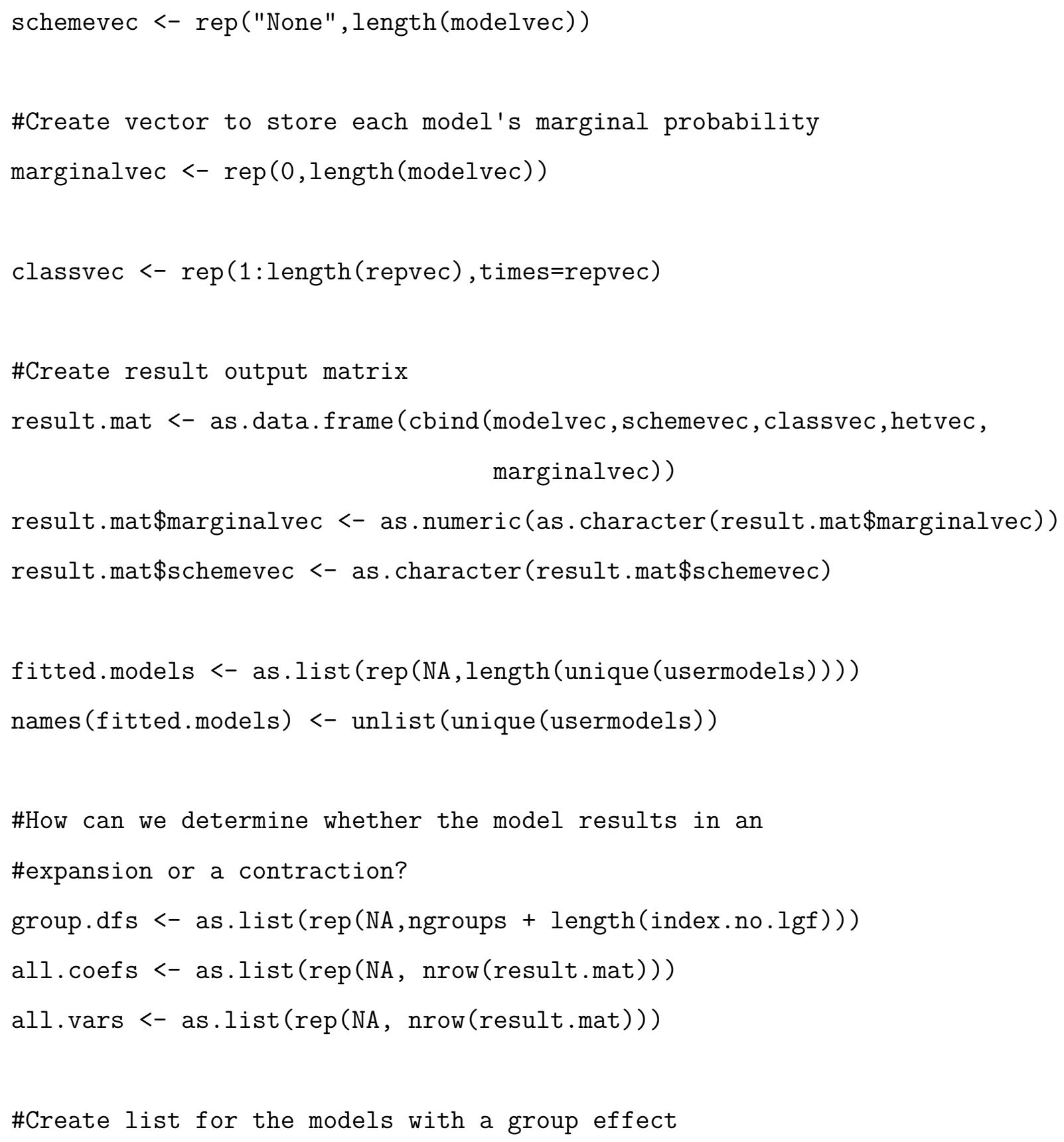




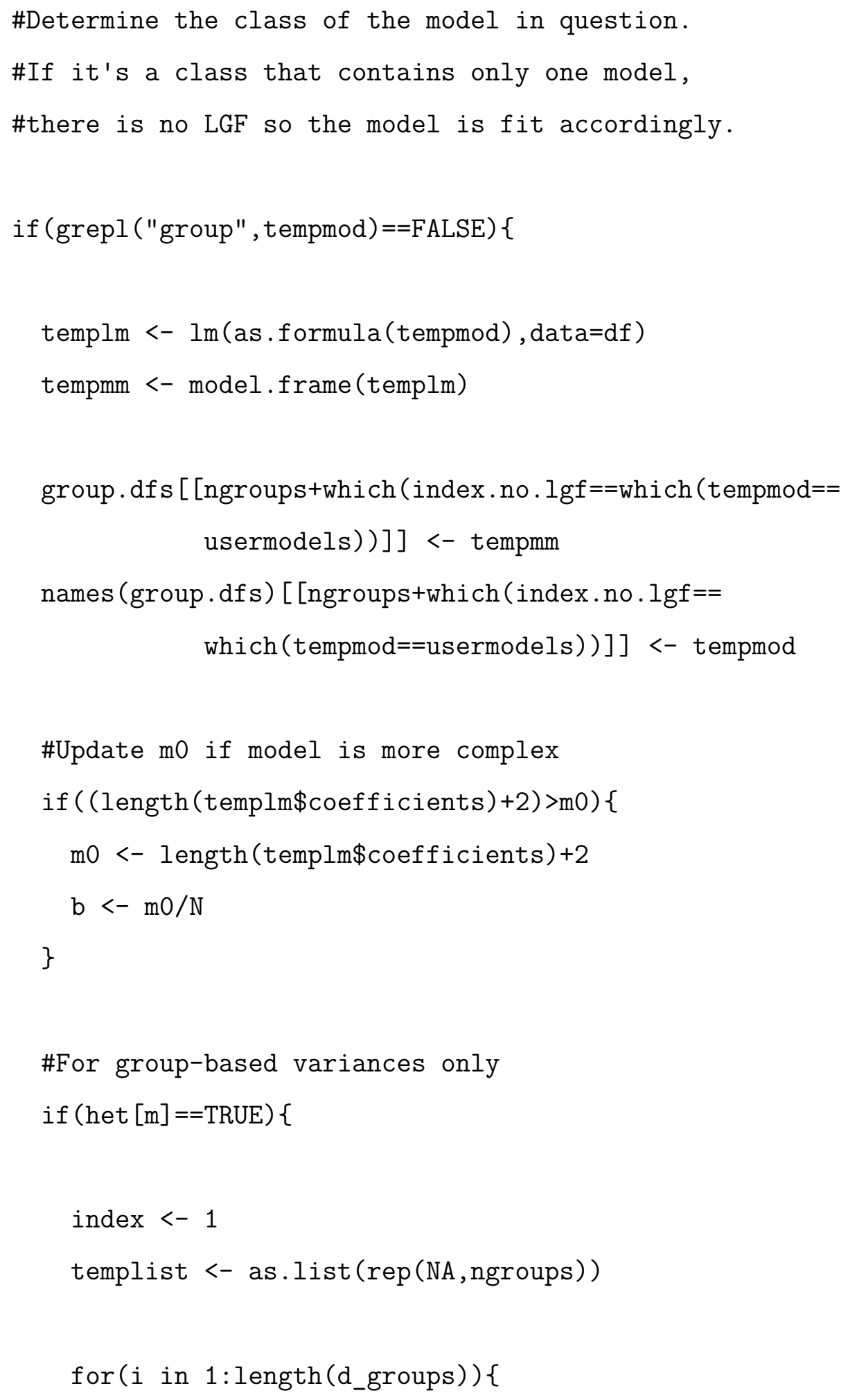




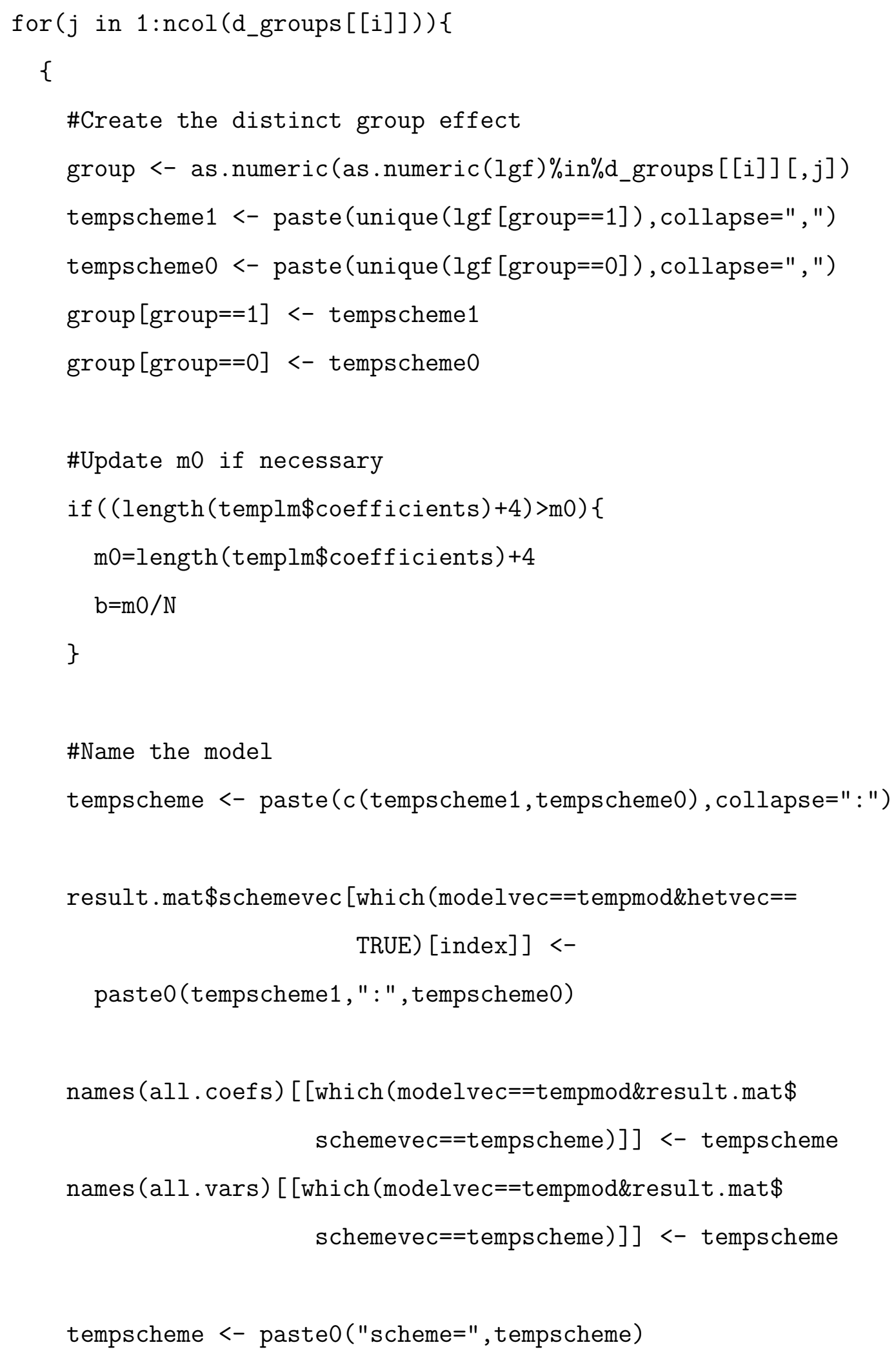




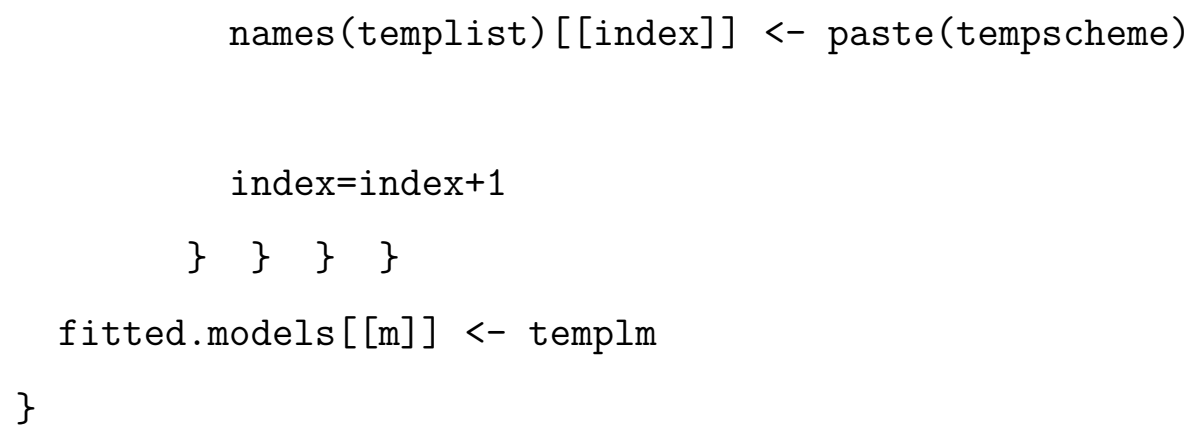




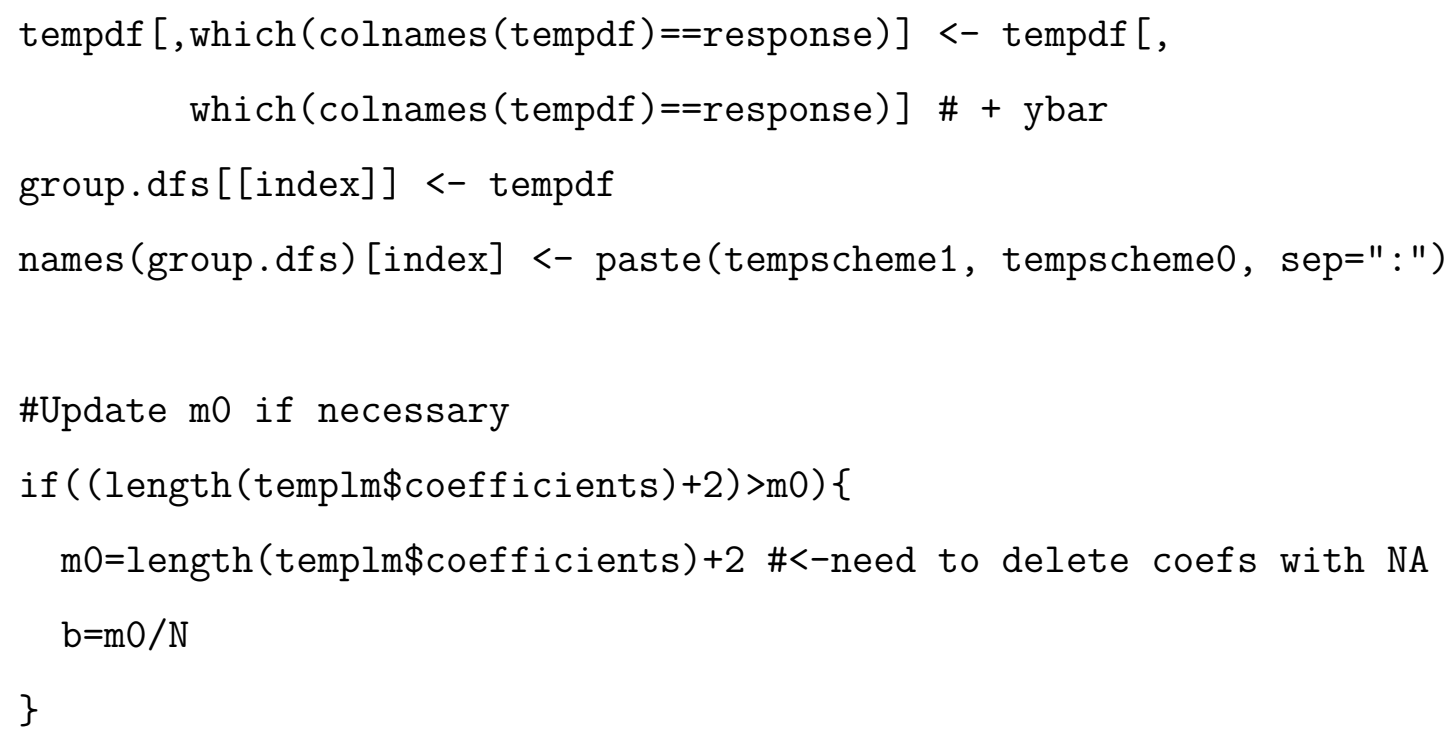




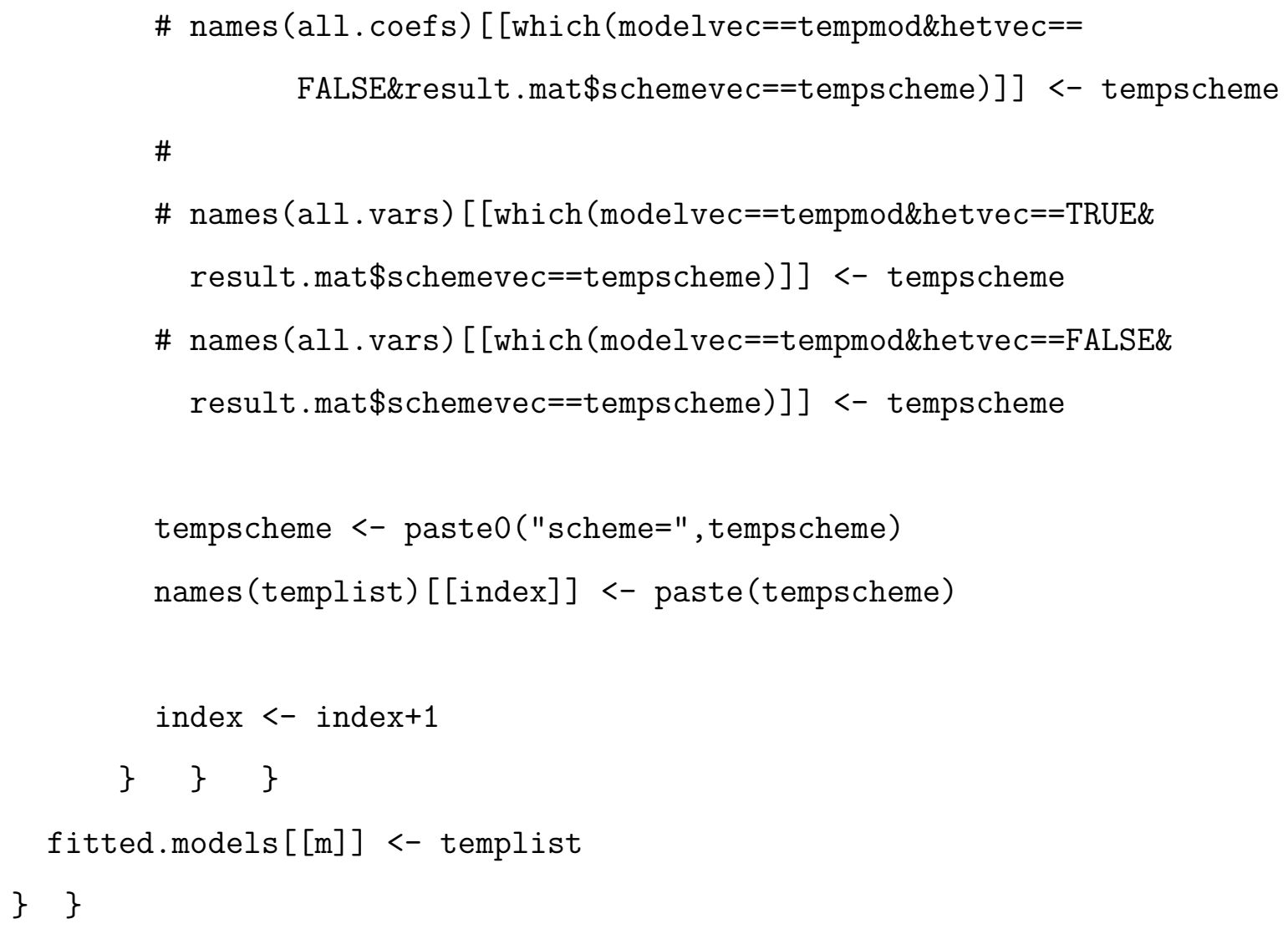




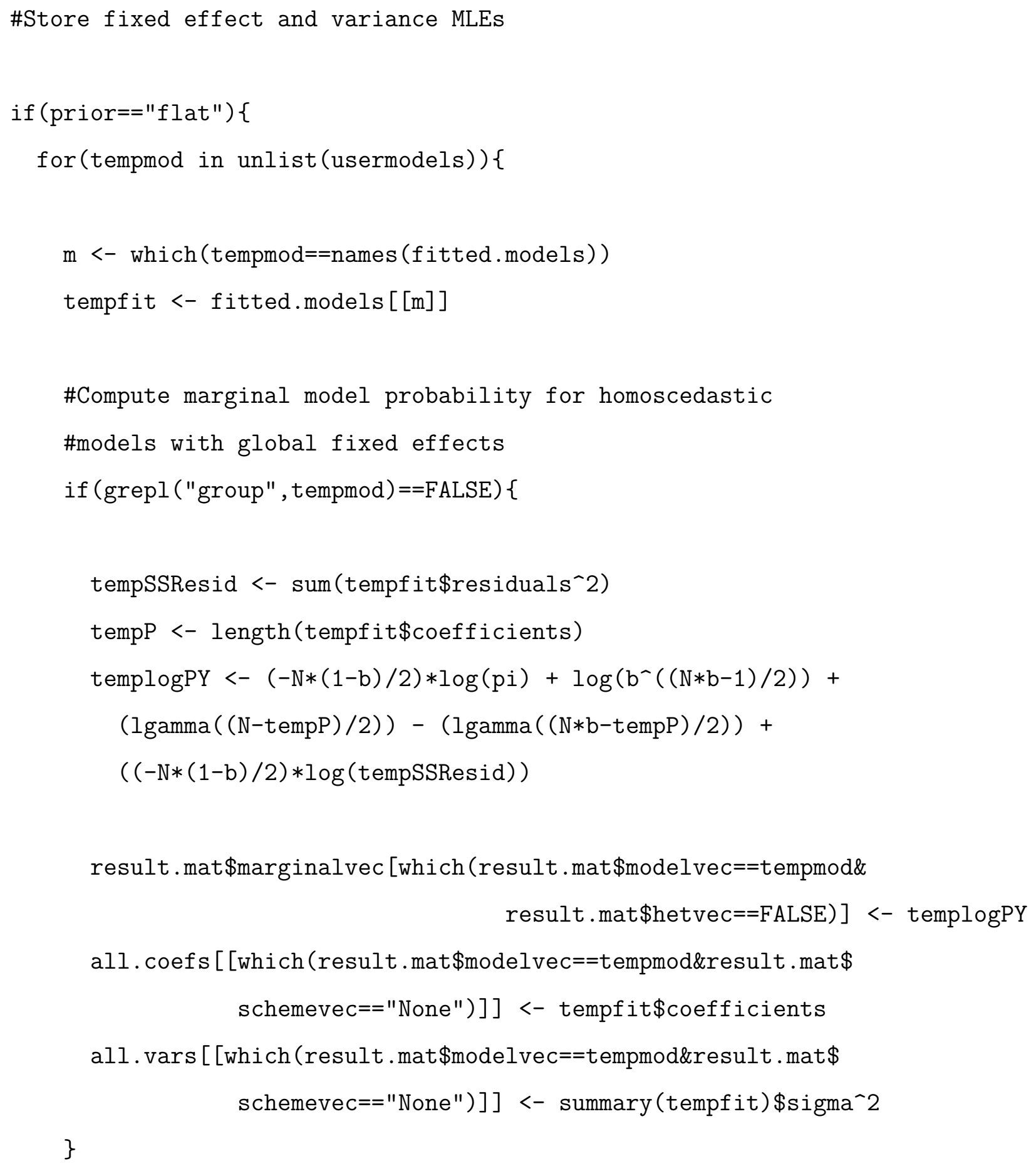




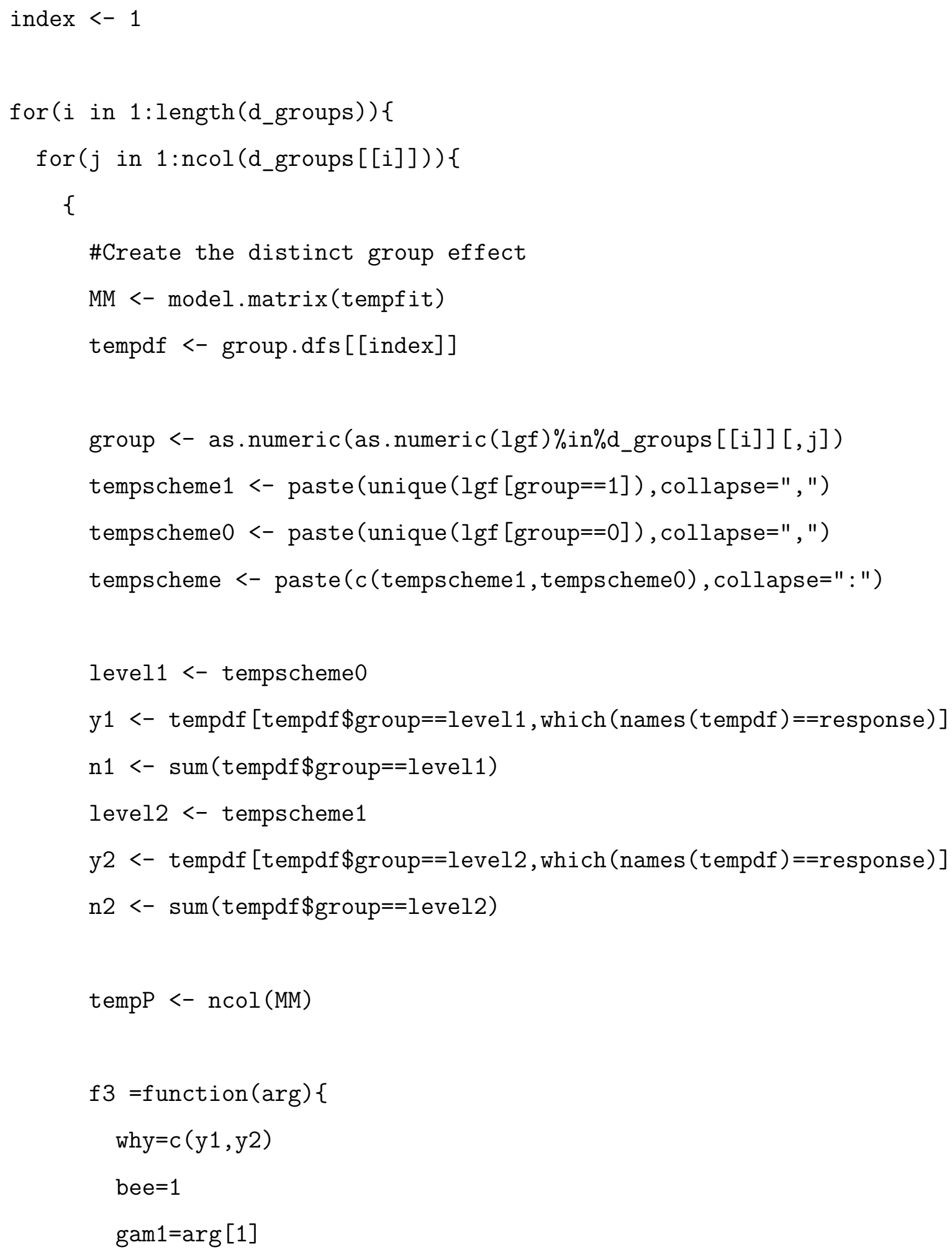




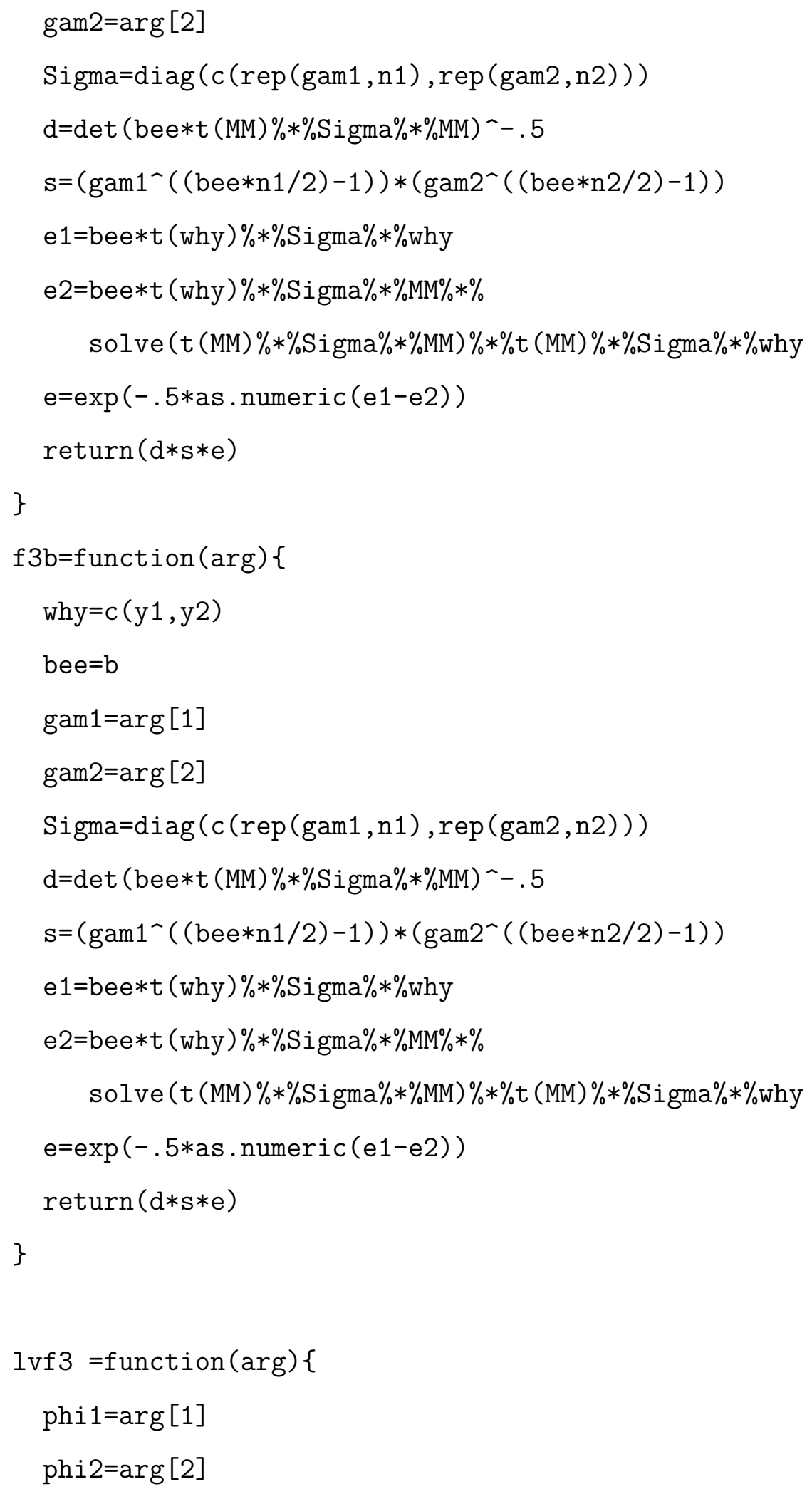




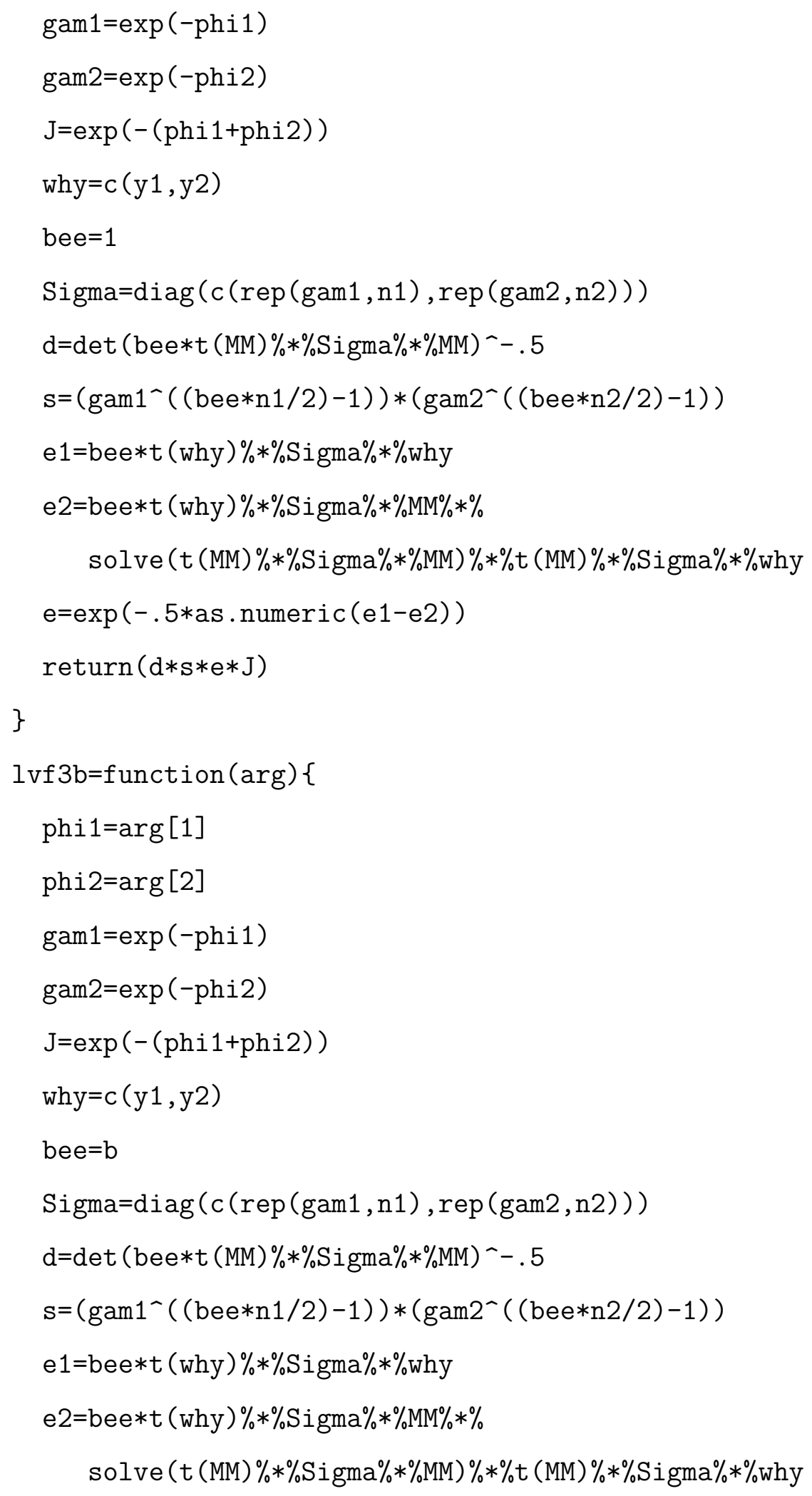




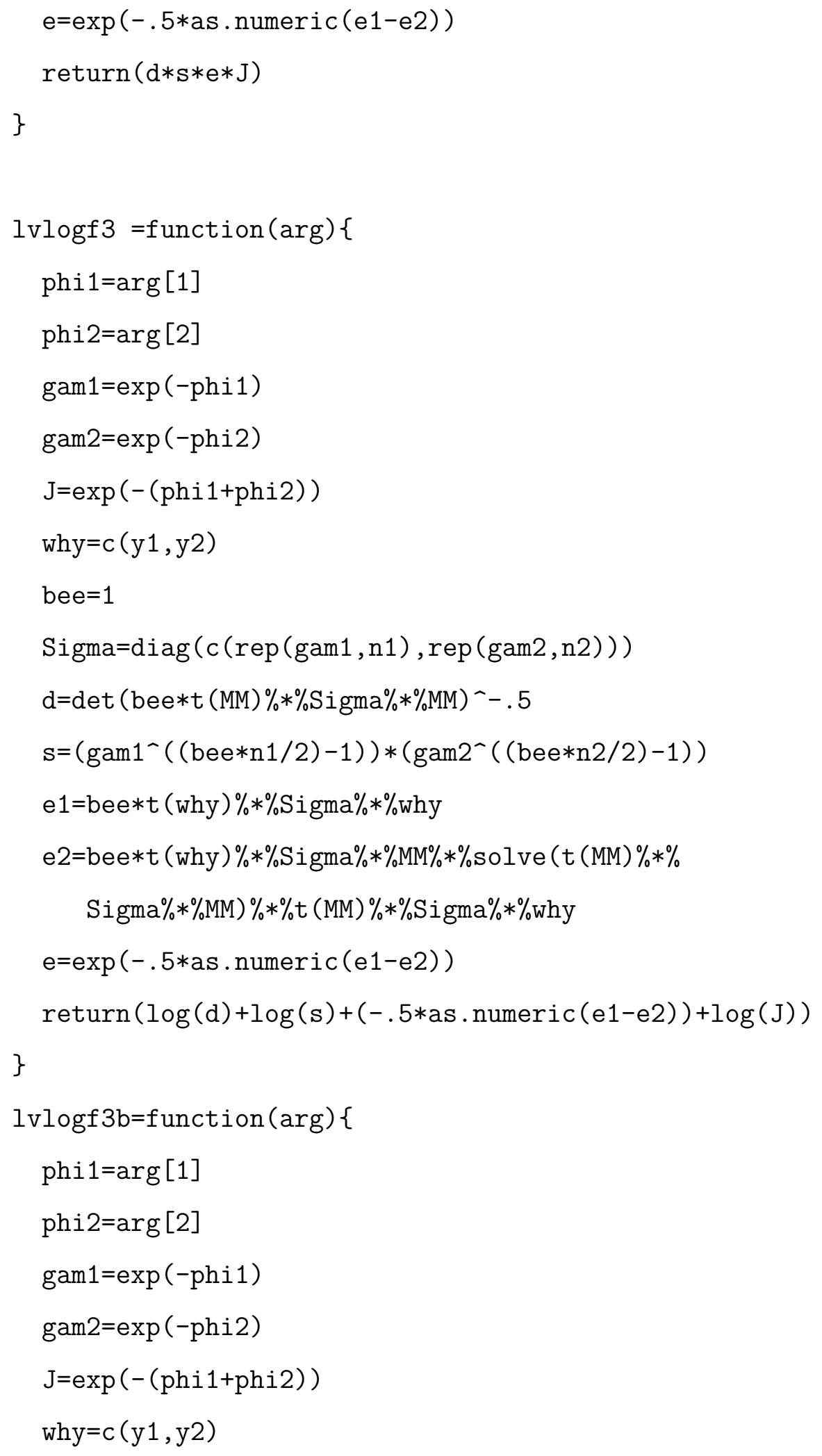




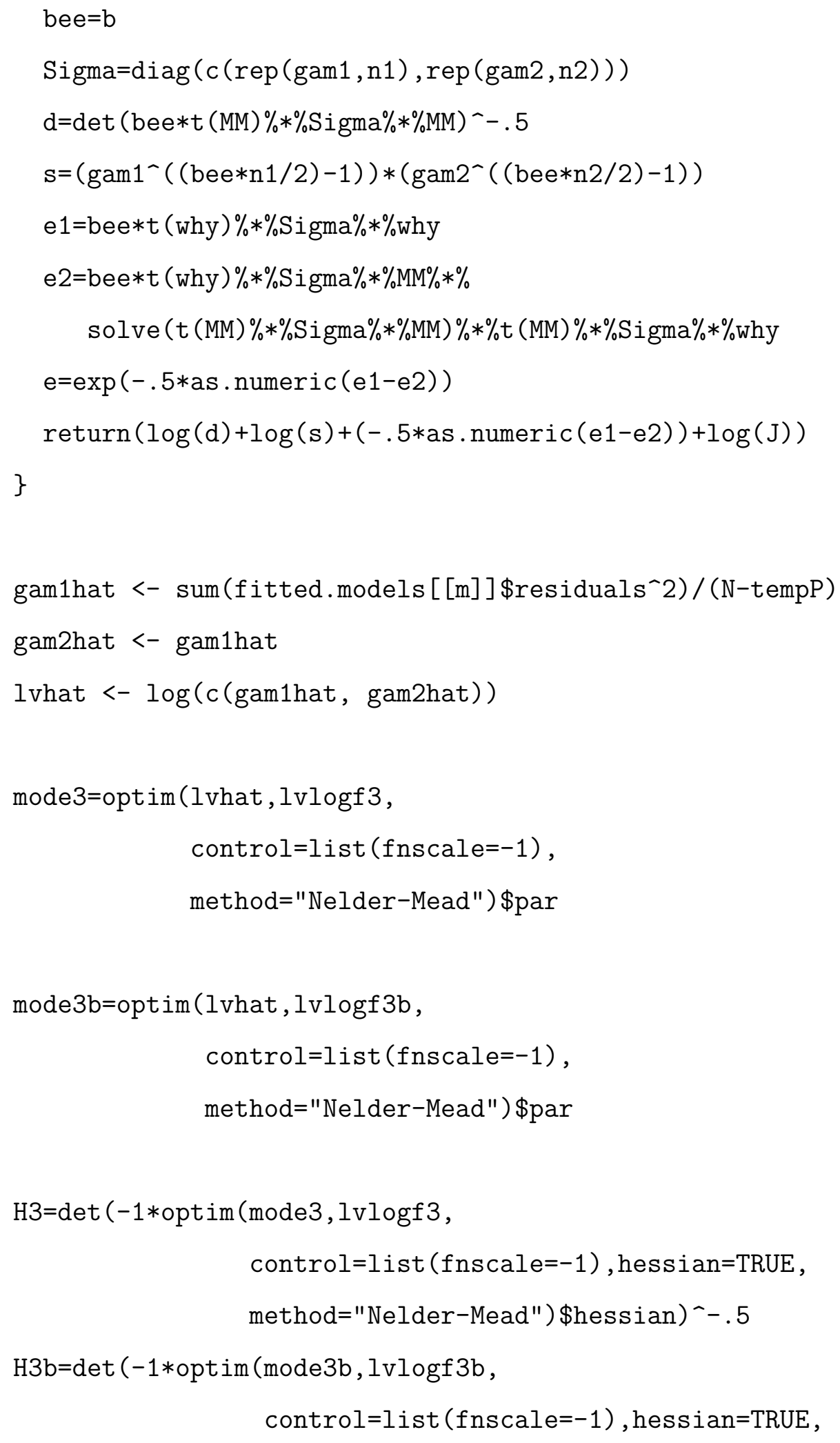




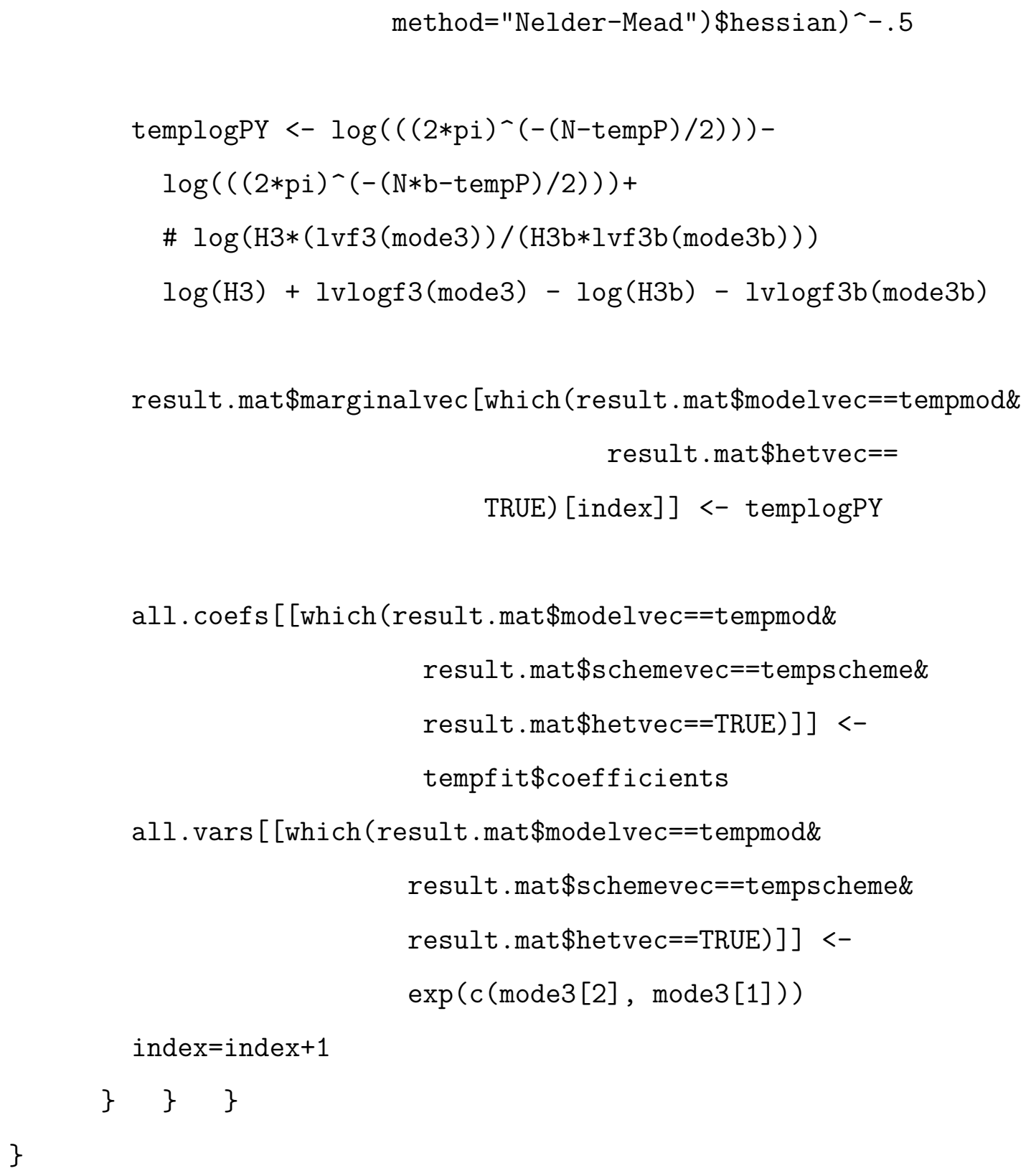




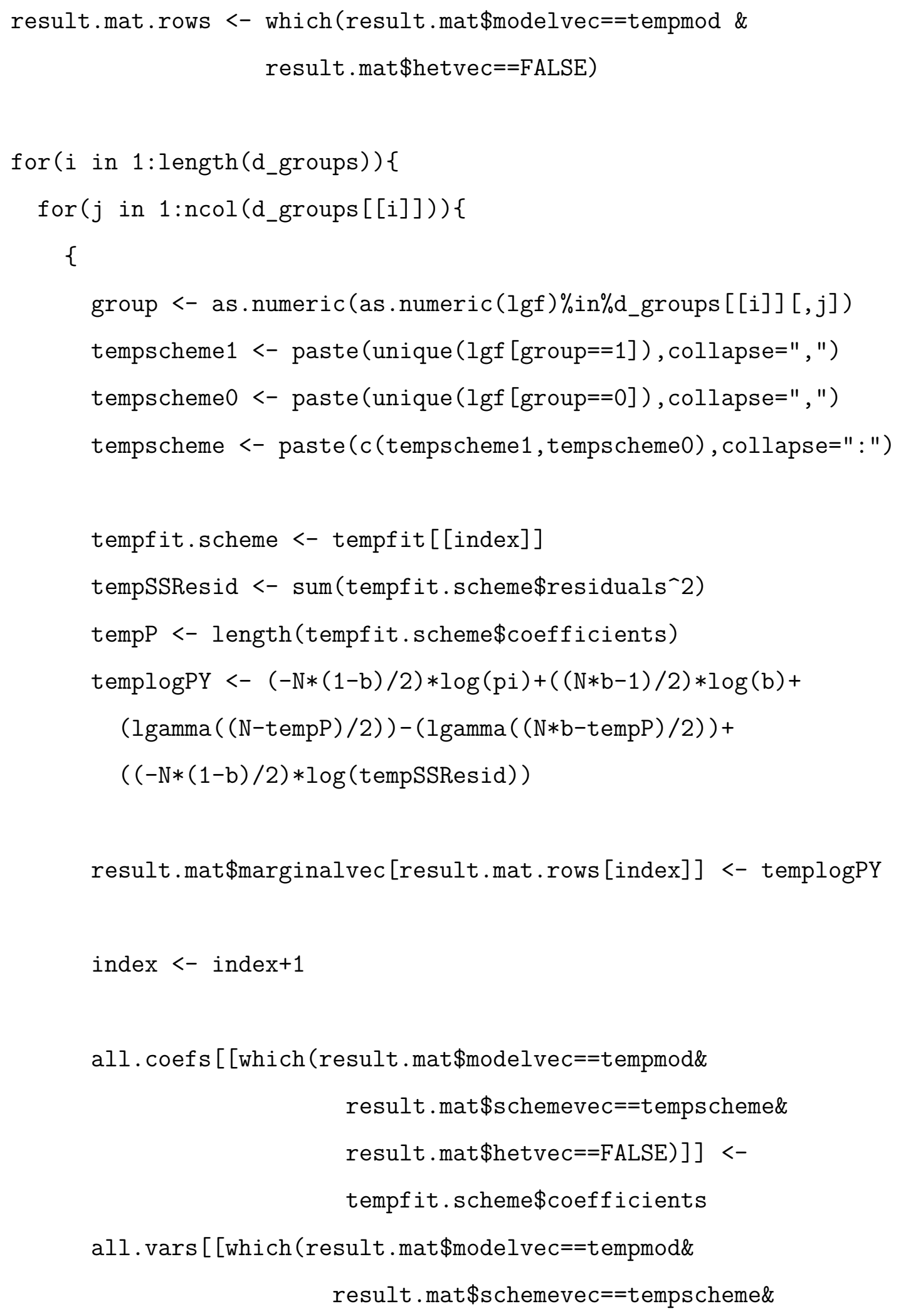




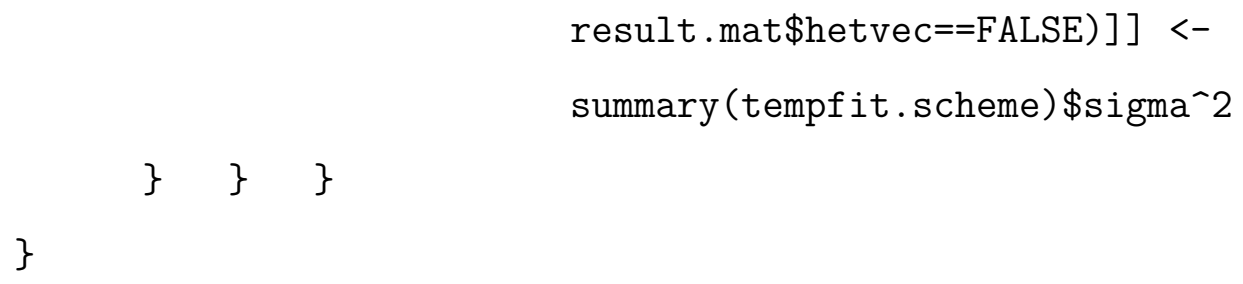




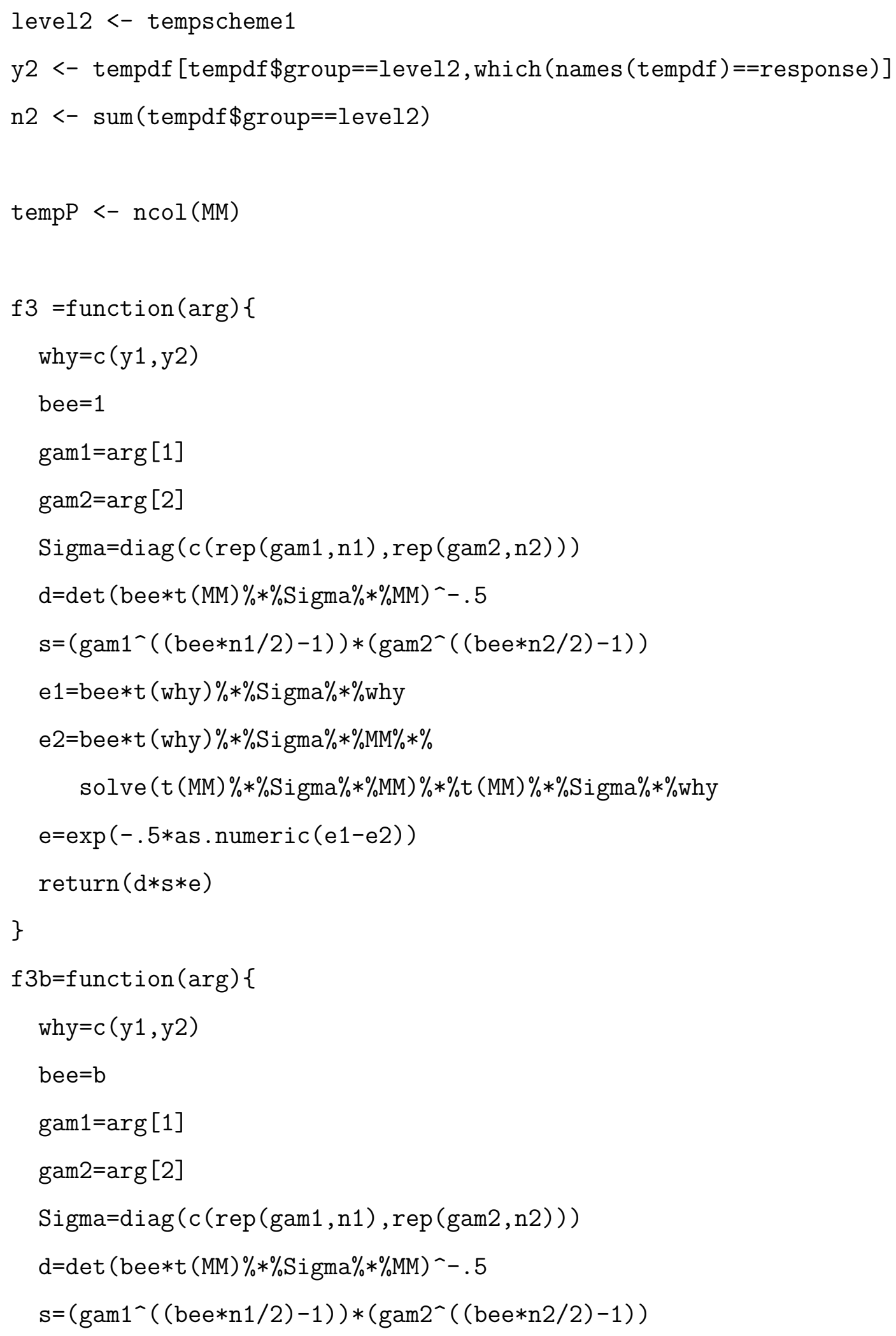




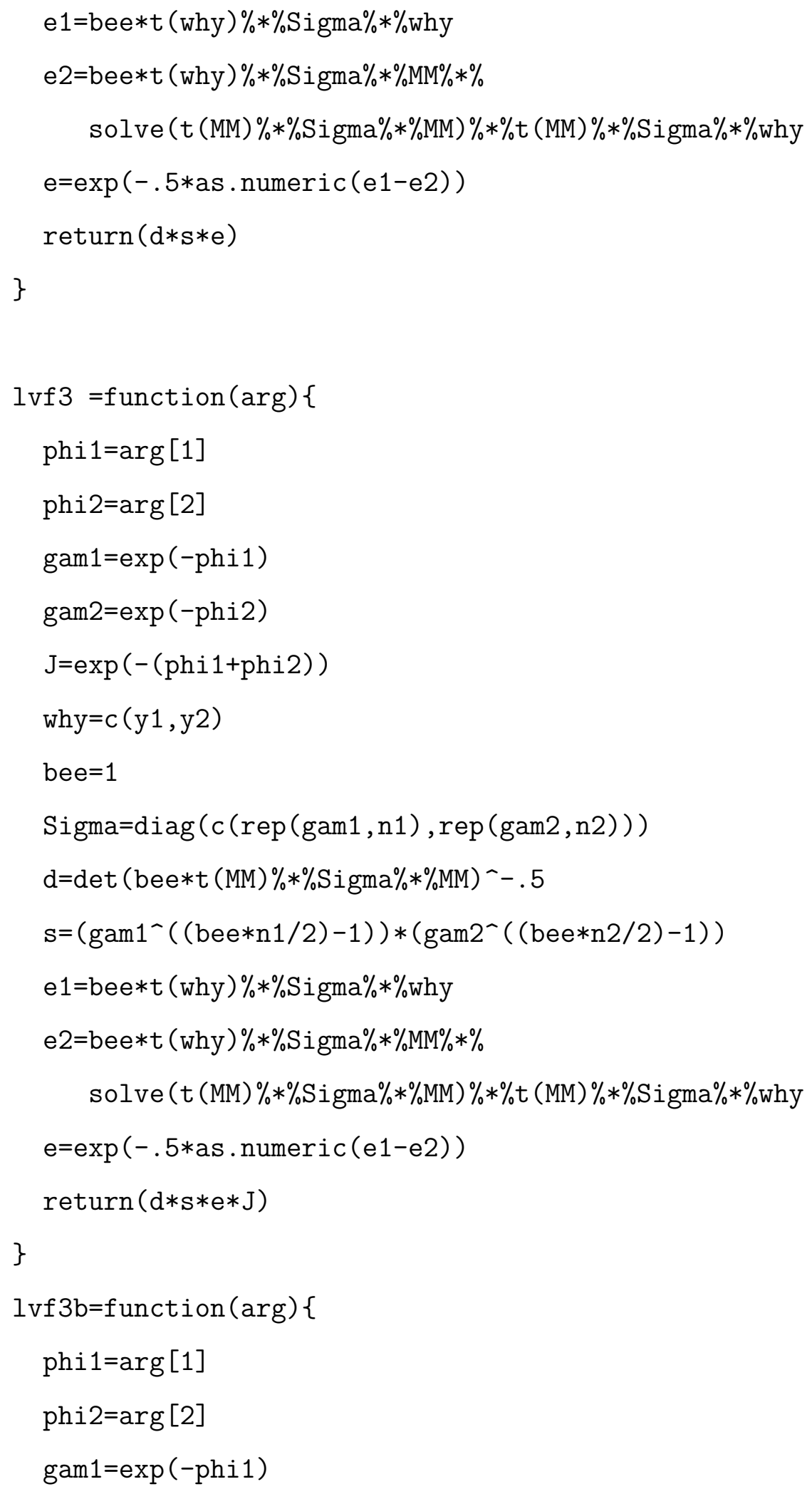




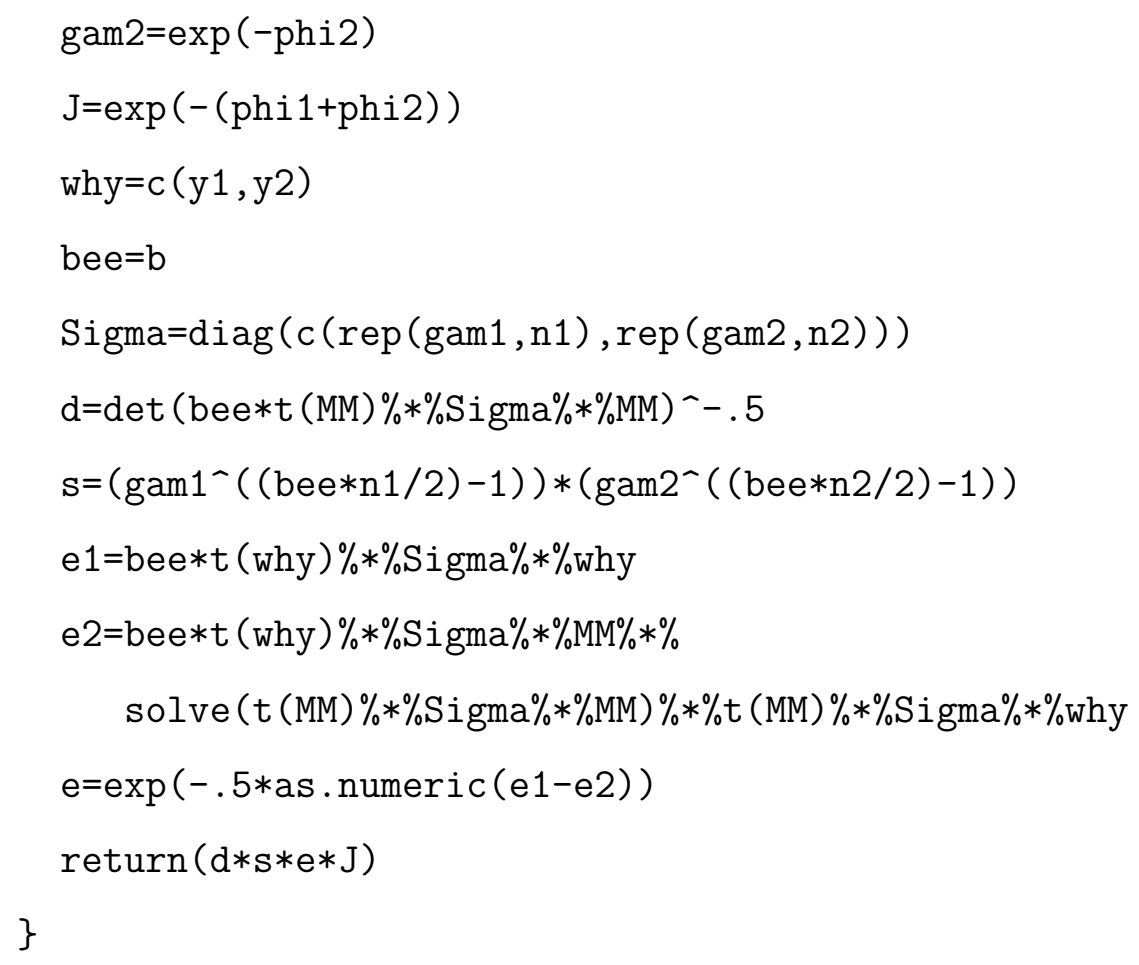




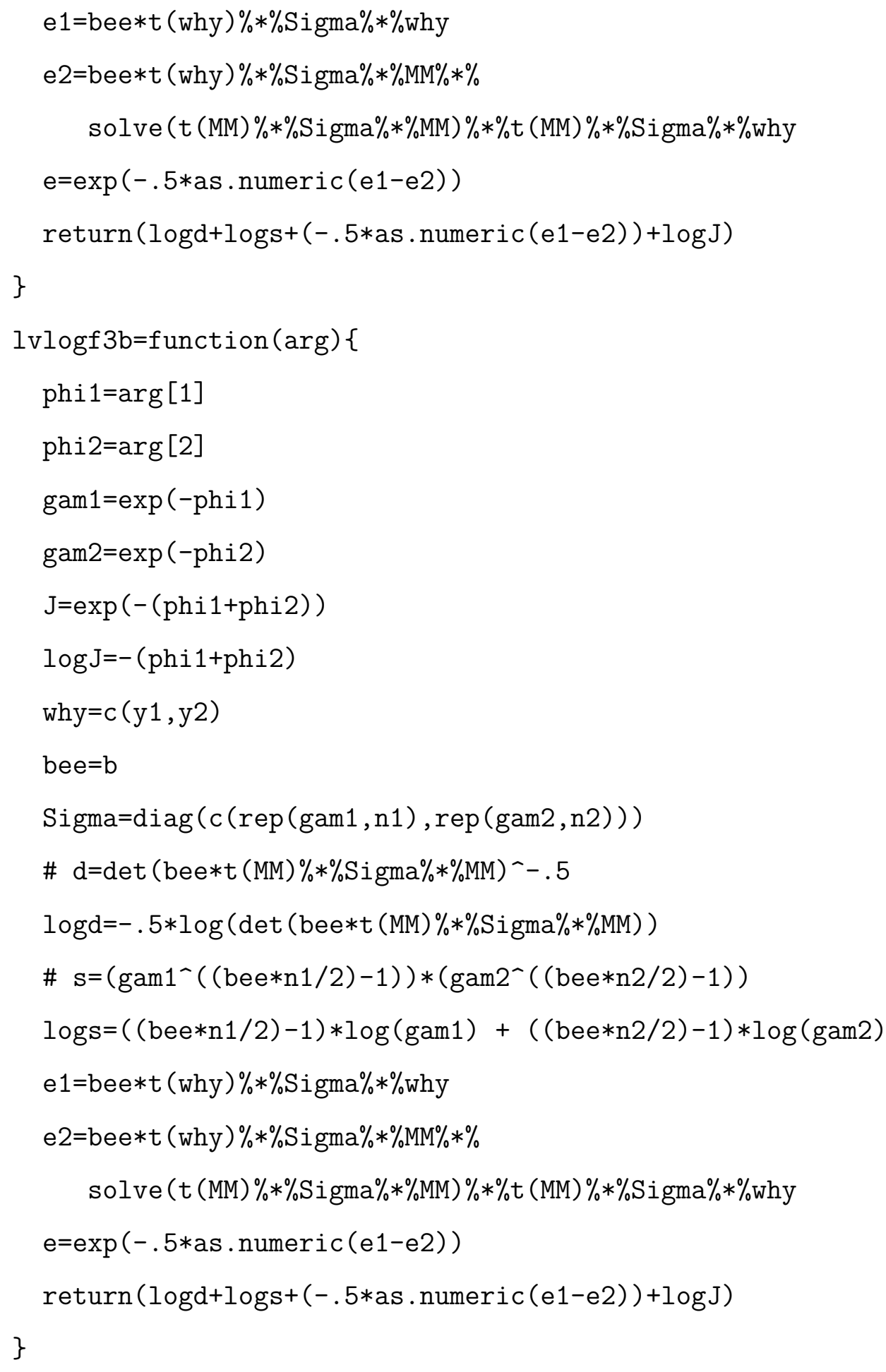




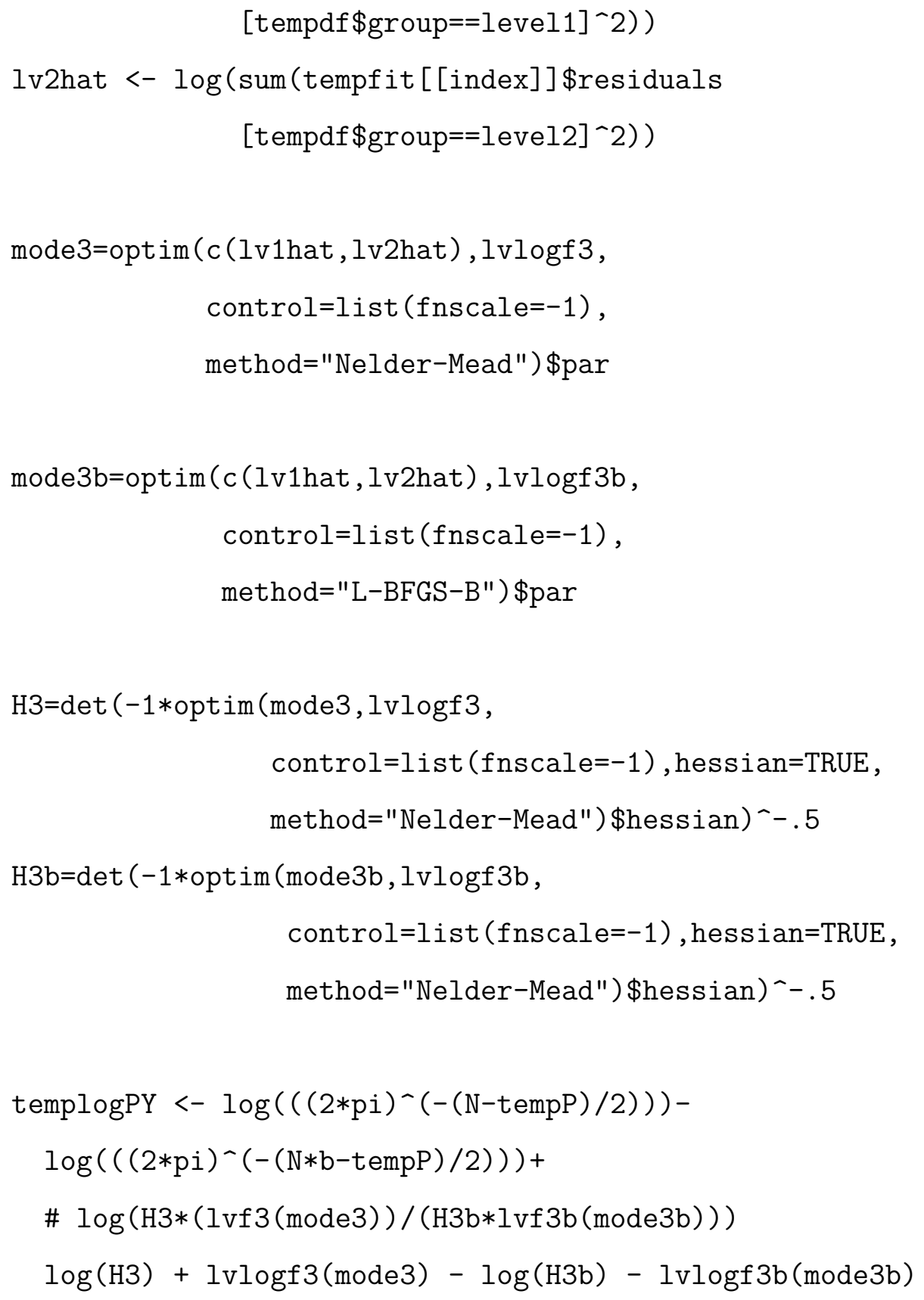


tempfit.scheme <- tempfit [ [index]

all . coef $\mathrm{s}[$ [which (result . mat $\$$ modelvec==tempmod\&

result. mat $\$$ s chemevec $==$ tempscheme\&

result . mat $\$$ hetvec $==$ TRUE) $]]<-$

tempfit.scheme\$coefficients

all. vars [ [which (result mat $\$$ modelvec==tempmod\&

result. mat $\$$ s chemevec $==$ temps cheme\&

result $\cdot$ mat $\$$ hetvec $==$ TRUE) ] ] <-

$\exp (c(\operatorname{mode} 3[2], \operatorname{mode} 3[1]))$

index $=$ index +1

\}$\}\}\}$

\}

\#total sum of squares

SST $<-\operatorname{sum}\left((y-\operatorname{mean}(\mathrm{y}))^{-} 2\right)$

$Z<-\operatorname{rep}(1, N)$

\#Compute marginal model probabilities - Zellner-Siow mixture g-prior case

if (prior $==" z s ")\{$

for(tempmod in unlist(usermodels)) \{

$\mathrm{m}<-$ which (tempmod==names (fitted.models))

tempfit <- fitted.models $[\mathrm{m}]]$

\#done $3 / 23$ 


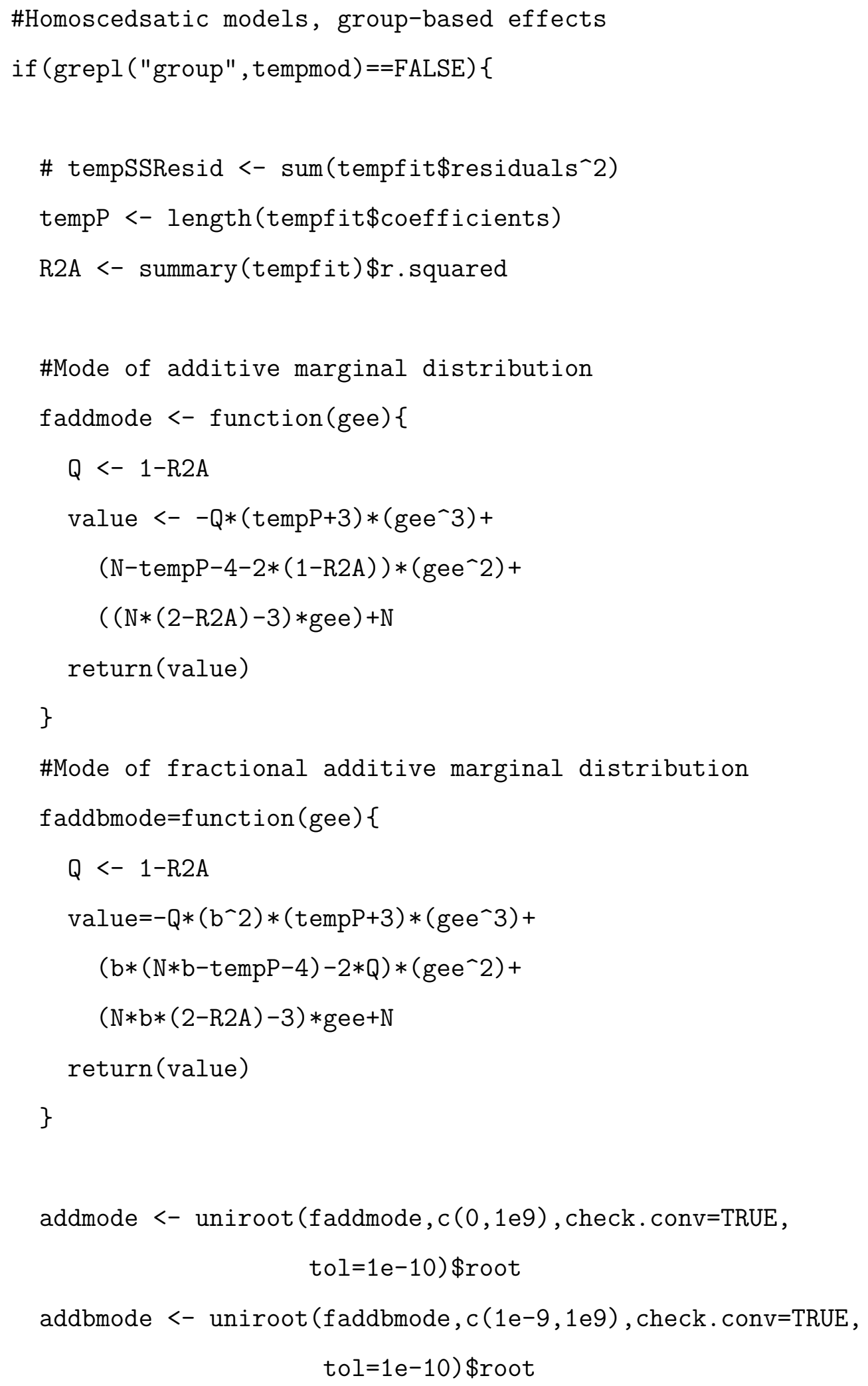




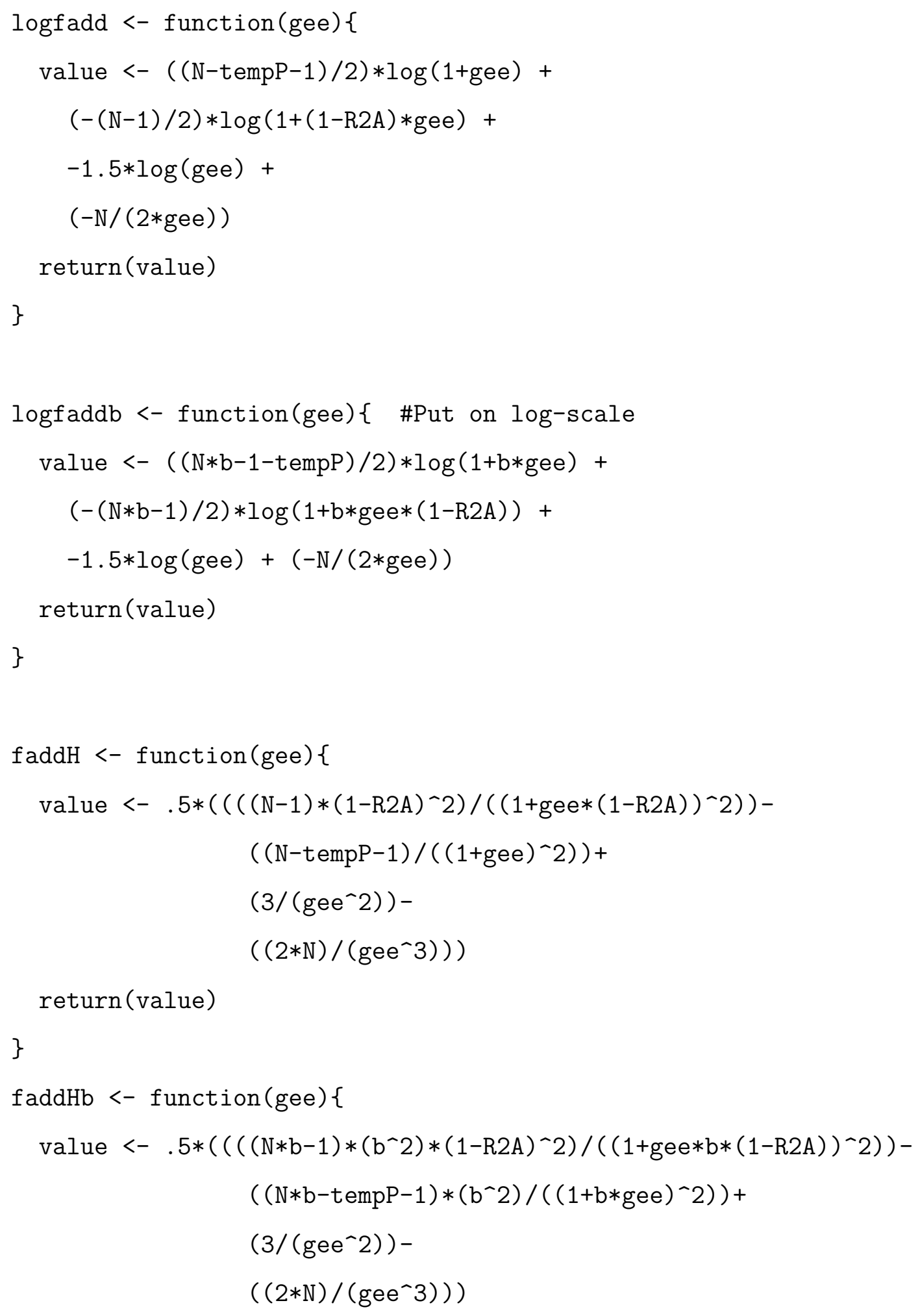




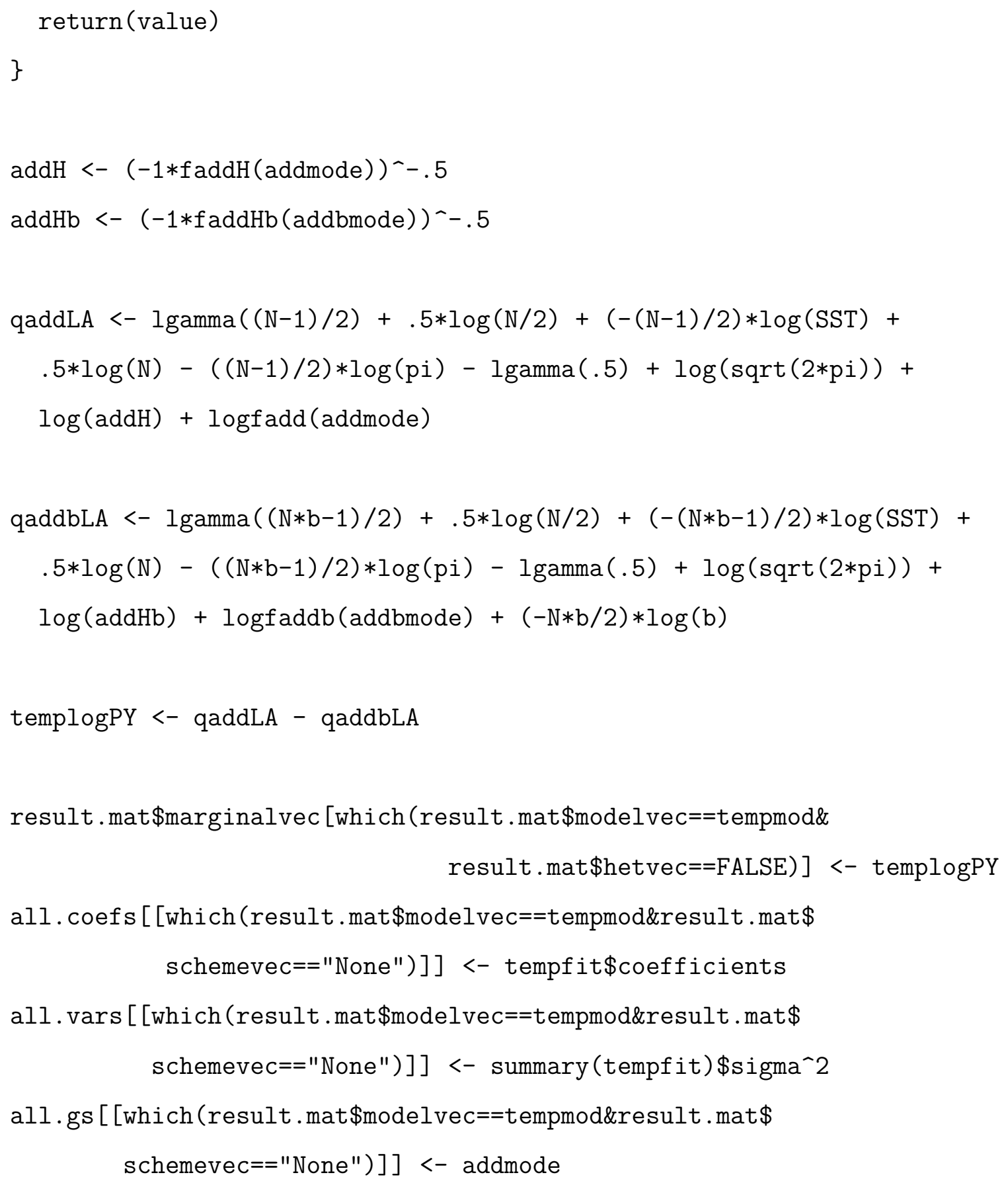




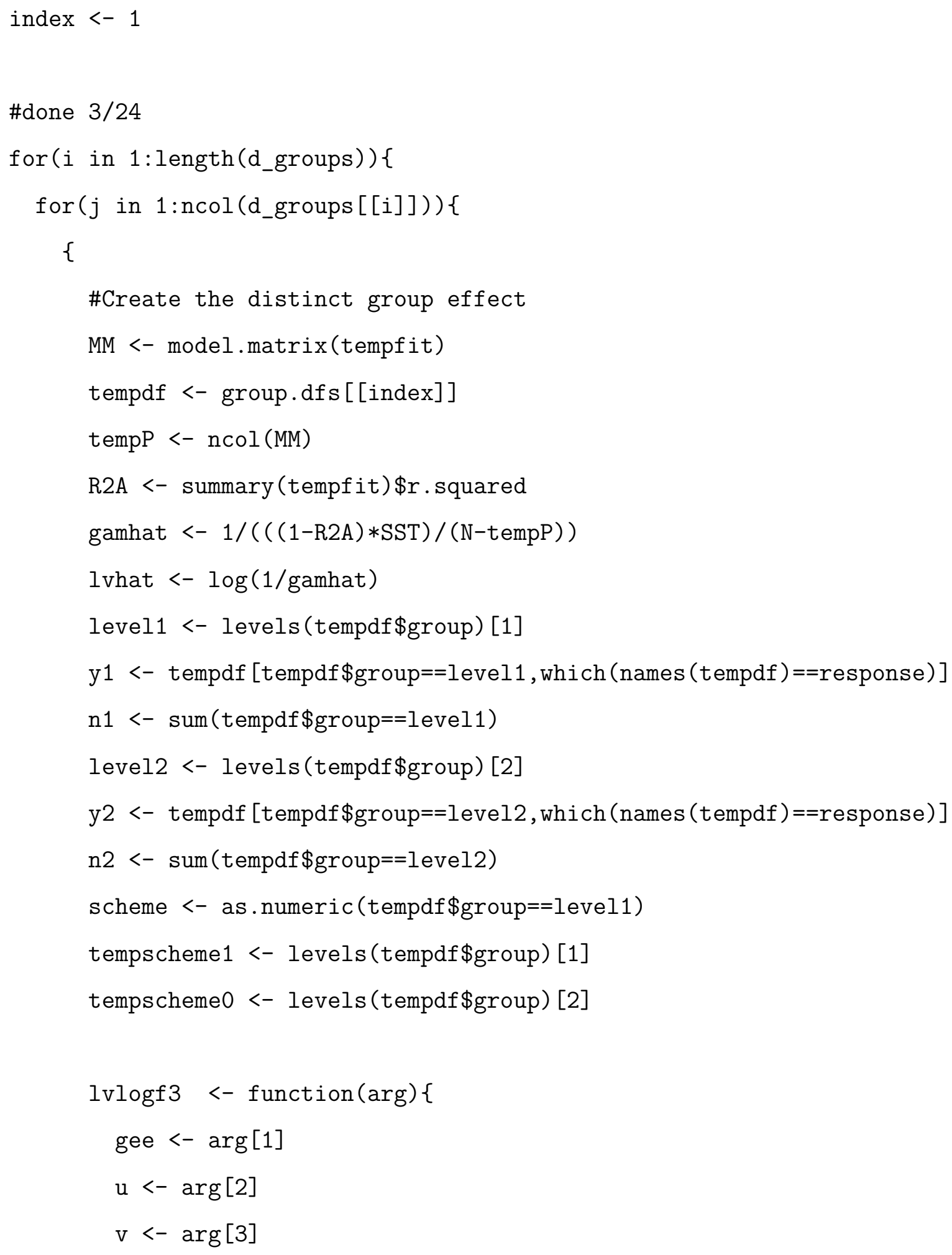




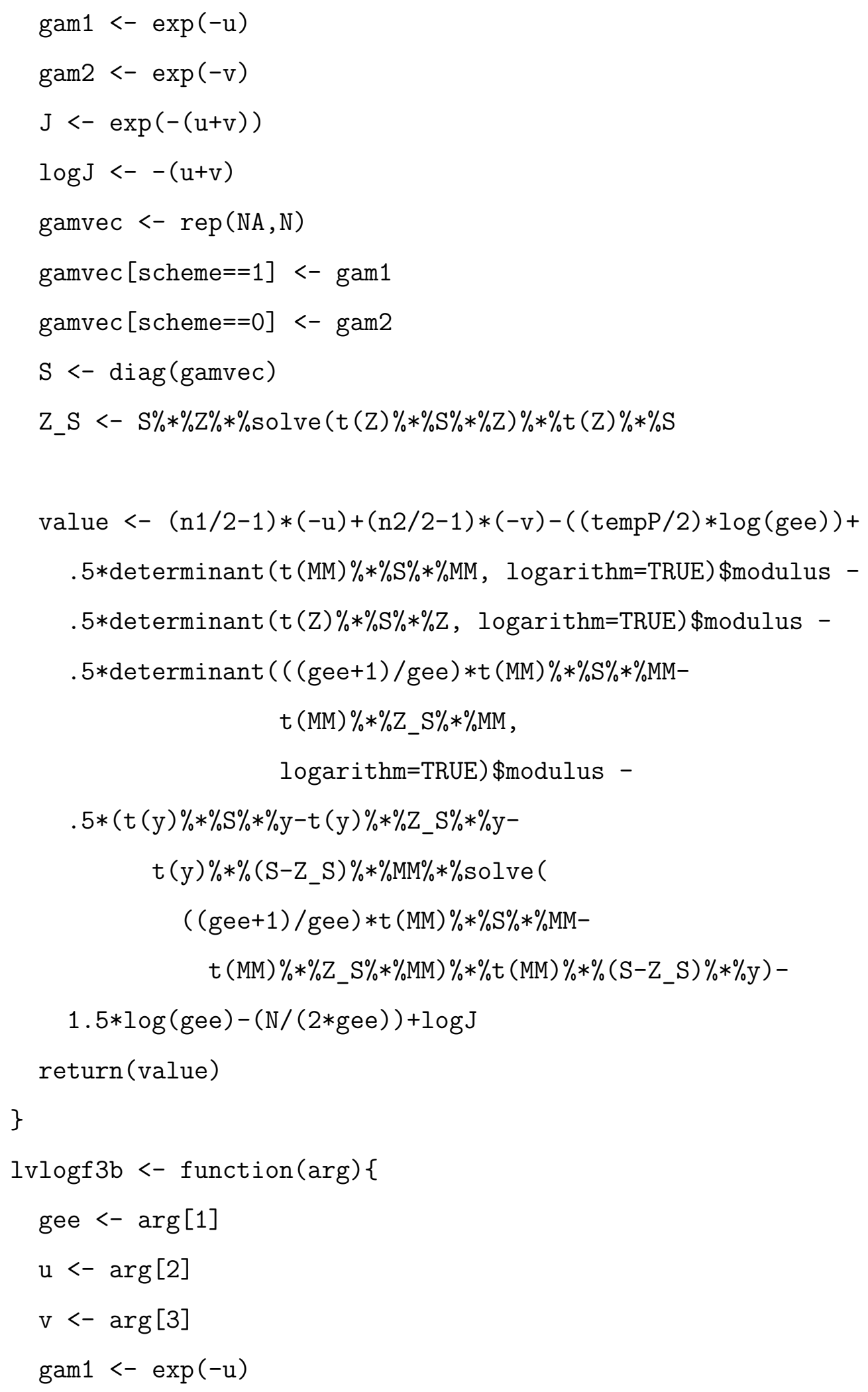




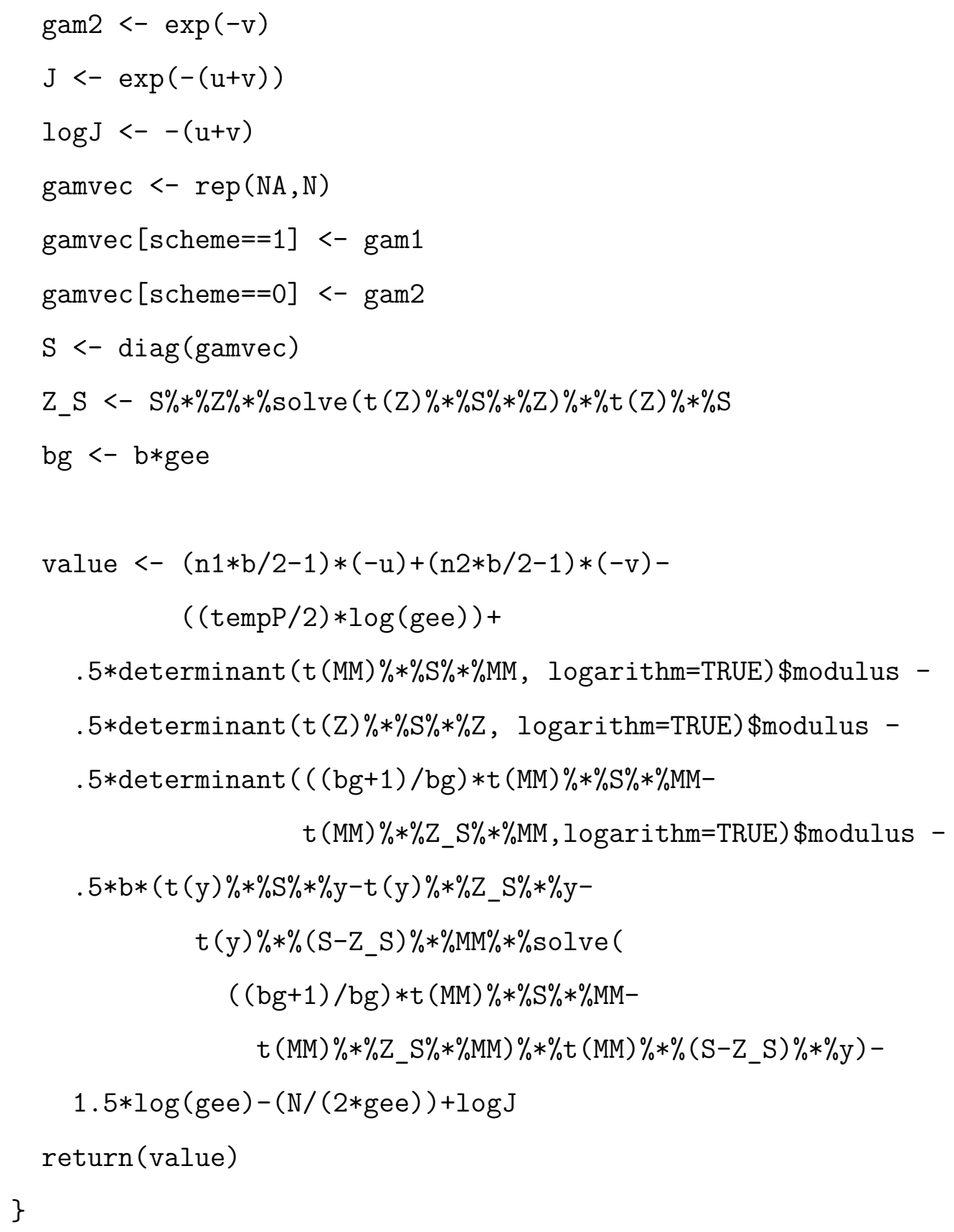




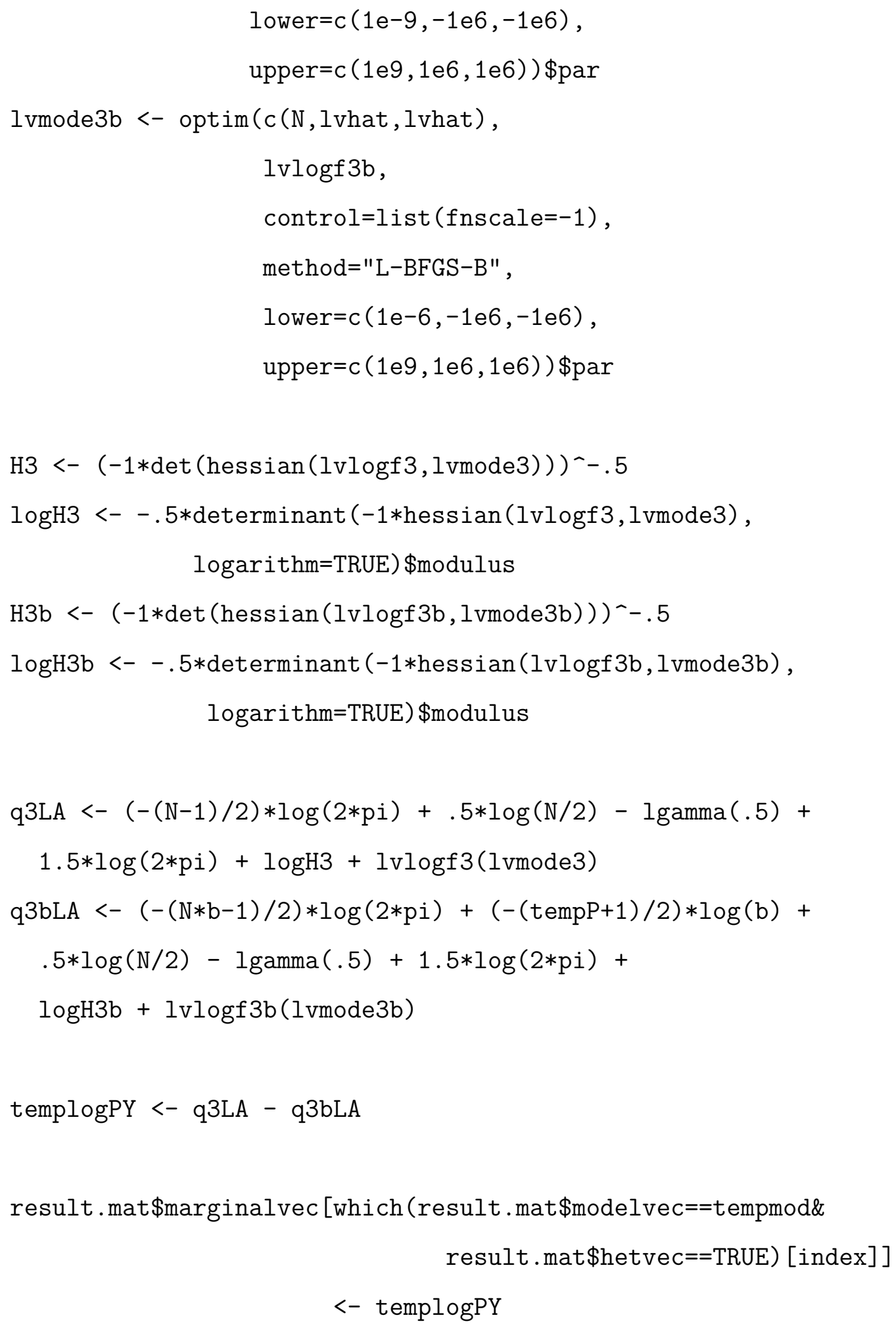




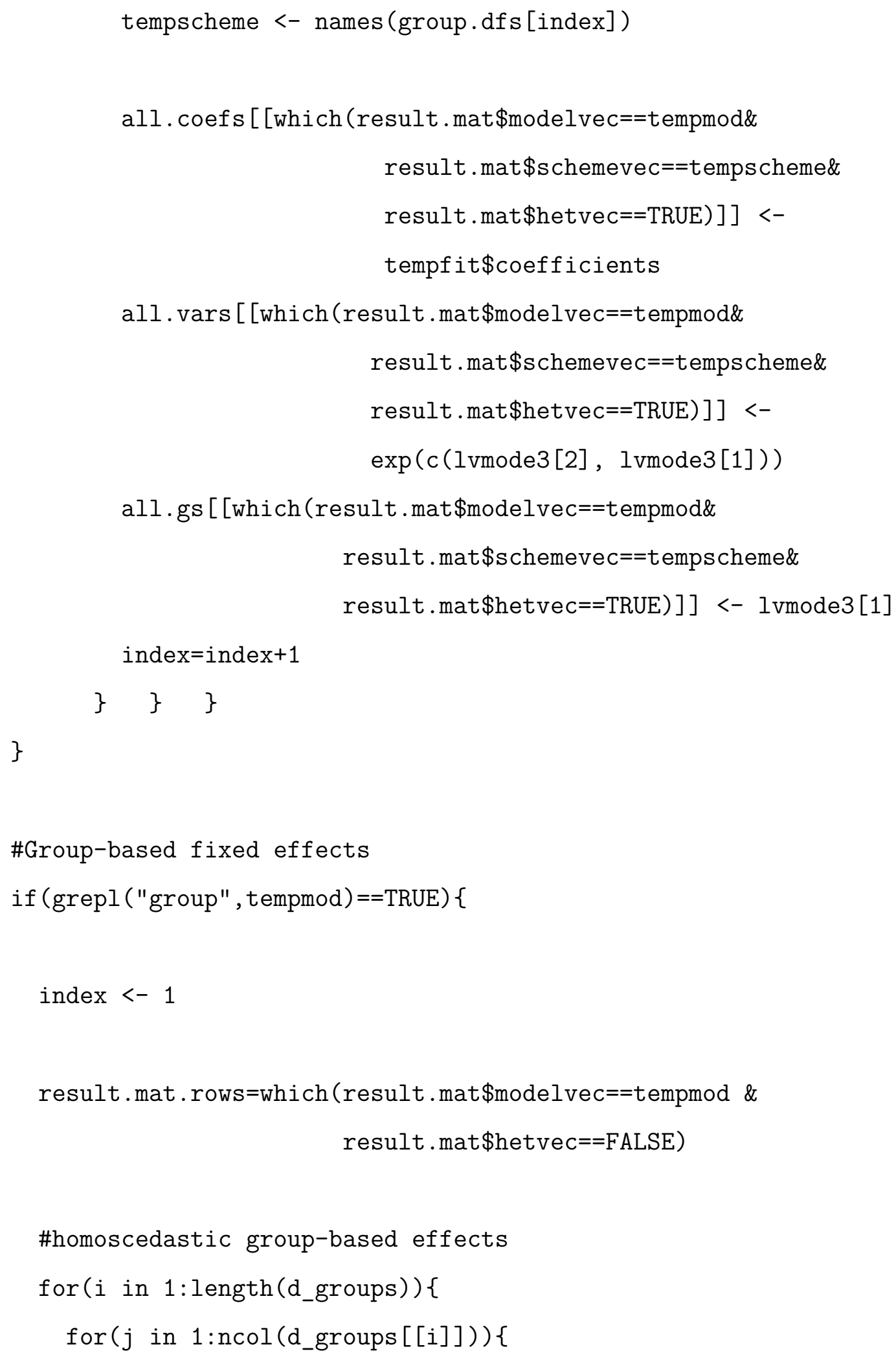


\{

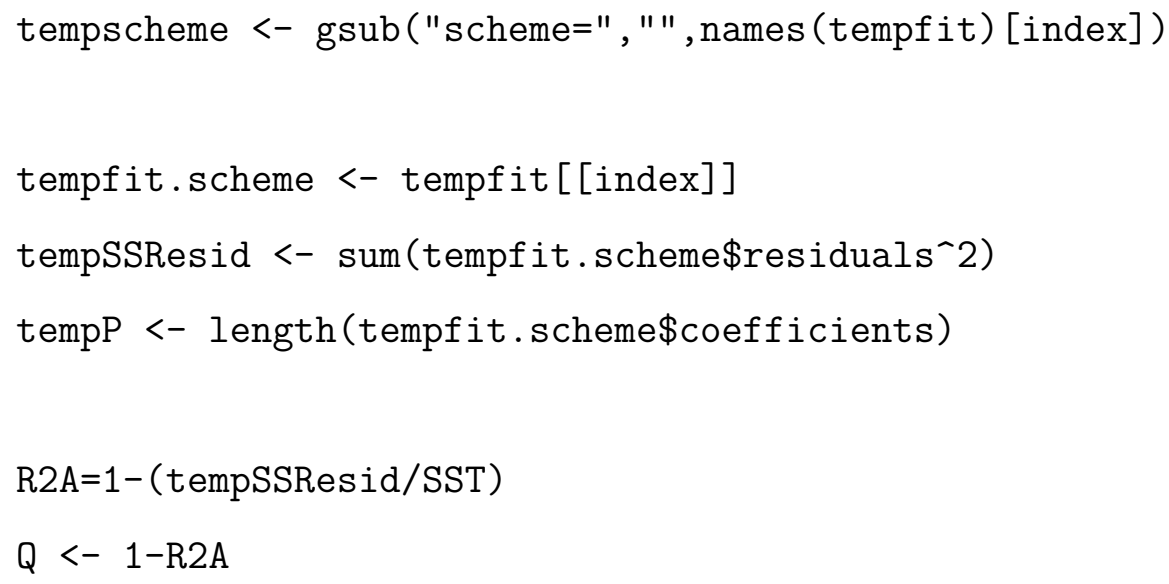




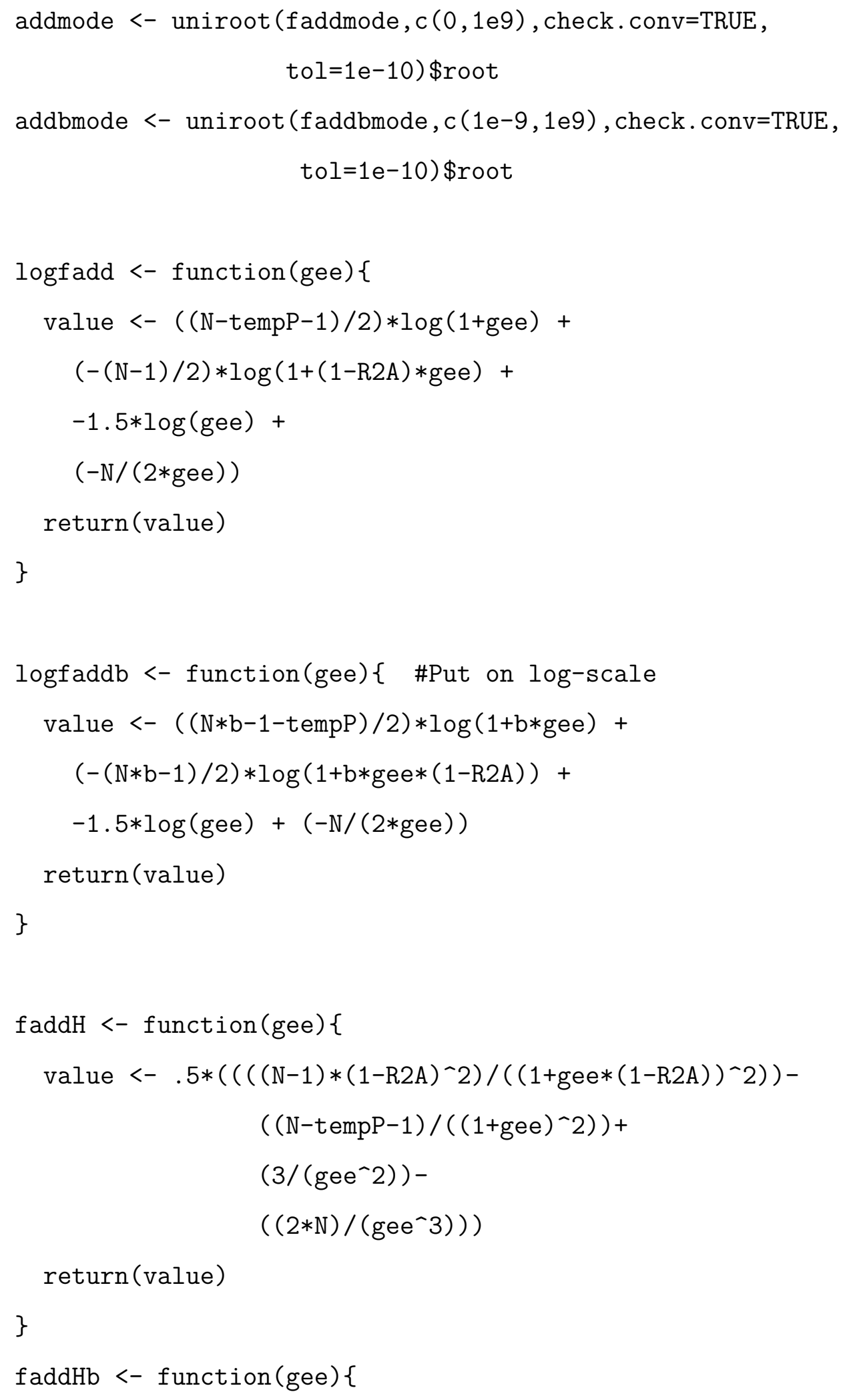




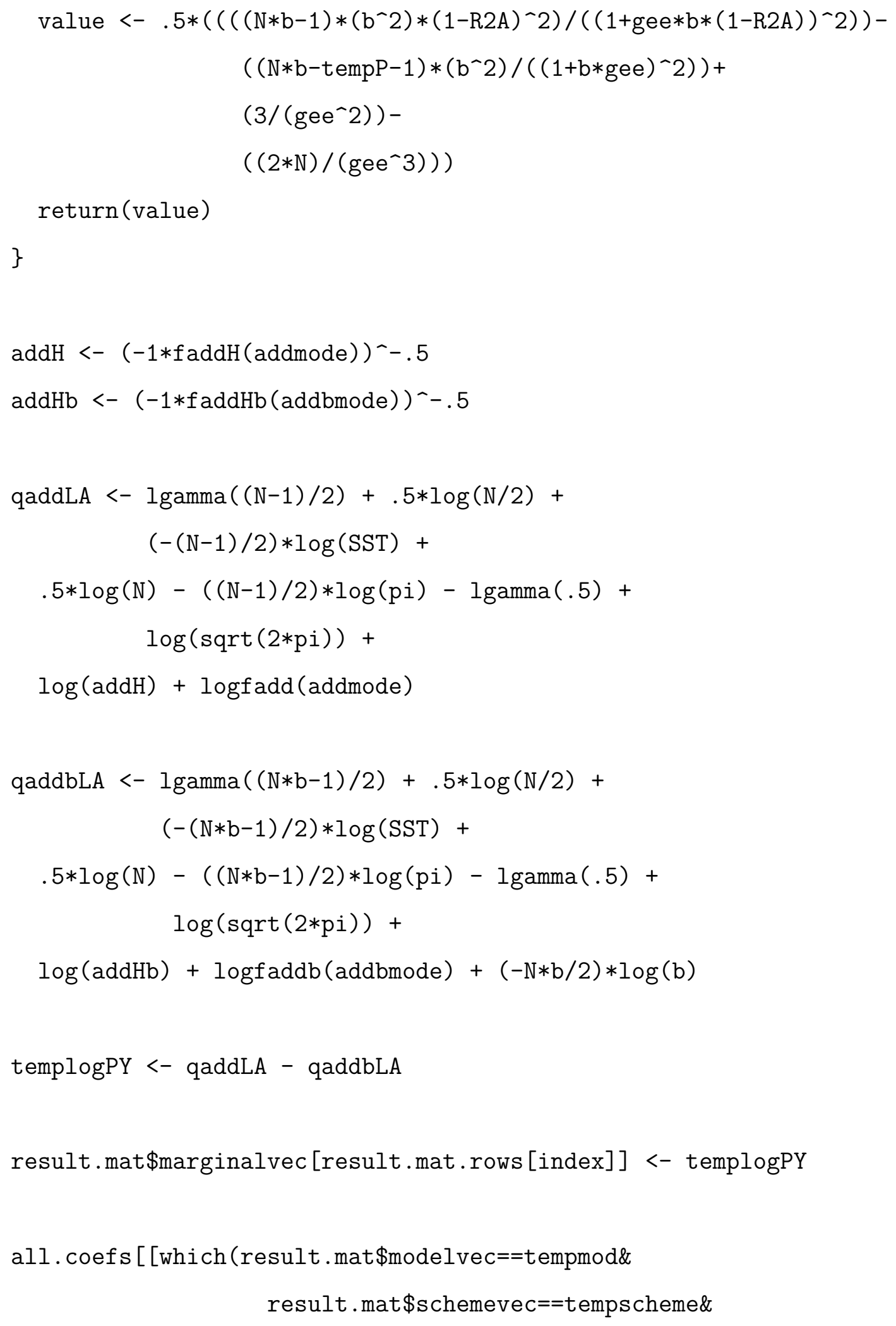




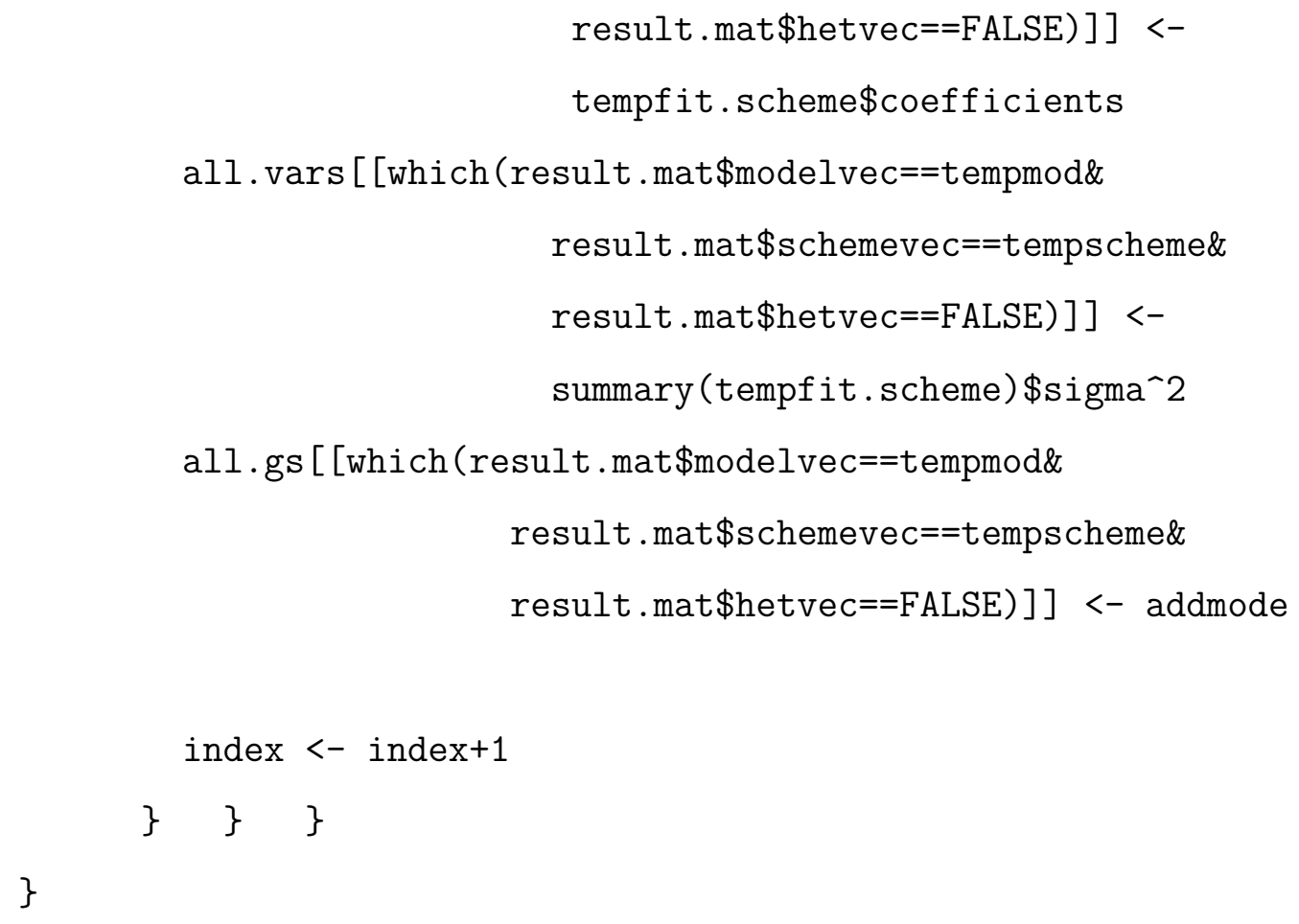




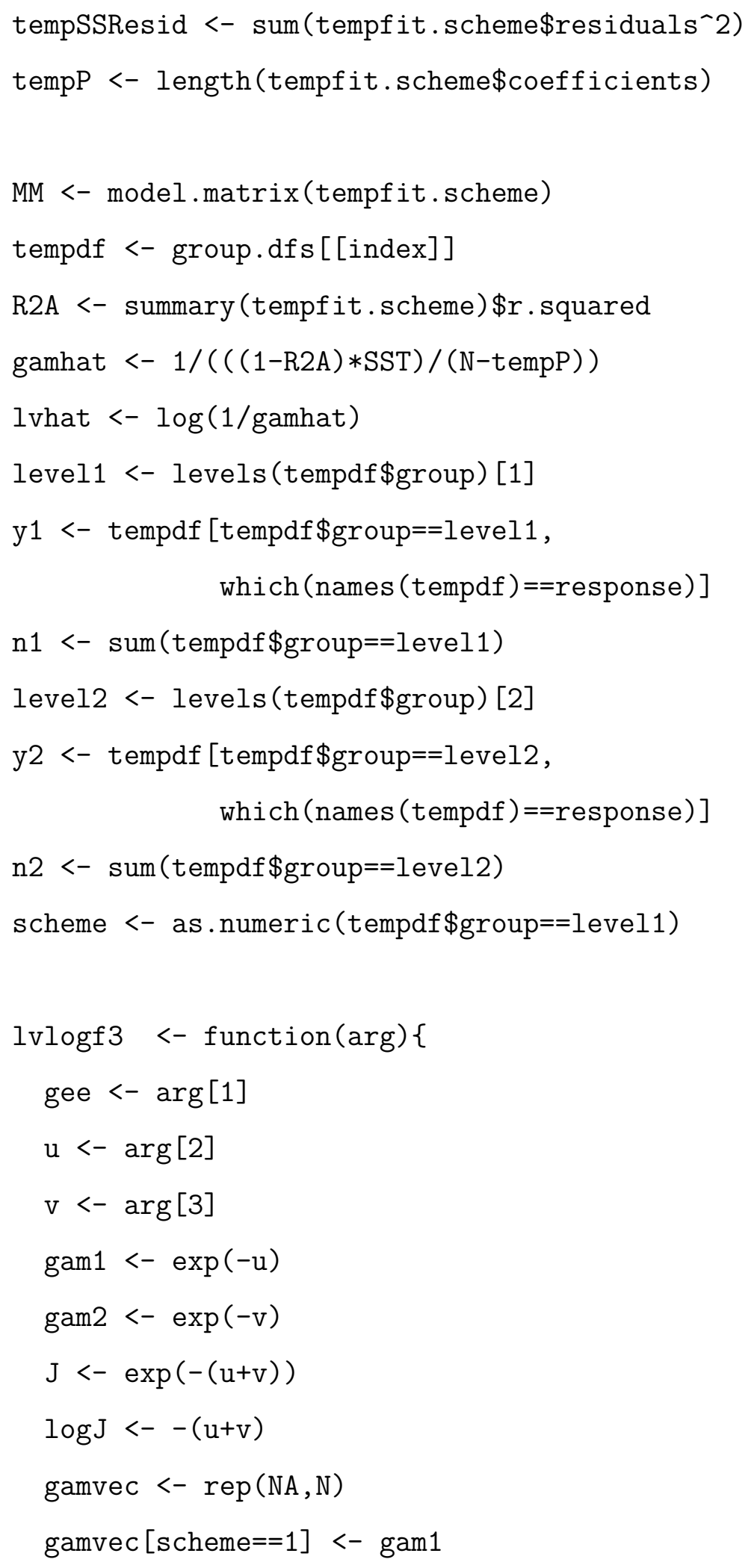




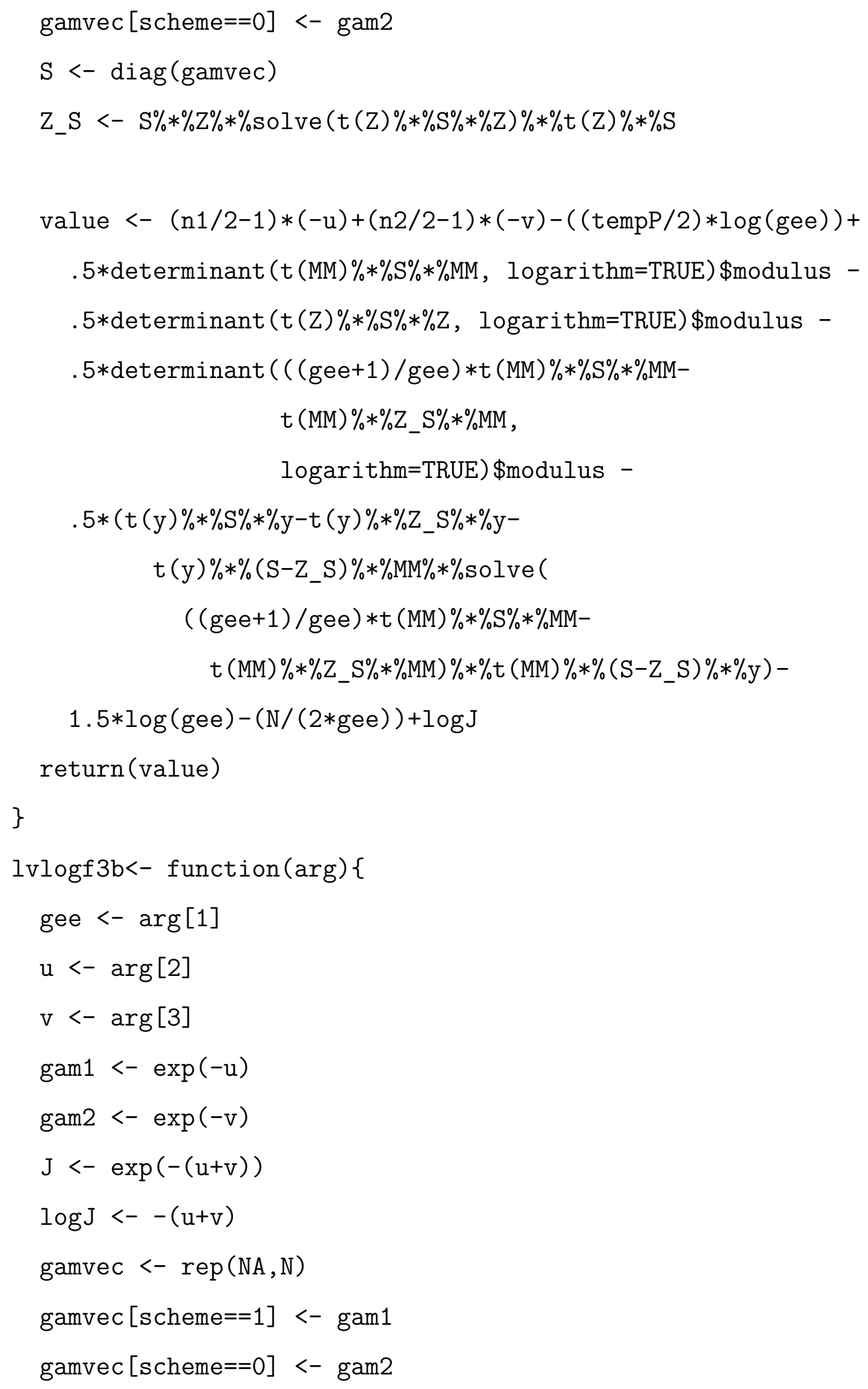




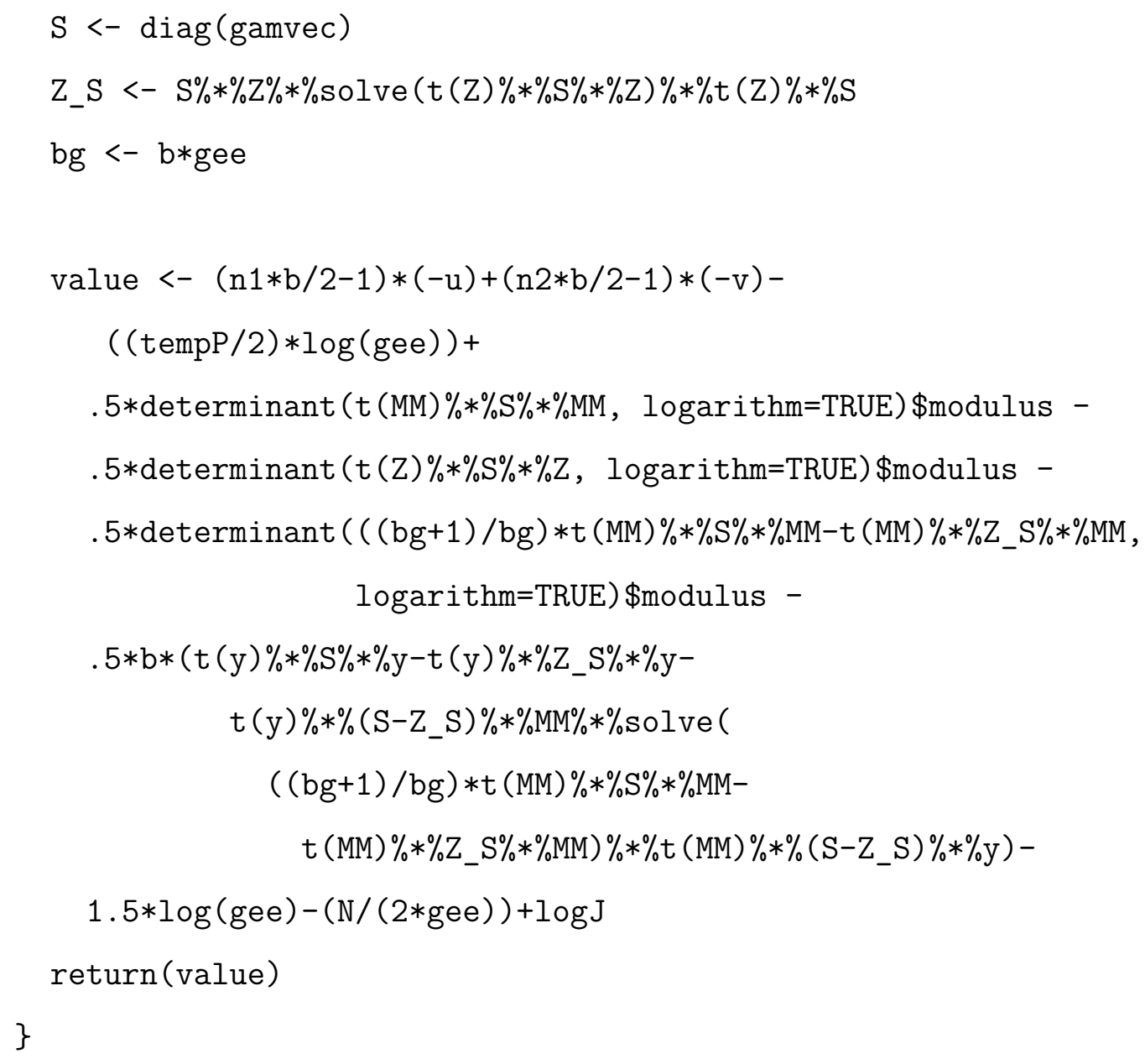




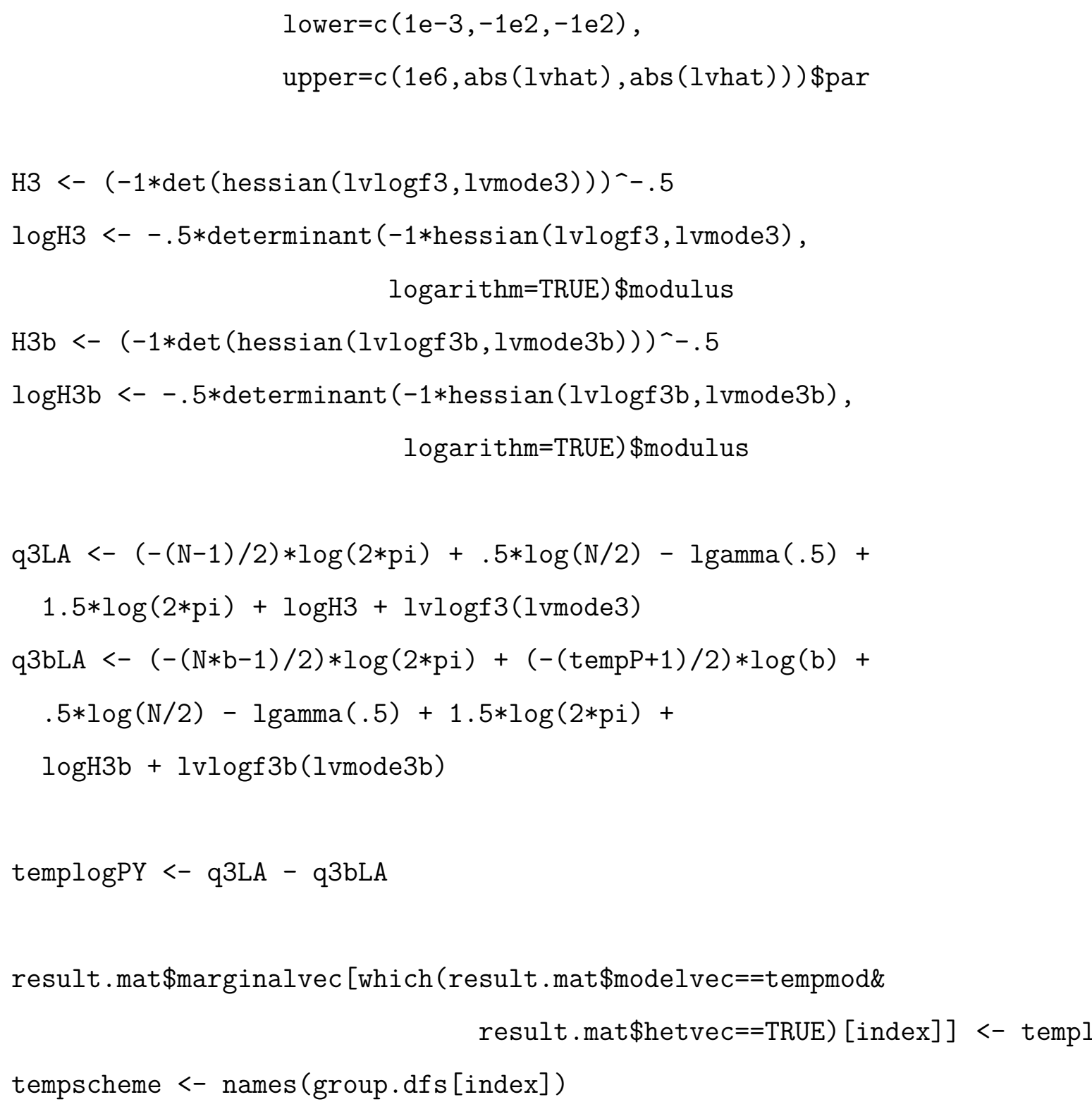

all . coefs [ [which (result . mat $\$$ modelvec==tempmod\& result . mat $\$$ schemevec $==$ tempscheme\& result . mat $\$$ hetvec==TRUE) ] ] <tempfit.scheme\$coefficients all $\cdot \operatorname{vars}[$ [which (result $\cdot$ mat $\$$ modelvec==tempmod\& result . mat $\$$ schemevec==temps cheme\& 


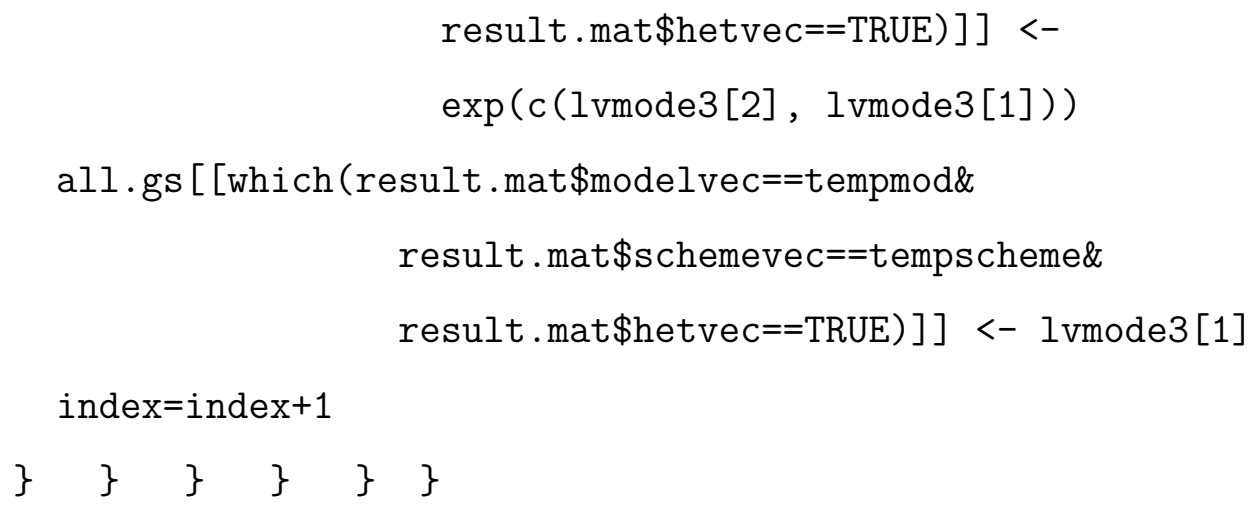




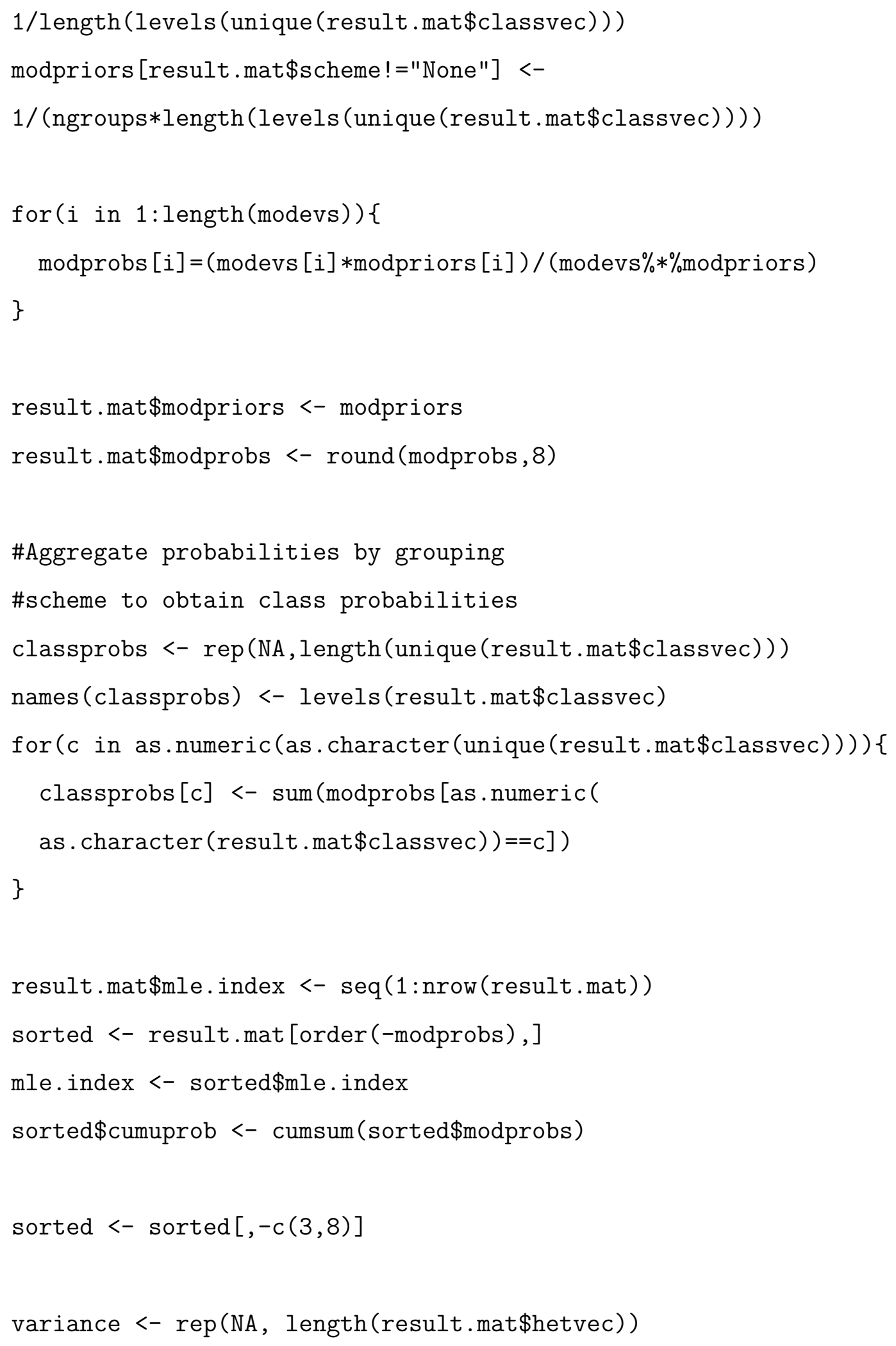




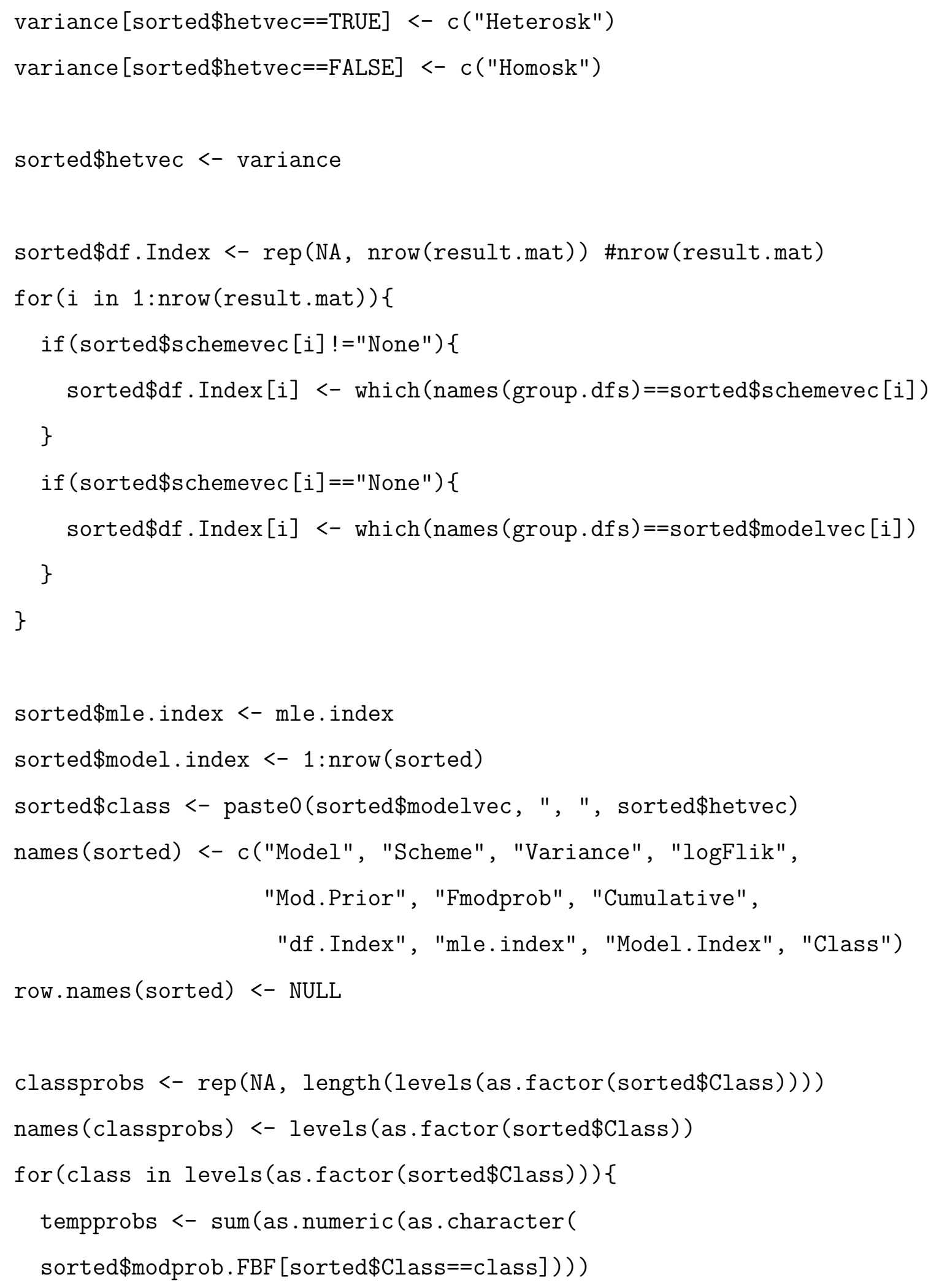




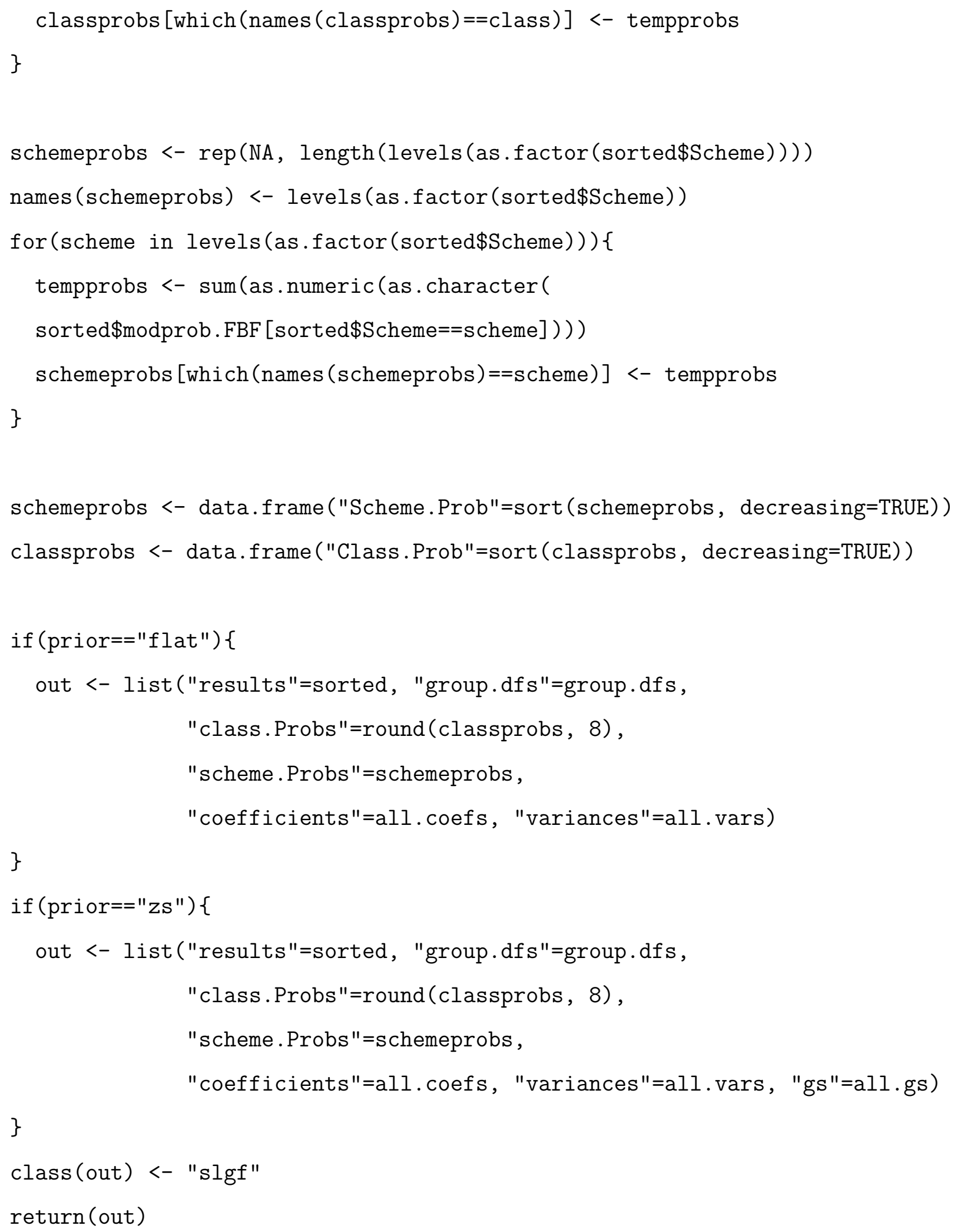


